Document downloaded from:

http://hdl.handle.net/10251/87837

This paper must be cited as:

Dhakshinamoorthy, A.; Opanasenko, M.; Cejka, J.; García Gómez, H. (2013). Metal organic frameworks as heterogeneous catalysts for the production of fine chemicals. Catalysis Science and Technology. 3(10):2509-2540. doi:10.1039/c3cy00350g

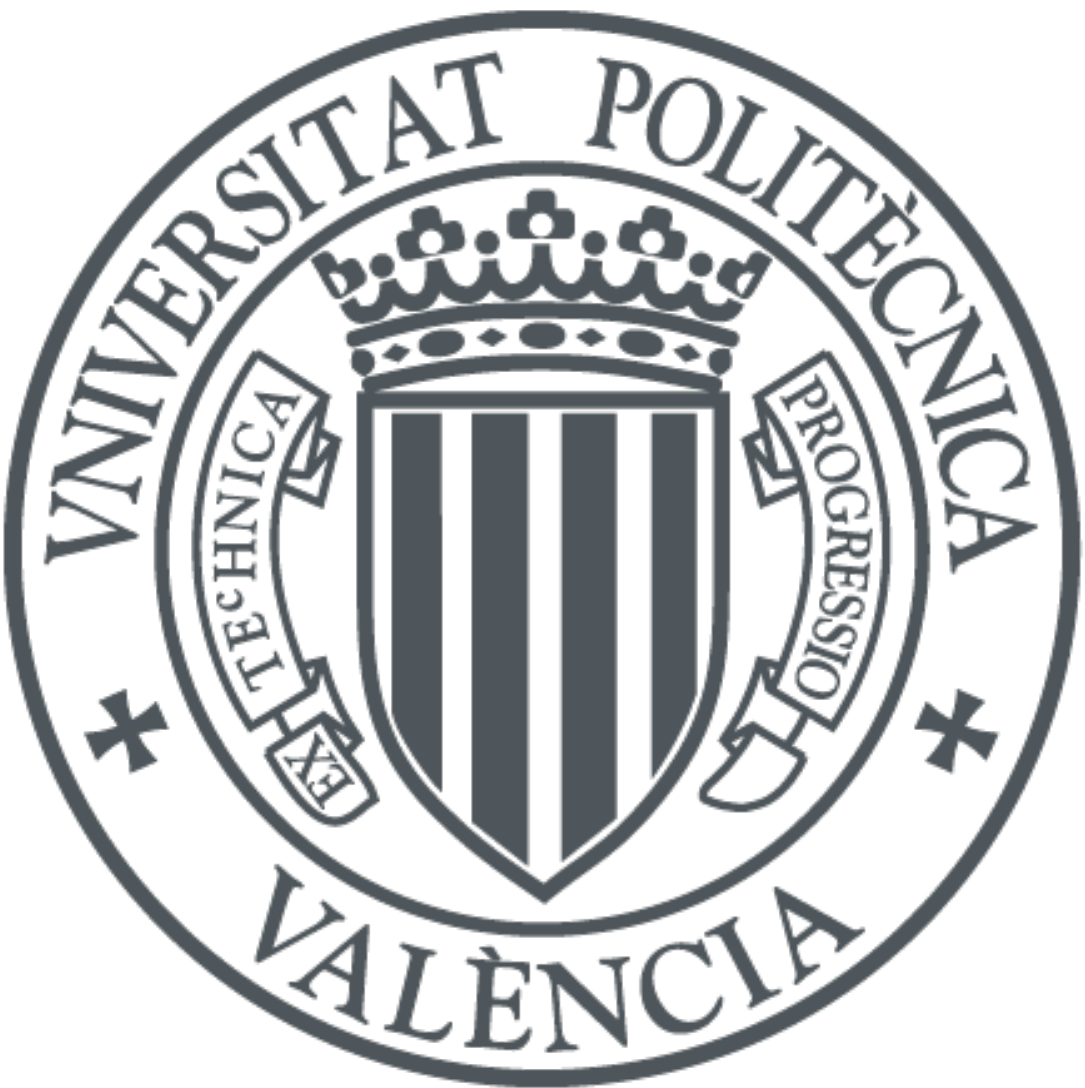

The final publication is available at

http://dx.doi.org/10.1039/c3cy00350g

Copyright Royal Society of Chemistry

Additional Information 


\section{Catalysis}

Science \& Technology

\section{Accepted Manuscript}

This article can be cited before page numbers have been issued, to do this please use: A. Dhakshinamoorthy, M. Opanasenko, J. Cejka and H. Garcia, Catal. Sci. Technol., 2013, DOI: 10.1039/C3CY00350G.

\section{Catalysis Science \&Technology}

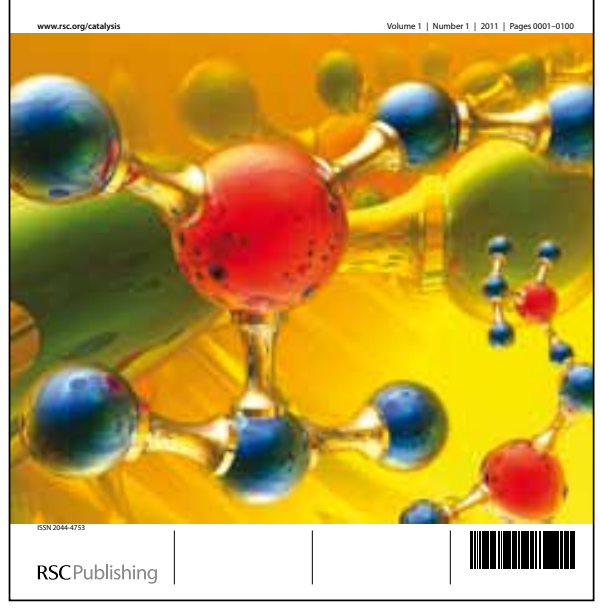

This is an Accepted Manuscript, which has been through the RSC Publishing peer review process and has been accepted for publication.

Accepted Manuscripts are published online shortly after acceptance, which is prior to technical editing, formatting and proof reading. This free service from RSC Publishing allows authors to make their results available to the community, in citable form, before publication of the edited article. This Accepted Manuscript will be replaced by the edited and formatted Advance Article as soon as this is available.

To cite this manuscript please use its permanent Digital Object Identifier (DOI ${ }^{\circledR}$, which is identical for all formats of publication.

More information about Accepted Manuscripts can be found in the

\section{Information for Authors.}

Please note that technical editing may introduce minor changes to the text and/or graphics contained in the manuscript submitted by the author(s) which may alter content, and that the standard Terms \& Conditions and the ethical guidelines that apply to the journal are still applicable. In no event shall the RSC be held responsible for any errors or omissions in these Accepted Manuscript manuscripts or any consequences arising from the use of any information contained in them. 


\title{
Metal organic frameworks as heterogeneous catalysts for the production of fine chemicals
}

\author{
Amarajothi Dhakshinamoorthy, ${ }^{*, a}$ Maksym Opanasenko, ${ }^{\mathrm{b}}$ Jiří Čejka*,b and Hermenegildo \\ Garcia*,c $^{*}$ \\ ${ }^{\mathrm{a}}$ School of Chemistry, Centre for Green Chemical Processes, Madurai Kamaraj University, \\ Tamil Nadu, India 625021. \\ ${ }^{\mathrm{b}}$ J. Heyrovsky Institute of Physical Chemistry, Academy of Sciences of Czech Republic, v.v.i. \\ Dolejškova 3, 18223 Prague 8 (Czech Republic). \\ ${ }^{\mathrm{c} I n s t i t u t o ~ d e ~ T e c n o l o g i ́ a ~ Q u i ́ m i c a ~ C S I V-U P V, ~ A v . ~ D e ~ l o s ~ N a r a n j o s ~ s / n, ~ 46022, ~ V a l e n c i a, ~ S p a i n . ~}$
}

\begin{abstract}
This review focuses on the use of metal organic frameworks (MOFs) as catalysts for the synthesis of fine chemicals. While petrochemistry is characterized by gas phase reaction, in which MOFs cannot compete with robust zeolites, MOFs are better suited for liquid phase reactions performed at moderate temperatures. These are the conditions typically employed for the production of fine chemicals characterized by being more complex and diverse molecules of low volatility, but with highly added value. For the preparation of this type of compounds, MOFs offer the advantage of wide open porosity in the nanometer scale and a large void volume. In the present review we have summarized the reports appeared up to early 2013 on the use of MOFs as catalysts in the liquid phase for the production of fine chemicals, primarily classified according to the type of active sites and the functional group formed in the reaction. Prospects for future development in this field are provided in the last section.
\end{abstract}




\section{List of abbreviations}

abr: anthracene-9,10-bis(5-resorcinol)

btp: 2,6-bis(N'-1,2,4-triazolyl)pyridine

bipy: 4,4'-bipyridine

bpe: 1,2-bis (4-pyridyl)-ethylene

bda: 2,2'-dihydroxy-1,1'-binaphthalene-5,5'-dicarboxylate

btc: 1,3,5-benzenetricarboxylate

btt: 1,3,5-benzene-tristetrazol-5-yl

btb: 4,4',4"-benzene-1,3,5-triyl-tribenzoate

bpa: 1,2-bis(4-pyridyl)ethane

btapa: 1,3,5-benzene tricarboxylic acid tris[N-(4-pyridyl)amide]

bdc $=1,4$-benzenedicarboxylate

bpdc: biphenyldicarboxylate

btec: 1,2,4,5-benzenetetracarboxylate

$\mathrm{H}_{2}$ bdpb: 1,4-bis[(3,5-dimethyl)pyrazol-4-yl]benzene

bpydc $=2,2$ '-bipyridine-5,5'-dicarboxylic acid

BINOL: 1,1'-bi-2-naphthol

DMF: dimethylformamide

$\mathrm{H}_{2}$ hfipbb: 4,4'-(hexafluoroisopropylidene) bis(benzoic acid)

Glu: glutarate

pdtc: pyridine-2,3,5,6-tetracarboxylate

pdai: 5,5'-((pyridine-3,5-dicarbonyl)bis-(azanediyl))diisophthalate tpdc: terphenyl-3,3'-dicarboxylate

PTA: phosphotungstic acid

POM: polyoxometallate

TBHP: tert-butylhydroperoxide

OCPP: metalloporphyrin octacarboxylates

MOF-199: $\left[\mathrm{Cu}_{2}(\mathrm{btc})_{4 / 3}\right]$

$\mathrm{C}_{4} \mathrm{H}_{4} \mathrm{O}_{4}$ : succinate

$\mathrm{PW}_{4}:\left[\mathrm{PW}_{4} \mathrm{O}_{24}\right]^{3-}$

$\mathrm{PW}_{12}:\left[\mathrm{PW}_{12} \mathrm{O}_{40}\right]^{3-}$

Pd-H tcpp: $_{4}$,10,15,20-tetra(carboxyphenyl)palladium-porphyrin 
IRMOF-3: Zn(2-atp) where 2-atp stands for 2-aminoterephthalic acid

MIL-101: $\left[\mathrm{Cr}_{3}(\mathrm{~F}, \mathrm{OH})-\left(\mathrm{H}_{2} \mathrm{O}\right)_{2} \mathrm{O}(\mathrm{bdc})_{3}\right]$

PCN-124: porous coordination network-124

SIM-1: $\mathrm{C}_{10} \mathrm{H}_{10} \mathrm{~N}_{4} \mathrm{O}_{2} \mathrm{Zn}$

TIF-1: tripodal imidazolate framework-1, $\left[\mathrm{Co}_{3} \mathrm{Cl}_{6}(1,3,5\right.$-tris(1H-imidazol-1-yl)-2,4,6-triethyl benzene $\left.)_{2}\right]$

ZIF-8: zeolitic imidazolate framework-8, $\left[\mathrm{Zn}\left(\mathrm{C}_{4} \mathrm{H}_{5} \mathrm{~N}_{2}\right)_{2}\right]$ where $\mathrm{C}_{4} \mathrm{H}_{5} \mathrm{~N}_{2}$ stands for 2-methylimidazolide

ZIF-9: zeolitic imidazolate framework-9, $\left[\mathrm{Zn}(2 \text {-benzimidazolide) })_{2}\right]$

UMCM-1: University of Michigan Crystalline Material-1, [ $\left.\mathrm{Zn}_{4} \mathrm{O}(\mathrm{bdc})(\mathrm{btb})_{4 / 3}\right]$

$\mathrm{ZJU}=$ Zhejiang University 


\section{Introduction}

Zeolites are industrial catalysts for many petrochemical processes carried out in the gas phase at very large scale. ${ }^{1,2}$ However, due to the limited available pore size, application of zeolites as solid catalysts in liquid phase reactions has been much more limited. ${ }^{2-4}$ In contrast to gas phase reactions where diffusion is much easier, zeolites as liquid phase catalysts encounter many problems arising from the restricted diffusion of substrates and products inside the micropores. ${ }^{5}$ Beside lower catalytic activity, restricted diffusion in a porous material is also connected with a high tendency of the catalyst to undergo deactivation by poisoning and pore blocking. ${ }^{6}$ Thus, while vapor phase reactions are suited for volatile simple hydrocarbons characteristic of petrochemistry and zeolites are ideal catalysts for these processes, these solids have many drawbacks for the production of fine chemicals. Fine chemicals are those compounds produced in lesser amounts than petrochemicals, but due to their structural complexity and functionality have much higher added value. ${ }^{7-19}$ They are generally more complex molecules and very often they exhibit low volatility due to the presence of functional groups. Transformation of fine chemicals is preferred in the liquid phase and under these conditions, zeolites can be far from being optimal catalysts, being a need for developing more efficient solid catalysts for this type of reaction.

Over the years, Chemical Industry has been using transition metal salts and specifically metal carboxylates as catalysts. ${ }^{12-22}$ For this reason, considering the composition of common metal organic frameworks containing transition metals and polycarboxylic aromatic compounds, ${ }^{23,} 24$ there was a logical interest in studying their catalytic properties, particularly compared to their non-porous salt analogues. MOFs can be defined as crystalline, porous solids, whose structure is defined by nodes of metal ions or clusters of metal ions held in the lattice by bi- and multipodal rigid organic linkers. ${ }^{23,25-30}$ The directionality of the metal-ligand coordination bonds is responsible for the creation of empty spaces and voids in the lattice, 
while the stability of the structure depends on the strength of these coordination forces that have intermediate energy between covalent bonds and weak intermolecular forces. Figure 1 summarizes the possible active sites present in MOFs that include metal nodes with free coordination sites, functional linkers or guests accommodated on the empty voids. Structural defects not expected in ideal frameworks are also increasingly recognized as potential active sites in catalysis.

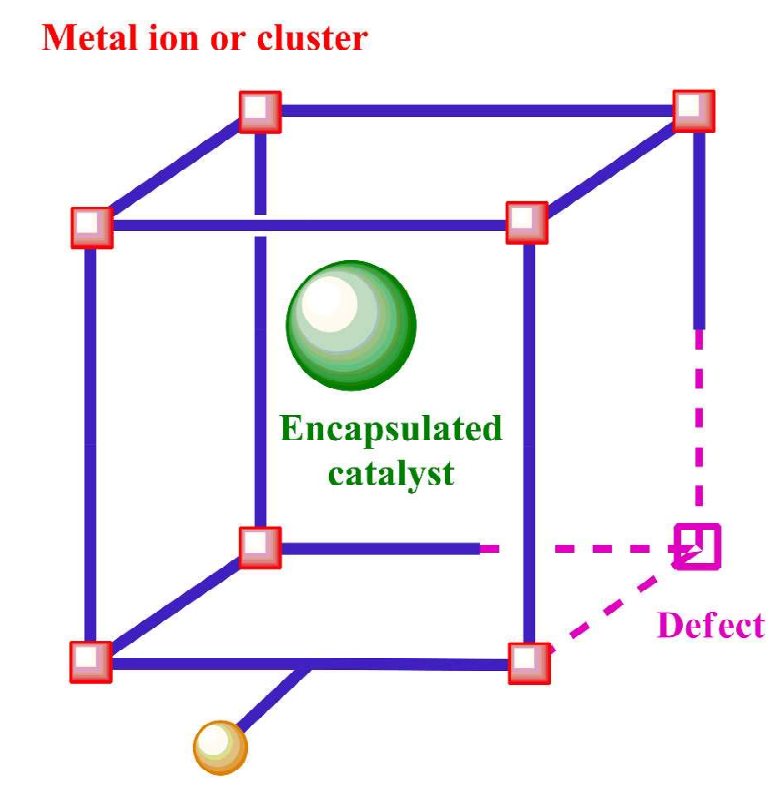

Functionalized linker

Figure 1. Different active sites available in MOFs responsible for catalysis

Stability to solvents, reactants and reaction conditions is one of the main limitations of the use of MOFs as solid catalysts, being necessary a careful survey of the MOF stability under each reaction conditions.

One advantage of MOFs is that these solids can be designed and their structure sometimes predicted by consideration of the directionality of the coordination bonds around the metal clusters and the geometry of the linker. ${ }^{29,}{ }^{31-33}$ There is also a large variety of organic linkers available and it has been reported MOFs with virtually all the transition metals. ${ }^{32,34}$ This flexibility in MOFs synthesis and design has been applied for the synthesis of catalysts ${ }^{26 \text {, }}$ 
${ }^{30}$ and materials for light harvesting and photocatalysis ${ }^{35}$ sharply contrasts with zeolites. ${ }^{36,37}$ Zeolites are generally obtained using of a structure directing agent, but it is still not possible to predict the structure of the resulting zeolite by analyzing the geometry and self assembly of the structure directing agent. In addition, the preparation of zeolites with pore sizes larger than 0.7 $\mathrm{nm}$ has been really difficult and there is a limitation in the dimensions of the pores available for zeolites. ${ }^{4,38,39}$ This is a key point in heterogeneous catalysis since diffusion inside the pores is strongly dependent on the relative dimensions of substrates, products and the pore dimensions. Particularly for liquid phase reactions, substrates and reagents can experience a strong diffusion limitation and accessing to the internal sites can be the key physical phenomenon determining the activity of a porous solid catalyst. Two-dimentional zeolites and mesoporous aluminosilicates can serve as an alternative to zeolites for liquid phase reactions. ${ }^{40,41}$

MOFs can be prepared with appropriate linkers that lead to wide open structures exhibiting much larger pore sizes, specific surface areas and pore volumes than conventional zeolites. $^{42}$ In fact, MOFs are the solids with the lowest framework density, meaning the minimum mass for a given unit cell volume, and this large porosity is extremely beneficial to minimize diffusion control, generally found in liquid phase due to the presence of solvent molecules. Nevertheless, it is clear that the internal diffusion of reagents and substrates through the pores and the possibility that the catalytic reaction is taking place inside the MOF particles should not be taken for granted and a complete catalytic study should focus specifically on this important issue. Participation of the internal sites in the catalysis can be supported by the lack of observation of size dependence effects on the reactivity of substrates with similar structure and different sizes and by the lack of influence of the crystallite size and external surface on the observed catalytic activity. ${ }^{43}$

Another important feature of MOFs is that their crystal structure and the periodicity and regularity of the active sites make these solids ideal candidates to apply the concept of "single 
site catalyst". Due to the random distribution of the first and second coordination sphere around a metal in metal oxides, zeolites and other materials, solid catalyst are characterized by having a distribution of catalytic sites, but not a single site. The aim in the field of solid as catalysts is to have a material in which all the sites have exactly the same structure that is the optimal to promote a given reaction. In this way, all the active sites will exhibit identical performance that would be the maximum possible activity. This situation can be, in principle be achieved in MOFs since the crystal lattice of the ideal structure would ensure that all the sites would have the same environment. ${ }^{44}$

All these factors including the possibility to synthesize MOFs with any desired metal, large metal content, large pore size and surface area, easy design and synthesis, make MOFs more advantageous than zeolites for liquid phase reactions, characteristic of fine chemistry. ${ }^{45}$ Figure 2 summarized the main features of MOFs as catalysts for the production of fine chemicals.

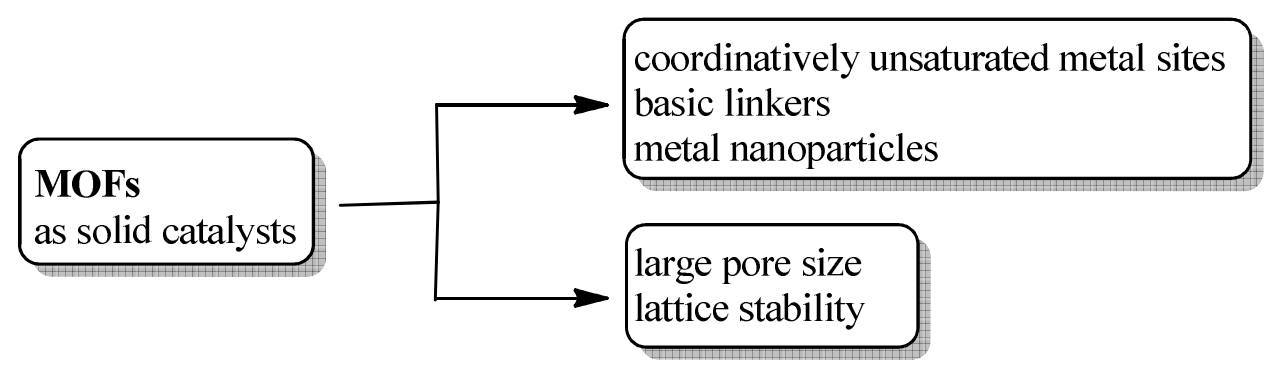

Figure 2. Main features of MOFs suitable as solid catalysts for the production of fine chemicals.

Also the reaction temperature range and experimental conditions for liquid phase reactions are more suited for MOFs considering their lower lattice stability compared to robust zeolites. However, at present valid comparisons of the activity of MOFs as catalysts with respect to that of zeolites or related microporous solids as well as related homogeneous metal salts are necessary to prove or disprove any potential structural advantage of MOF. These 
studies comparing the catalytic performance should serve to assess the benefits and drawbacks of MOFs as catalysts with respect to other soluble or heterogeneous alternatives.

The use of MOFs as heterogeneous catalysis in liquid phase is still under intense investigation, the target being to develop real industrial processes based on these materials. Considering the added value of fine chemicals and the typical reaction conditions for their production, MOFs appear as very promising catalysts in this sector. The present review describes the current state in the field of MOFs as heterogeneous catalysts for the production of fine chemicals, with the aim of stimulating further research in this field looking for the development of commercial applications of MOFs as industrial catalysts.

As we will comment (vide infra), additional advantage of MOFs with respect to other solid catalysts is the possibility to develop chiral solids for asymmetric induction. ${ }^{46-48}$ Asymmetric catalysis, in which the chirality of a catalyst promotes the selective formation of a desired enantiomer is very important in the production of many fine chemicals, particularly synthetic intermediates for the synthesis of drugs and molecules with biological and therapeutic activity. ${ }^{49-53}$ It has been very difficult to combine asymmetric catalysis with the use of solid catalysts, particularly with the use of zeolites, ${ }^{54-57}$ since asymmetric induction arises from subtle differences in the stability of the transition states leading to each enantiomer. In this context, the use of a single enantiomer of a chiral linker in MOF synthesis appears as a general methodology to achieve enantioselectivity in heterogeneous catalysis applied to the synthesis of chiral compounds as we will describe below. ${ }^{46}$ Recently, design, synthesis, and structural characteristics of chiral MOFs as well as their catalytic activities in various asymmetric transformations have been reported. ${ }^{58,} 59$ In the present review emphasis has been made describing the examples in which the use of chiral MOFs has been applied to the synthesis of fine chemicals. 
Last but not least, MOFs offer also many possibilities to develop tandem reactions, in which two or more reactions take place consecutively promoted by a single catalyst. Tandem reactions may require the presence of different catalytic centers that should be placed in adequate positions of the framework in such a way that they do not interfere negatively. ${ }^{60-62}$ The topology of MOFs with empty spaces, nodal positions and linkers provide at least three different opportunities to locate different centers in the same material. One common example of these tandem processes is aldol condensation followed by hydrogenation that can be promoted by acid sites and noble metal nanoparticles, respectively. ${ }^{63,}{ }^{64}$ Tandem reactions, in which several individual transformations have been coupled in one process, are one possibility for process intensification that is largely pursued in industry due to the economical advantages.

The present review focuses on the use of MOFs as solid catalysts for the production of fine chemicals. The reader is referred to the already existing general reviews on MOFs as heterogeneous catalysts for a comprehensive overview of application of MOFs in catalysis. ${ }^{26,53}$, ${ }^{65-67}$ The field of the catalytic activity of MOFs incorporating as metal nanoparticles or metal complexes has been recently reviewed. ${ }^{66,68}$ To minimize overlapping with existing recent reviews, we have also not considered those reports in which MOFs have been used as solid matrices to embed metal or metal oxides nanoparticles that are typically used as catalysts for CC couplings, oxidation and hydrogenation, cyanosilylation among other reactions. ${ }^{66,68}$ Herein, we will concentrate only on those transformations occurring in the liquid phase leading to products of interest as synthetic intermediates or compounds of commercial value in the absence of metal nanoparticles. This review is primarily organized in three main sections (acid/base, oxidation and reduction) according to the nature of the sites that promote the reaction. These three sections have been subdivided according to the functional groups of the resulting compounds. A final section summarizes the main conclusions and provides our view on future developments in the field. 


\section{Fine chemicals by acid-base catalysis over MOFs}

Table 1 lists those MOFs that have been used as catalysts for the preparation of fine chemicals with indication of the reaction type and the corresponding reference. The processes have in common the required acid or basic property of the catalyst. In the next paragraphs, we have classified these reports according to the type of product that is being formed.

Table 1. List of the MOFs that have been used as catalysts for the preparation of fine chemicals and the corresponding reactions.

\begin{tabular}{|c|c|c|}
\hline Reaction & MOF formula & Ref. \\
\hline $\begin{array}{l}\text { Alkyl/alkynylzinc addition } \\
\text { to aldehydes }\end{array}$ & $\begin{array}{l}(\mathrm{ZrL}) \cdot 4-5 \mathrm{H}_{2} \mathrm{O} / \mathrm{Ti}(\mathrm{OiPr})_{4} \\
{\left[\mathrm{Cd}_{3} \mathrm{Cl}_{6} \mathrm{~L}_{3}\right] \cdot 4 \mathrm{DMF} \cdot 6 \mathrm{MeOH} \cdot 3 \mathrm{H}_{2} \mathrm{O} / \mathrm{Ti}(\mathrm{OiPr})_{4}} \\
{\left[\mathrm{Cd}_{3} \mathrm{~L}_{4}\left(\mathrm{NO}_{3}\right)_{6}\right] \cdot 7 \mathrm{MeOH} \cdot 5 \mathrm{H}_{2} \mathrm{O} / \mathrm{Ti}(\mathrm{OiPr})_{4}} \\
{\left[\mathrm{Zn}_{2}(\mathrm{~L})(\mathrm{DMF})\left(\mathrm{H}_{2} \mathrm{O}\right)\right] \cdot 2 \mathrm{EtOH} \cdot 4.3 \mathrm{DMF} \cdot 4 \mathrm{H}_{2} \mathrm{O} / \mathrm{Ti}(\mathrm{O}} \\
\mathrm{iPr})_{4} \\
{\left[\mathrm{LCu}_{2}(\text { solvent })_{2}\right] / \mathrm{Ti}(\mathrm{OiPr})_{4}}\end{array}$ & $\begin{array}{l}69 \\
70 \\
71 \\
72\end{array}$ \\
\hline $\begin{array}{l}\text { Addition of Grignard } \\
\text { reagent to oxocompounds }\end{array}$ & {$\left[\mathrm{Cu}_{2} \mathrm{~L}_{2} \mathrm{Cl}_{2}\right] \cdot \mathrm{H}_{2} \mathrm{O}$} & 27 \\
\hline Alcoholysis of epoxides & $\begin{array}{l}\mathrm{Cu}-\mathrm{MOF} \\
{\left[\mathrm{Cu}\left(\mathrm{ClO}_{4}\right)_{2}(\mathrm{btp})_{2}\right] \text { and }\left[\mathrm{Cu}(\mathrm{btp})\left(\mathrm{H}_{2} \mathrm{O}\right)_{4}\right]\left(\mathrm{SO}_{4}\right) \cdot 2 \mathrm{H}_{2} \mathrm{O}} \\
{\left[\mathrm{Cu}\left(\mathrm{H}_{2} \mathrm{O}\right)_{2}-(\mathrm{btp})_{2}-\mathrm{Cu}\left(\mathrm{NO}_{3}\right)_{2}\right]\left(\mathrm{PF}_{6}\right)_{2}} \\
{\left[\mathrm{Cu}(\text { bipy })\left(\mathrm{H}_{2} \mathrm{O}\right)_{2}\left(\mathrm{BF}_{4}\right)_{2}(\text { bipy })\right]} \\
\mathrm{Cu}-\mathrm{MOF} \\
\text { Ni(L-aspartate)bipy } 0.5 \text { and } \mathrm{Cu}(\mathrm{L} \text {-aspartate }) \text { bpe }_{0.5}\end{array}$ & $\begin{array}{l}74 \\
75 \\
76 \\
77 \\
78 \\
79\end{array}$ \\
\hline Aminolysis of epoxides & $\begin{array}{l}{\left[\mathrm{Cu}_{2}(\text { bda })_{2}\left(\mathrm{H}_{2} \mathrm{O}\right)_{2}\right] \cdot \mathrm{MeOH} \cdot 2 \mathrm{H}_{2} \mathrm{O}} \\
{\left[\mathrm{Cu}(\text { bipy })\left(\mathrm{H}_{2} \mathrm{O}\right)_{2}\left(\mathrm{BF}_{4}\right)_{2}(\text { bipy })\right]}\end{array}$ & $\begin{array}{l}80 \\
77\end{array}$ \\
\hline Nitroaldol (Henry) reaction & $\begin{array}{l}{\left[\mathrm{Cu}_{3}(\mathrm{pdtc}) \mathrm{L}_{2}\left(\mathrm{H}_{2} \mathrm{O}\right)_{3}\right] \cdot 2 \mathrm{DMF} \cdot 10 \mathrm{H}_{2} \mathrm{O}} \\
{[\mathrm{Zn}(\mathrm{tpdc})] \cdot \mathrm{DMF} \cdot 2 \mathrm{H}_{2} \mathrm{O}}\end{array}$ & $\begin{array}{l}81 \\
82\end{array}$ \\
\hline $\begin{array}{l}\text { Dehydration of methanol to } \\
\text { dimethyl ether }\end{array}$ & MIL-101(Cr)/PTA & 83 \\
\hline
\end{tabular}




\begin{tabular}{|c|c|c|}
\hline $\begin{array}{l}\text { Conjugate addition of } \\
\text { alcohols to } \alpha, \beta \text {-unsaturated } \\
\text { ketones }\end{array}$ & TIF-1 & 84 \\
\hline Acetalization of aldehydes & {$\left[\mathrm{Yb}\left(\mathrm{C}_{4} \mathrm{H}_{4} \mathrm{O}_{4}\right)_{1.5}\right]$} & 85 \\
\hline Ketalization of ketones & {$\left[\mathrm{LCuPF}_{6} \cdot \mathrm{xH}_{2} \mathrm{O}\right]$} & 86 \\
\hline Diels-Alder cycloaddition & $\begin{array}{l}{\left[\mathrm{Zr}_{2}(\mathrm{OR})_{4}(\mathrm{abr})\right],\left[\mathrm{Ti}_{2}(\mathrm{OR})_{2} \mathrm{Cl}_{2}(\mathrm{abr})\right], \quad\left[\mathrm{Al}_{2} \mathrm{Cl}_{2}(\mathrm{abr})\right] \text {, }} \\
{\left[\mathrm{Al}_{2}(\mathrm{OR})_{2}(\mathrm{abr})\right]} \\
\text { set of MOFs }\end{array}$ & $\begin{array}{l}87-89 \\
90\end{array}$ \\
\hline Meinwald rearrangement & $\begin{array}{l}\mathrm{MIL}-101(\mathrm{Al})-\mathrm{NH}_{2} \\
\mathrm{Fe}(\mathrm{btc})\end{array}$ & $\begin{array}{l}91 \\
92\end{array}$ \\
\hline Pechmann condensation & $\mathrm{Cu}_{3}(\mathrm{btc})_{2}$ and $\mathrm{Fe}(\mathrm{btc})$ & 93 \\
\hline Friedel-Crafts acylation & $\begin{array}{l}\mathrm{Cu}_{3}(\mathrm{btc})_{2}, \mathrm{MIL}-100(\mathrm{Fe} / \mathrm{Cr}) \\
\text { IRMOF-8 } \\
\text { ZIF-8 }\end{array}$ & $\begin{array}{l}94 \\
95 \\
96\end{array}$ \\
\hline $\begin{array}{l}\text { Dehydration } \\
\text { fructose/glucose }\end{array}$ & MIL-101(Cr)/PTA & 97 \\
\hline Aldol reaction & $\begin{array}{l}{\left[\mathrm{Mn}_{3}\left\{\left(\mathrm{Mn}_{4} \mathrm{Cl}\right)_{3}(\mathrm{btt})_{8}\left(\mathrm{CH}_{3} \mathrm{OH}\right)_{10}\right\}_{2}\right]} \\
\text { Cd-MOF } \\
\text { modified MIL-101 } \\
\text { Zn-IRMOF } \\
\text { metalated UMCM-1-NH } \\
{\left[\mathrm{Zn}_{3}(\mathrm{btb})_{2}\right], \text { MIL-101 }}\end{array}$ & $\begin{array}{l}98 \\
99 \\
100 \\
101 \\
102 \\
103\end{array}$ \\
\hline Transesterification & $\begin{array}{l}{\left[\left\{\mathrm{Zn}_{3}\left(\mu_{3}-\mathrm{O}\right)(\mathrm{L})_{6}\right\} \cdot 2 \mathrm{H}_{3} \mathrm{O} \cdot 12 \mathrm{H}_{2} \mathrm{O}\right]} \\
\mathrm{ZIF}-8 \\
{\left[\left\{\mathrm{Cu}_{2}(\mathrm{Glu})_{2}(\mu-\mathrm{bpa})\right\} \cdot\left(\mathrm{CH}_{3} \mathrm{CN}\right)\right]}\end{array}$ & $\begin{array}{l}104 \\
105 \\
106\end{array}$ \\
\hline Carbonyl-ene reaction & $\begin{array}{l}\text { Ti complexes of BINOL derivatives } \\
{\left[\mathrm{Cu}_{2}(\mathrm{~L})_{2}\left(\mathrm{H}_{2} \mathrm{O}\right)_{2}\right]}\end{array}$ & $\begin{array}{c}107,108 \\
109\end{array}$ \\
\hline Knoevenagel condensation & $\begin{array}{l}\text { MIL-101(Cr)-NH } 2 \\
\text { MIL-101(Al/Fe)-NH } \\
\text { ZIF-9 } \\
\text { PCN-124 }\end{array}$ & $\begin{array}{c}110,111 \\
112-114 \\
115 \\
116\end{array}$ \\
\hline
\end{tabular}




\begin{tabular}{|l|l|c|}
\hline & $\begin{array}{l}\mathrm{IRMOF}-3 \\
\left.\left[\mathrm{Cd}(4-\mathrm{btapa})_{2}\left(\mathrm{NO}_{3}\right)_{2}\right] \cdot 6 \mathrm{H}_{2} \mathrm{O} \cdot 2 \mathrm{DMF}\right] \\
\mathrm{Cu}_{3}(\mathrm{btc})_{2}, \mathrm{Fe}(\mathrm{btc})\end{array}$ & 118 \\
\hline N-alkylation & $\mathrm{Al}_{2}(\mathrm{bdc})_{3}, \mathrm{Cu}_{3}(\mathrm{btc})_{2}, \mathrm{Fe}(\mathrm{btc})$ & 119 \\
\hline Aza-Michael reaction & $\mathrm{MOF}-199$ & 120 \\
\hline Beckmann rearrangement & $\mathrm{Cu}_{3}(\mathrm{btc})_{2}, \mathrm{Fe}(\mathrm{btc})$ & 122 \\
\hline Biginelli reaction & {$\left[\mathrm{Cu}_{2} \mathrm{~L}_{2} \mathrm{Cl}{ }_{2}\right] \cdot \mathrm{H}_{2} \mathrm{O}$} & 27 \\
\hline $\begin{array}{l}\text { Rearrangement of } \alpha-\text { pinene } \\
\text { oxide }\end{array}$ & $\mathrm{Fe}(\mathrm{btc}), \mathrm{MIL}-88 \mathrm{~B}, \mathrm{MIL}-88 \mathrm{C}, \mathrm{MIL}-100, \mathrm{MIL}-127$ & 92 \\
\hline Cyclization of citronellal & $\mathrm{Cu}_{3}(\mathrm{btc})_{2}$ & 123 \\
\hline Prins condensation & $\begin{array}{l}\mathrm{Cu}_{3}(\mathrm{btc})_{2}, \mathrm{Fe}(\mathrm{btc}), \mathrm{MIL}-100(\mathrm{Fe} / \mathrm{Cr}), \text { ZIF-8, MIL- } \\
53(\mathrm{Al})\end{array}$ & 124 \\
\hline
\end{tabular}

\section{Synthesis of alcohols}

\section{Addition of diethylzinc or Grignard reagent to aldehydes}

Alcohols have many scientific, medical and industrial uses. ${ }^{125}$ Formation of secondary or tertiary alcohols by addition of organometallic compounds to carbonyl groups are one of the most studied reaction using MOFs as catalysis. Diethylzinc $\left(\mathrm{ZnEt}_{2}\right)$ has been one of the preferred reagents for this nucleophilic addition. ${ }^{126}$ Due to the lower reactivity of $\mathrm{ZnEt}_{2}$ compared to Grignard and other organometallic reagents, a catalyst is needed to promote the addition. $\mathrm{Ti}^{4+}$ complexes are widely used as homogeneous Lewis acid catalysts for the addition of $\mathrm{ZnEt}_{2}$ to aldehydes, including the enantioselective version of this process using chiral $\mathrm{Ti}$ catalysts. $^{127}$

In this context, Lin and coworkers studied different MOFs catalysts for $\mathrm{ZnEt}_{2}$ addition to aromatic aldehydes. ${ }^{69}$ They reported the synthesis of Ti-containing chiral porous MOF based on BINOL-derived $\mathrm{Zr}$ phosphonates active in asymmetric $\mathrm{ZnEt}_{2}$ addition to aromatic aldehydes with high conversion and enantiomeric excess (ee) value up to $72 \%{ }^{69}$ 
Later, the same group reported the synthesis of a Cd-containing chiral porous MOF by using chiral bridging ligands - (R)-6,6'-dichloro-2,2'-dihydroxy-1,1'-binaphthyl-bipy. ${ }^{70}$ After postsynthetic treatment with $\mathrm{Ti}\left(\mathrm{O}^{\mathrm{i}} \mathrm{Pr}\right)_{4}$, a catalytically active Lewis acid complex was formed. This Cd-MOF can enantioselectively catalyze the addition of $\mathrm{ZnEt}_{2}$ to aromatic aldehydes (Scheme 1) with varying sizes $(0.8-2 \mathrm{~nm})$ and ee over $80 \%{ }^{70}$ While the performance of homogeneous catalyst in $\mathrm{ZnEt}_{2}$ addition does not depend on the substrate size, in the case of Cd-MOF increasing the size of the aldehyde leads to a decrease in the conversion. This observation is consistent with the operation of heterogeneous catalysis in the case of Cd-MOF.

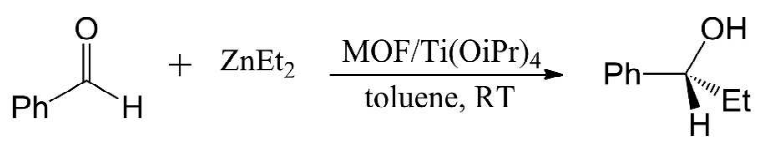

Scheme 1. MOF-catalyzed $\mathrm{ZnEt}_{2}$ addition to aromatic aldehyde.

A similar synthesis protocol was used for preparation of homochiral MOF $\left[\mathrm{Cd}_{3}((\mathrm{R})-6,6\right.$ 'dichloro-2,2'-dihydroxy-1,1'-binaphthyl-bipy $\left.)_{4}\left(\mathrm{NO}_{3}\right)_{6}\right] \cdot 7 \mathrm{MeOH} \cdot 5 \mathrm{H}_{2} \mathrm{O}$, followed by subsequent modification with $\operatorname{Ti}\left(\mathrm{O}^{i} \mathrm{Pr}\right)_{4} \cdot{ }^{71}$ The resulting Ti-containing Cd-MOF was highly enantioselective in the asymmetric $\mathrm{ZnEt}_{2}$ addition to aldehydes ( $>99 \%$ conversion and up to $90 \%$ ee). Interestingly, another material synthesized with the same ligand but from different source of $\mathrm{Cd}$ and crystallized in another space group did not catalyze the addition of $\mathrm{ZnEt}_{2}$ under identical conditions, even though it possesses permanent porosity and chiral dihydroxy groups. Authors believe that the lack of catalytic activity of the last system is a result of the steric congestion around chiral dihydroxy groups of the ligands which prevents the substitution of two isopropoxide groups of $\operatorname{Ti}\left(\mathrm{O}^{i} \mathrm{Pr}\right)_{4}$ by the binolate functionality.

Continuing with application of MOF as solid chiral catalysts, Lin and coworkers studied the performance of intermolecular $\left[\mathrm{Ti}(\mathrm{BINOLate})_{2}\right]$ complexes with chiral MOF $\left(\left[\mathrm{Zn}_{2}\left((\mathrm{R})-2,2^{\prime}-\right.\right.\right.$ dihydroxy-1,1'-binaphthyl-4,4',6,6'-tetrabenzoate)-(DMF) $\left.\left.\left(\mathrm{H}_{2} \mathrm{O}\right)\right] \cdot 2 \mathrm{EtOH} \cdot 4.3 \mathrm{DMF} \cdot 4 \mathrm{H}_{2} \mathrm{O}\right) \quad$ for $\mathrm{ZnEt}_{2}$ addition to aromatic aldehydes to form secondary alcohols. ${ }^{72}$ 1-Naphthaldehyde, 
benzaldehyde and its $4-\mathrm{Cl} / \mathrm{Br}$-substituted derivatives were used as substrates. In contrast to earlier results, only very modest enantioselectivity was observed in these reactions $(17.4 \%$ ee for benzaldehyde at $13 \mathrm{~mol} \%$ catalyst loading). The influence of the structure and cavity dimensions in the asymmetric induction ability is a general observation in heterogeneous enantioselective catalysts and is believed to arise from perturbations on the ideal geometry of the transition state in which the chiral active center is interacting with the substrates. If the transition state for optimal enantioselectivity transfer cannot fit inside the cavity, then asymmetric induction by the solid porous catalyst does not proceed. Modeling and computational calculations should be a powerful tool to understand and rationalize ee values in porous catalysts.

In support of this general assumption, a set of catalysts based on isoreticular chiral MOFs with the same non-interpenetrating framework but different open channel sizes was constructed from copper paddle-wheels and BINOL-derived tetracarboxylic acid bridging ligands by Lin et al. ${ }^{73}$ Postsynthetic grafting of the Ti(IV) complex onto these materials resulted in highly active catalysts for the addition of $\mathrm{ZnEt}_{2}$ to aromatic aldehydes. It was found that the ee of reaction is dependent on the channel sizes because of the competition between the enantioselective (BINOLate) $\operatorname{Ti}\left(\mathrm{O}^{i} \mathrm{Pr}\right)_{2}$-catalyzed reaction and the non-enantioselective background reaction and because of the different diffusion rates of the organic substrates through the open channels of varied sizes. $^{73}$

Similarly to the case of diethylzinc, Lin et al. demonstrated the catalytic activity of a combination of $\mathrm{Cu}$-containing chiral mesoporous MOFs and $\mathrm{Ti}\left(\mathrm{O}^{i} \mathrm{Pr}\right)_{4}$ for the addition of alkynylzinc to aromatic aldehyde leading to chiral unsaturated alcohol (Scheme 2). ${ }^{73}$ The $\mathrm{Cu}$ MOF under investigation affords almost total conversion for a wide range of aldehydes to chiral alcohols with ee up to $76 \%$. The crystal structure of the MOF was stable under the reaction conditions used for the catalytic alkynylzinc addition. The lower ee values observed 
for the MOF catalyst in comparison with homogeneous analogues were explained by the occurrence of a competing non-enantioselective reaction. ${ }^{73}$ Additional experiments with MOF indicated a significant background reaction catalyzed by non-chiral active sites to give the racemic alcohols. Apparently, these non-enantioselective sites are preferentially located in the most accessible parts of the crystallite (external surface or defects) since 1-naphthaldehyde as substrate affords products with much lower ee than those achieved with the other aromatic aldehydes. Thus, ee values depend on the sizes of the substrates and the MOF channels. Transport of large reagents and product molecules is of critical importance and this is a hallmark of reactions taking place in the internal voids of microporous solids.
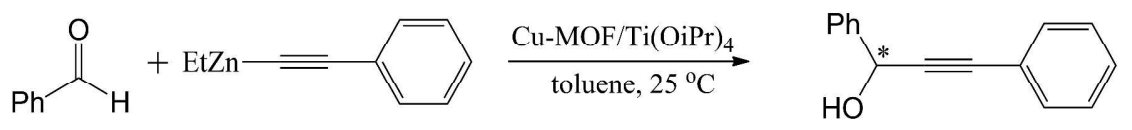

Scheme 2. Asymmetric alkynylzinc addition to aromatic aldehyde.

Wang and coworkers investigated the asymmetric addition of Grignard reagent to dienophile over porous homochiral MOF constructed from a serine derivate ligand [(S)-3-hydroxy-2(pyridin-4-ylmethylamino)propanoic acid] as linker bridged and chelated with copper atoms. ${ }^{27}$ Examined MOF catalyzed the 1,2-addition of cyclohexylmagnesium chloride to a range of oxocompound (Scheme 3) to give high adduct conversion with the ee value up to $99 \%{ }^{27}$ Interestingly, copper compounds usually promote 1,4 -addition of the Grignard reagent to $\alpha, \beta$ unsaturated ketones. ${ }^{128}$ This contrasting behavior suggests that the active sites in the Cu MOF catalysis are the organic linkers rather than the metal nodes. This possibility was supported by testing the outcome of reference reactions with either the free ligand or $\mathrm{CuCl}_{2}$. The free ligand could render the 1,2-addition product with $84 \%$ conversion and $51 \%$ of ee, while $\mathrm{CuCl}_{2}$ practically did not catalyze this organometallic addition. It was established that most of the operating active sites are present on the MOF surface and that the internal centers do not contribute due to the small pore size that limits the accessibility of substrates. ${ }^{27}$ Thus, it was 
concluded that reaction takes place on the catalyst surface rather than in the micropores. It was also shown that low reaction temperature $\left(-80^{\circ} \mathrm{C}\right)$ can improve the ee value but decreases the conversion.

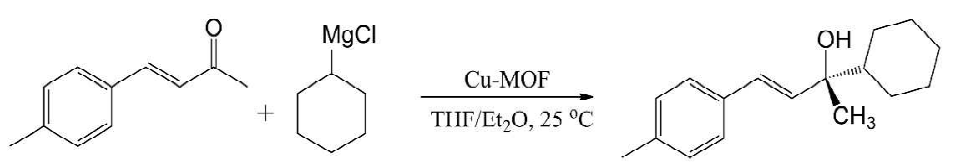

Scheme 3. 1,2-Addition of Grignard reagent to oxocompound.

\section{Ring opening of epoxides by alcohols}

Compounds containing vicinal ether and alcohol groups can be easily synthesized by alcohol addition to epoxides.

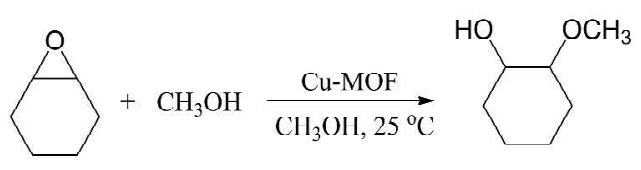

Scheme 4. Ring-opening reaction of epoxide with alcohol.

$\mathrm{Cu}$-containing MOFs have been reported as catalysts for alcoholysis of epoxides. Kim et al. studied the synthesis of MOF containing a tetranuclear $\mathrm{Cu}(\mathrm{II})$-btp units weakly connected by nitrate ions, as efficient, mild, and easily recyclable catalyst for the alcoholysis of epoxides. ${ }^{74}$ Under investigated conditions the regiochemistry of the ring opening depended on the electronic nature of the substrate rather than steric factors. The studied MOF showed conversion of cyclohexene oxide higher than $99 \%$ after 6 days and exhibited better performance than $\mathrm{Cu}\left(\mathrm{NO}_{3}\right)_{2}(55 \%)$ as homogeneous catalyst. The same authors have tested $\mathrm{Cu}$-containing MOF with btp-linkers and perchlorate/sulfate anions as catalyst for the alcoholysis of epoxides. ${ }^{75}$ Interestingly, while the activity of MOF with perchlorate anions in epoxide ring opening was comparable to homogeneous $\mathrm{Cu}\left(\mathrm{ClO}_{4}\right)_{2}, \mathrm{SO}_{4}{ }^{2-}$-containing MOF was practically inactive in this reaction in contrast to $\mathrm{CuSO}_{4}$. These results may be correlated with the need of coordinatively unsaturated sites acting as active centers. 
Lough et al. investigated activity of MOF containing $\mathrm{Cu}\left(\mathrm{H}_{2} \mathrm{O}\right)_{2}{ }^{2+}$ and $\mathrm{Cu}\left(\mathrm{NO}_{3}\right)_{2}$ units alternatively bridged by btp ligands with H-bonds between copper-bonded water and nitrate oxygen atoms in alcohol addition to epoxides. ${ }^{76}$ This catalyst was active at room temperature promoting the epoxide ring aperture of cyclic (Scheme 4, cyclohexene oxide was completely converted after 4 day) and acyclic epoxides (cis- and trans-2-butene oxides afford the corresponding products in 10 and 30 days, respectively). Another example of $\mathrm{Cu}-\mathrm{MOF}$ as a highly active and selective heterogeneous catalyst in the alcoholysis of epoxides was demonstrated by Baiker and coworkers. ${ }^{77}$ Activity of $\mathrm{Cu}($ bipy $)\left(\mathrm{H}_{2} \mathrm{O}\right)_{2}\left(\mathrm{BF}_{4}\right)_{2}$ (bipy) was comparable with homogeneous catalyst $\mathrm{Cu}\left(\mathrm{BF}_{4}\right)_{2} \cdot \mathrm{H}_{2} \mathrm{O}$ in the case of methanolysis, but the relative activity dropped significantly with increasing steric demands of the alcohol (in the sequence methanol $>i$-propanol $>$ tert-butanol), a trend that is compatible with the location of active sites inside the micropores of the MOF. Conversion and selectivity in transformation of styrene oxide with methanol to 2-methoxy-2-phenylethanol were over $90 \%$ at room temperature in only $2 \mathrm{~h}$. This result is significantly different to previous data on the activity of other $\mathrm{Cu}$-containing MOFs tested in the alcoholysis of epoxides. In contrast to the activity of $\mathrm{Cu}$ (bipy) $\left(\mathrm{H}_{2} \mathrm{O}\right)_{2}\left(\mathrm{BF}_{4}\right)_{2}$ (bipy), other $\mathrm{Cu}-\mathrm{MOF}$ were either inactive or required several days to complete the reaction under the same reaction conditions. ${ }^{77}$ The reaction could be extended to aliphatic and cycloaliphatic epoxides with good yields under mild conditions. Although structural water involved in H-bonding between two-dimensional sheets of the MOF framework may be partially replaced by methanol under reaction conditions, leaching test and recycling experiments demonstrated that MOF under investigation is a truly heterogeneous catalyst.

Dhakshinamoorthy et al. used porous Fe(btc) [iron(III)1,3,5-benzenetricarboxylate; $\left.\mathrm{C}_{9} \mathrm{H}_{3} \mathrm{FeO}_{6}\right]$ as a heterogeneous catalyst for the epoxide ring opening of various substrates under mild reaction conditions. ${ }^{129}$ Compared with heterogeneous, non-porous iron citrate and other 
related MOFs, $\left(\mathrm{Cu}_{3}(\mathrm{btc})_{2}\right.$ and $\left.\mathrm{Al}_{2}(\mathrm{bdc})_{3}\right), \mathrm{Fe}(\mathrm{btc})$ exhibited a superior performance derived from a combination of Lewis acid iron ions with one exchangeable coordination position and large porosity in terms of surface area and pore size. ${ }^{129}$ It was shown that steric encumbrance on the alcohol or impeded diffusion play an unfavourable role on the conversion. The rate of the ring-opening reaction of styrene oxide decreased as the size of the alcohol was increased, suggesting the location of active sites in micropores. ${ }^{129} \mathrm{Fe}(\mathrm{btc})$ was a truly heterogeneous catalyst and could be reused without a loss of activity.

Tanaka and Otani reported a chiral $\mathrm{Cu}-\mathrm{MOF}$ catalyzing kinetic resolution of styrene oxide by alcoholysis. ${ }^{78}$ This reaction was very sensitive to the structure of alcohol. Increasing substrate size results in a dramatic drop of conversion and enantioselectivity under the same reaction conditions. This trend was justified by a slower diffusion of bulky alcohols inside the pores of the MOF catalyst, where the active sites are predominantly located. The proposed mechanism of the kinetic resolution of styrene oxide was based on the predominant coordination of the $\mathrm{R}$ substrate enantiomer to the Lewis acid $\mathrm{Cu}$ site of chiral MOF (due to the steric congestion) followed by an attack by methanol to the $\alpha$-carbon atom of the substrate from the backside position to give (S)-product with inversion of stereochemistry. ${ }^{78}$

Rosseinsky et al. post-synthetically introduced acid sites into a chiral microporous amino acid-based MOFs [Ni(L-aspartate)bipy ${ }_{0.5}$ and $\mathrm{Cu}(\mathrm{L}$-aspartate $)$ bpe $\left._{0.5}\right]$ and tested the activity of the resulting heterogeneous catalysts in the asymmetric methanolysis of cis-2,3-epoxybutane. ${ }^{79}$ Ni-containing MOF was nonporous and exhibited lower activity than Cu-containing framework, which was able to uptake molecules of both methanol and propylene oxide. ${ }^{79}$ Almost complete lack of reactivity of (2,3-epoxypropyl)-benzene was considered an evidence in support of the assumption that the reaction occurs mainly inside the MOF pores. ${ }^{79}$

\section{Ring opening of epoxides by amines}


Vicinal amino alcohols ( $\beta$-alkanolamines) exhibit strong biological activity and can be found among beta blockers (propranolol, pindolol), amino acids (serine), hormones and neurotransmitters (epinephrine, norepinephrine). ${ }^{130,}{ }^{131}$ Epoxide ring opening by amines is a general reaction for the synthesis of these compounds and MOFs have been reported to be active solid catalysts for this process.

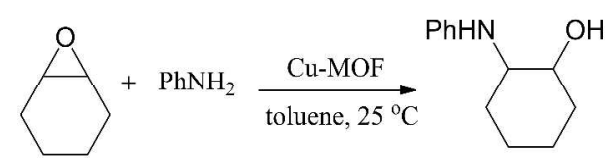

Scheme 5. Ring-opening reaction of epoxides with amines.

Tanaka et al. examined the catalytic activity of chiral MOF $\left[\mathrm{Cu}_{2}(\mathrm{bda})_{2}\left(\mathrm{H}_{2} \mathrm{O}\right)_{2}\right] \cdot \mathrm{MeOH} \cdot 2 \mathrm{H}_{2} \mathrm{O}$ for the asymmetric ring opening reactions of epoxides with aromatic amines. ${ }^{80}$ Optically active $\beta$ amino alcohol with a $54 \%$ yield and $45 \%$ ee value was obtained after 2 days at room temperature in toluene. Both the reactivity and enantioselectivity could be improved under solventless conditions (from 15 to $30 \%$ for yield, and from 43 to $50 \%$ for ee in cyclopentene oxide reaction with aniline).$^{80}$

Baiker and coworkers studied aminolysis of cyclohexene oxide with aniline using $\mathrm{Cu}$ (bipy) $\left(\mathrm{H}_{2} \mathrm{O}\right)_{2}\left(\mathrm{BF}_{4}\right)_{2}$ (bipy) as catalyst. ${ }^{77}$ The isolated yield of corresponding product over $\mathrm{Cu}$ MOF was $32 \%$ in $4 \mathrm{~h}$, which is lower than that achieved with the homogeneous catalyst $\mathrm{Cu}\left(\mathrm{BF}_{4}\right)_{2} \cdot \mathrm{H}_{2} \mathrm{O}(50 \%){ }^{77}$ The lower catalytic activity of the heterogeneous Cu-MOF catalyst was explained as due to diffusion limitations of reagents and products in the micropores of MOF (Scheme 5).

\section{Henry (nitroaldol) reaction}

$\beta$-Nitro alcohols are useful synthetic intermediates since they can be easily converted to nitroalkenes (by dehydration), $\alpha$-nitro ketones (oxidation), or $\beta$-amino alcohols (reduction), which are very useful for producing of fine chemicals. ${ }^{132} \beta$-Nitro alcohols can be obtained by 
nucleophilic attack of $\alpha$-carbanions derived from nitroalkanes onto reactive carbonylic groups (Nitroaldol or Henry addition).

Shi and $\mathrm{Wu}$ reported the synthesis of porous MOF constructed from two kinds of pyridine carboxylates and $\mathrm{Cu}(\mathrm{II})$ nodes. ${ }^{81}$ The copper sites located on the channel walls are accessible and having activity for the catalytic transformation of benzaldehyde and nitromethane to the corresponding nitro alcohol (Scheme 6). ${ }^{81}$ The yields achieved are remarkable compared with homogeneous catalysts $(85,47,0 \%$ yield of the product of 4-nitrobenzaldehyde reaction with nitromethane over MOF, $\mathrm{Cu}(\mathrm{OAc})_{2} \cdot \mathrm{H}_{2} \mathrm{O}$ and $\mathrm{Cu}\left(\mathrm{NO}_{3}\right)_{2} \cdot 3 \mathrm{H}_{2} \mathrm{O}$, respectively). ${ }^{81}$ Using different solvents (EtOH, $i$-PrOH, 1,4-dioxane and toluene), it was demonstrated that the active $\mathrm{Cu}^{2+}$ sites can be easily blocked by solvent molecules, and the substrates should compete with them during the reaction mechanism. Therefore, the best results were obtained under solvent-free conditions.

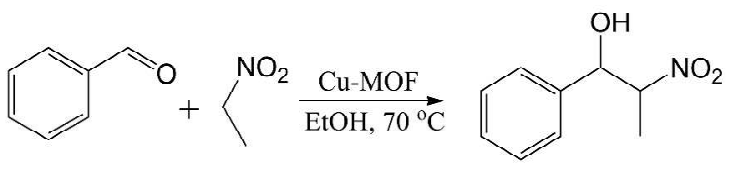

Scheme 6. Nitroaldol (Henry) reaction between benzaldehyde and nitromethane.

Huh and coworkers examined substrate size-dependence in the Henry reaction over basic DABCO-functionalized Zn-containing 3D-MOF (DABCO = 1,4-diazabicyclo[2,2,2]octane), which showed promising robustness and stability since it can be recycled several times without losing activity. ${ }^{82}$ The increase in nitroalkane size resulted in a decrease in conversion. Nitromethane $(5.20 \times 5.10 \AA)$, nitroethane $(5.50 \times 6.50 \AA), 1$-nitropropane $(5.52 \times 7.38 \AA)$, 2nitropropane $(6.75 \times 6.68 \AA)$, and nitrocyclohexane $(6.75 \times 8.92 \AA)$ were used as substrates. Conversion as well as TON (after $120 \mathrm{~h}$ ) decreased in the order of nitromethane $(80 \%, 48.2)>$ nitroethane $(34 \%, 20.5)>2$-nitropropane $(30 \%, 18.1)>1$-nitropropane $(19 \%, 11.4)>$ nitrocyclohexane $(12 \%, 7.2)$. Since nitromethane is less acidic $\left(\mathrm{pK}_{\mathrm{a}}=10.24\right)$ than nitroethane 
$\left(\mathrm{pK}_{\mathrm{a}}=8.60\right)$, 1-nitropropane $\left(\mathrm{pK}_{\mathrm{a}}=8.98\right)$, and 2-nitropropane $\left(\mathrm{pK}_{\mathrm{a}}=7.7\right)$, authors established that the higher conversion for nitromethane can be attributed to the size effect rather than the electronic effects. ${ }^{82}$ As commented earlier, this size dependency supports the internal location of the active sites, the activity being sensitive to diffusion of substrates through the pores.

\section{Ether formation by acetalization or alcohol addition to enone}

Kapteijn et al. developed a new strategy for the direct encapsulation of POM into chromiumcontaining MIL-101 using addition of PTA to the synthesis mixture of the corresponding MOF. ${ }^{83}$ This procedure resulted in the formation of the lacunary structures of the polyoxometalate, being active in dimethyl ether production from methanol. ${ }^{83}$ This catalytic activity contrasts with the low activity of the catalysts prepared via impregnation of POM in MIL-101, where the strong interaction between polyoxometalate and support deteriorates the catalytic performance. It was observed, however, that the selectivity to dimethyl ether was relatively low, a fact that was explained by the formation of longer chain hydrocarbons, which can be adsorbed into the MIL-101 cavities. ${ }^{83}$ Both selectivity to dimethyl ether and conversion of methanol over MOF increased with temperature.

Hupp and coworkers synthesized MOF-based N-heterocyclic carbene-like catalyst by using n-butyllithium to deprotonate tripodal imidazolate framework. ${ }^{84}$ This MOF was composed of a neutral linker containing three imidazole substituents coordinated to $\mathrm{Co}^{2+}$-ions to form two catenated, two-dimensional sheets. The catenation of the sheets creates accessible onedimensional channels. The resulting carbene-like catalyst attached to the MOF is able to promote the reaction of $\alpha, \beta$-unsaturated ketone with benzyl alcohol to the corresponding benzyl ether (Scheme 7). This MOF exhibits a faster reaction rate and higher yield than the traditional homogeneous $\mathrm{N}$-heterocyclic carbene catalyst 1,3-bis-(2,4,6-trimethylphenyl)imidazole. ${ }^{84}$ Varying the size of the alcohol in the conjugate addition did not change the reaction rate, even 
when the size of the alcohol exceeded the diameter of the MOF pores. As a result, the catalytic reactions must occur exclusively on the external surface of the MOF crystals and, therefore, improved performance presumably could be achieved if a new MOF featuring larger channels could be synthesized to enable interior sites to catalyze conjugate addition or by decreasing size of MOF particles. This reasonable possibility is still to be proved experimentally.

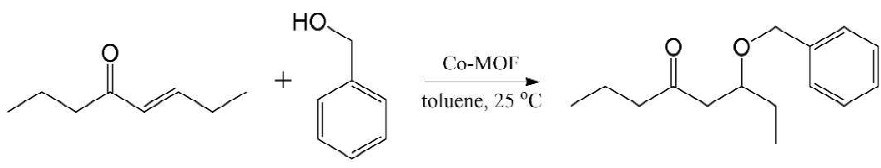

Scheme 7. Conjugate addition of benzyl alcohol to $\alpha, \beta$-unsaturated ketone.

Acetals and ketals are used as protecting groups for carbonyl groups since they are stable with respect to oxidizing and reducing agents and to hydrolysis by bases. ${ }^{133}$ Some of these compounds are used as fragrances or intermediates in multistep organic synthesis.

Monge et al. tested the catalytic activity of the hybrid two-dimensional germanate framework $\left[\mathrm{C}_{6} \mathrm{H}_{10}\left(\mathrm{NH}_{2}\right)_{2} \mathrm{Ge}_{3} \mathrm{O}_{6}\right]^{134}$ and $\mathrm{Yb}$ succinate polymeric frameworks in the acetalization of benzaldehyde with trimethyl orthoformate (Scheme 8). ${ }^{85}$ The Yb-MOF catalysts were stable both in water and in organic solvents and exhibited a reversible temperature-induced phase transition between two polymorphs without losing or gaining any solvent/guest molecule. ${ }^{85}$ While the reaction of benzaldehyde with trimethyl orthoformate in the presence of $\alpha$-polymorph of Yb-MOF (stable below $130{ }^{\circ} \mathrm{C}$ ) produced the corresponding dimethyl acetal in good yield $(90 \%$ after $5 \mathrm{~h})$ at moderate temperature $\left(60{ }^{\circ} \mathrm{C}\right)$, Ge-MOF exhibited even higher activity (100\% yield of target product after $2 \mathrm{~h}$ at $\left.40{ }^{\circ} \mathrm{C}\right)$. Few Incontaining MOFs $\left(\left[\operatorname{In}_{2}(\mathrm{OH})_{3}(\mathrm{bdc})_{1.5}\right], \quad\left[\operatorname{In}_{2}(\mathrm{OH})_{2}(\mathrm{bdc})_{2}(\mathrm{phen})_{2}\right], \quad\left[\operatorname{In}(\mathrm{btc})\left(\mathrm{H}_{2} \mathrm{O}\right)(\mathrm{bipy})\right]\right.$, and $\left[\operatorname{In}(\mathrm{BTC})\left(\mathrm{H}_{2} \mathrm{O}\right)(\right.$ phen $\left.)\right]$, (phen= $O$-phenantroline) were also tested in the same reaction. ${ }^{135}$ Using $10 \mathrm{~mol} \%$ of MOFs as catalysts, yields up to $70 \%$ (TOF up to $380 \mathrm{~h}^{-1}$ ) were achieved after $4 \mathrm{~h}$ under relatively mild reaction conditions $\left(60-70^{\circ} \mathrm{C}\right)$. 
Garcia et al. carried out room temperature acetalization of series of aromatic and aliphatic aldehydes with methanol using $\mathrm{Cu}_{3}(\mathrm{btc})_{2}, \mathrm{Fe}(\mathrm{btc})$ and $\mathrm{Al}_{2}(\mathrm{bdc})_{3}$ in good yields without the need of water removal. ${ }^{136} \mathrm{Cu}_{3}(\mathrm{btc})_{2}$ showed the best catalytic activity and its performance as acetalization catalyst was comparable or even better than those of other conventional homogeneous and heterogeneous catalysts such as $\mathrm{Cu}\left(\mathrm{NO}_{3}\right)_{2}, \mathrm{ZnCl}_{2}$, zeolites (NaY, USY, BEA) and clay (Bentonite). ${ }^{136}$ As it was demonstrated by the poisoning effect of bases and by comparison with the activity of copper nitrate in the homogeneous phase, the catalytically active sites for this reaction are the metal ions acting as Lewis acids. The structural stability of MOF, reusability and heterogeneity of this catalytic system was also demonstrated. ${ }^{136}$

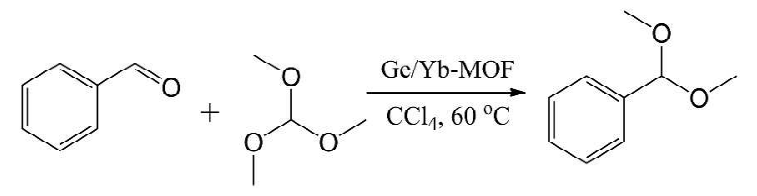

Scheme 8. MOF-catalyzed acetalization of benzaldehyde

$\mathrm{Su}$ et al. reported size-selective catalysis in ketalization reactions over few isostructural 3D phosphine-based MOFs. ${ }^{86}$ The MOFs possess 1D chiral channels with $\mathrm{Cu}_{2}$ (pyridyl diphosphine $)_{2}$ dimeric subunits and $\mathrm{Br}^{-}, \mathrm{Cl}^{-}$or $\mathrm{PF}_{6}^{-}$counteranions. $\mathrm{PF}_{6}^{-}$-containing framework showed significantly higher yield (93\%) than the $\mathrm{Br}^{-}$analogue $(67 \%)$ for the ketalization of 2butanone (Scheme 9). ${ }^{86}$ This was explained by the easier accessibility of the substrate to the $\mathrm{Cu}(\mathrm{I})$ coordination sphere, because $\mathrm{PF}_{6}{ }^{-}$is a weakly coordinating anion. ${ }^{86}$ Different substrates with varied sizes and shapes (2-butanone, cyclohexanone, acetophenone and benzophenone) were used to test the size selectivity. Upon increasing substrate size, a significant decrease in the yields was observed (for $\mathrm{Br}^{-}$-containing MOF from 67 to 36 and $17 \%$ for 2-butanone, cyclohexanone and acetophenone, respectively ${ }^{86}$. No conversion was detected when using bulky benzophenone as substrate. Both leaching tests and XRD patterns before and after the catalytic run suggest the stability of the framework structure during the reaction. ${ }^{86}$ In addition, 
the obtained MOFs were stable in usual solvents such as chloroform, dichloromethane, acetonitrile, toluene, water, alcohols, and no obvious loss of catalytic activity for three successive catalytic runs was observed. ${ }^{86}$

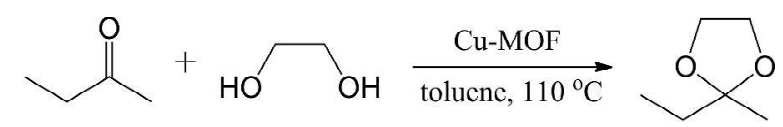

Scheme 9. Ketalization of 2-butanone by ethylene glycol.

Hatton et al. ${ }^{137}$ studied the catalytic activity of MIL-101(Cr) and MIL-101(Cr)/PTA composites in dimethyl acetal formation from benzaldehyde and methanol at room temperature. Almost total benzaldehyde conversion (93-95\%) was observed within $24 \mathrm{~h}$ in reactions catalyzed by the MIL101/PTA composites $\left(\mathrm{TOF}=18-22 \mathrm{~min}^{-1}\right.$ ), while MIL-101 after the same reaction time exhibited $80 \%$ conversion with quite poor efficiency $\left(\mathrm{TOF}=0.19 \mathrm{~min}^{-1}\right)$. It was found, that activation energies for dimethyl acetal formation from benzaldehyde catalyzed by MIL101/PTA composites $(63-67 \mathrm{~kJ} / \mathrm{mol})$ were close to that determined for acetalization of benzaldehyde catalyzed by $\mathrm{HClO}_{4}(60 \mathrm{~kJ} / \mathrm{mol})$ and dimethyl acetal formation from methanol and acetaldehyde catalyzed by Amberlyst-15 (72 kJ/mol).

Synthesis of carbonyl compounds by Diels Alder reaction, Meinwald rearrangement and Pechmann condensation

Aldehydes and ketones are widely used as solvents, perfumes, flavouring agents, for producing plastics, dyes, pharmaceuticals. ${ }^{138,139}$ For instance, some steroids (progesterone, testosterone, cortisone, and aldosterone), flavorings (carvone, vanillin), or vitamins (retinal) are aldehydes or ketones.

The Diels-Alder cycloaddition dienes with dienophiles is a simple synthetic route to polycyclic compounds containing carbonyl group as side chain. In 1998-2000 Aoyama and coworkers examined polyphenoxide MOFs such as $\mathrm{Zr}_{2}\left(\mathrm{O}^{t} \mathrm{Bu}\right)_{4}(\mathrm{abr}), \mathrm{Ti}_{2}\left(\mathrm{O}^{i} \mathrm{Pr}\right)_{2} \mathrm{Cl}_{2}(\mathrm{abr})$, 
$\mathrm{Al}_{2} \mathrm{Cl}_{2}(\mathrm{abr})$, and $\mathrm{Al}_{2}\left(\mathrm{O}^{i} \mathrm{Pr}\right)_{2}(\mathrm{abr})$ as heterogeneous Lewis-acid catalysts for the Diels-Alder reaction of 1,3-cyclohexadiene with acrolein (Scheme 10). ${ }^{87-89}$ Although the crystal structures of studied materials were unknown, these MOFs were highly porous and had unsaturated metal centers available for substrate coordination. In Diels-Alder reaction, these MOFs exhibited activities higher than their corresponding homogeneous analogues $\left(\mathrm{Zr}\left(\mathrm{O}^{t} \mathrm{Bu}\right)_{2}, \operatorname{Ti}\left(\mathrm{O}^{i} \mathrm{Pr}\right)_{2} \mathrm{Cl}_{2}\right)$ with high stereoselectivities (endo/exo ratios higher than 99/1 for Ti/Al-containing MOFs and over 95/5 for Zr-containing catalyst). Recently, Hatano and Ishihara designed MOFs with conformationally flexible chiral pockets suitable for different types of substrates (so-called 'tailor-made' chiral supramolecular catalysts), leading to high values of anomalous substrateselectivity and/or stereoselectivity in Diels-Alder reaction. ${ }^{90}$ The corresponding materials were prepared in-situ from chiral 3,3'-disubstituted binaphthols and biphenols, arylboronic acid and $\mathrm{B}\left(\mathrm{C}_{6} \mathrm{~F}_{5}\right)_{3} .{ }^{90}$ They can promote both the anomalous endo- and anomalous exo-selective DielsAlder reaction of cyclopentadiene with $\alpha, \beta$-unsaturated oxocompounds.

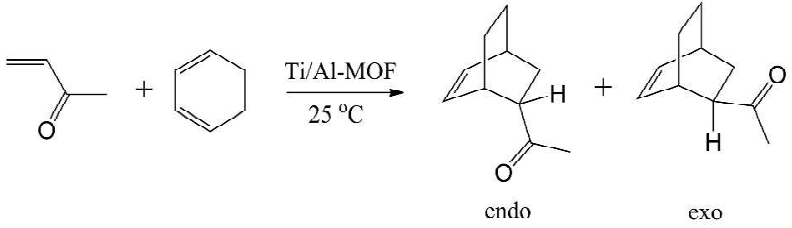

Scheme 10. Diels-Alder reaction between 1,3-cyclohexadiene and acrolein.

Kim et al. used $\mathrm{NH}_{2}-\mathrm{MIL}-101(\mathrm{Al})$ as catalyst for Meinwald rearrangement of epoxide to aldehyde (Scheme 11). ${ }^{91}$ The reaction proceeded with reasonable yield (75-80\%) only when using epoxides containing aromatic group in $\alpha$-position..$^{91}$ This reactivity pattern was explained as arising from the additional stabilization of intermediate state due to the resonance effect caused by the aromatic ring. ${ }^{91}$ The specific reactivity of aromatic epoxides is, however, surprising since mesoporous aluminosilicates are able to catalyze the rearrangement of a wide range of epoxides including aliphatic ones without substrate selectivity. Garcia and coworkers reported the activity of $\mathrm{Fe}(\mathrm{btc})$ in rearrangement of styrene oxide (with $23 \%$ conversion after 6 
h at $100{ }^{\circ} \mathrm{C}$ and $84 \%$ selectivity to phenylacetaldehyde) and norbornene oxide (with $6 \%$ conversion after $6 \mathrm{~h}$ at $70{ }^{\circ} \mathrm{C}$ and $85 \%$ selectivity to norbornone). ${ }^{92}$

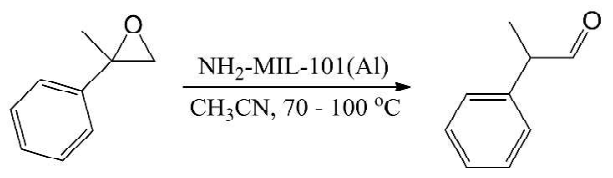

Scheme 11. Meinwald rearrangement of epoxide.

Čejka et al. studied the catalytic activity of $\mathrm{Cu}_{3}(\mathrm{btc})_{2}$ and $\mathrm{Fe}(\mathrm{btc})$ in the Pechmann condensation of different phenols (resorcinol, pyrogallol, and naphthol) with ethyl acetoacetate to produce coumarines (Scheme 12) and compared their performance with that of acidic zeolites. ${ }^{93}$ Resorcinol and pyrogallol as substrates provided poor yields of corresponding coumarines over MOFs in comparison with zeolites. ${ }^{93}$ Strong adsorption of these phenolic substrates (containing two or more OH-groups) on the active sites of MOFs was proposed as responsible for this poor performance that is accompanied by the partial destruction of the framework of the catalyst. ${ }^{93}$ In contrast, zeolites exhibited low conversions for the transformation of relatively bulky naphthol to the target product, while $\mathrm{Cu}_{3}(\mathrm{btc})_{2}$ and $\mathrm{Fe}(\mathrm{btc})$ were highly active (94-98\% conversion with $100 \%$ selectivity) and the crystallinity of the framework was preserved in the process. ${ }^{93}$

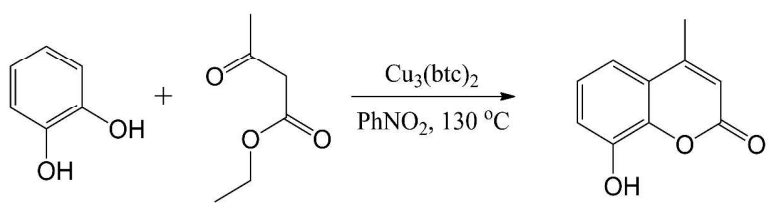

Scheme 12. Pechmann condensation of resorcinol with ethyl acetoacetate.

Friedel-Crafts acylation is a general organic reaction widely used for the synthesis of specialty compounds in fine chemistry. ${ }^{140}$ Recently, a few examples of Friedel-Crafts acylation by benzoyl chloride resulting in formation of aromatic ketones have been presented. Acylation of $p$-xylene (Scheme 13) was selected as model reaction and the catalytic activity of $\mathrm{Cu}_{3}(\mathrm{btc})_{2}$, 
MIL-100(Fe), MIL-100(Cr) catalysts compared to that of zeolites Beta and USY as conventional solid acids. ${ }^{94}$ While zeolites exhibited similar conversions as $\mathrm{Cu}_{3}(\mathrm{btc})_{2}$ and MIL$100(\mathrm{Cr})$, the total conversion of p-xylene was achieved over MIL-100(Fe) in the temperature range of $100-130^{\circ} \mathrm{C} .{ }^{94}$ It was also found that activation temperatures from 10 to $200{ }^{\circ} \mathrm{C}$ do not practically affect the activity of MIL-100(Fe) in this reaction. ${ }^{94}$ Phan et al. used IRMOF-8 ${ }^{95}$ and ZIF-8 ${ }^{96}$ as catalysts for the benzoylation of toluene and anisole, respectively. Over $50 \%$ conversion of toluene and $80 \%$ selectivity to 4-methylbenzophenone were achieved over IRMOF- 8 at $80{ }^{\circ} \mathrm{C}$. Increasing the reaction temperature in the sequence $80-90-100{ }^{\circ} \mathrm{C}$ led to a significant enhancement in reaction rate $(50,72,75 \%$ conversion, respectively), while selectivity to $p$-isomer remained almost unchanged. As for ZIF-8 the presence of electrondonating group (methoxy) in the benzoyl chloride accelerated the reaction with anisole $(87 \%$ conversion after $6 \mathrm{~h}$ at $\left.120{ }^{\circ} \mathrm{C}\right)$ in comparison with unmodified benzoyl chloride $(82 \%$ conversion), while reaction involving 4-chlorobenzoyl chloride (containing electronwithdrawing group) gave $73 \%$ conversion of substrate. ${ }^{96}$ However, the selectivity to $p$-isomer in the reaction using 4-methoxybenzoyl was lower (60-70\%) compared with benzoyl chloride (90-95\%) and 4-chlorobenzoyl chloride (75-90\%). Formation of a single regioisomer is the target of this catalytic process and there are certain cases, in which acid microporous solids can control the product distribution favoring the para isomer through the operation of shape selectivity.<smiles>O=C(Cl)c1ccc(-c2ccccc2)cc1</smiles>

Scheme 13. Friedel-Crafts acylation

Synthesis of hydroxy carbonyl compounds by dehydration of glucose, Mukaiyama-aldol and Aldol reaction 
The hydroxy aldehydes and ketones are used as synthetic intermediates and building blocks for the synthesis of oxiranes, allylic alcohols, diols and other intermediates in syntheses of natural products and compounds of pharmaceutical interest (such as antidepressants, HIVprotease inhibitors and antitumorals). ${ }^{141}$ The $\alpha$-hydroxy carbonyl compounds are structural subunits of sugars and $\beta$-hydroxy- $\alpha$-amino acids.

Hensen et al. reported the activity of PTA/MIL-101 composite for the selective dehydration of fructose and glucose to 5-hydroxymethylfurfural (Scheme 14) in ionic liquids. ${ }^{97} \mathrm{Cr}^{3+}$-centers in MIL-101 were practically inactive for the dehydration of carbohydrates. ${ }^{97}$ Free PTA also gave very poor yield of the target product $(8 \%) .{ }^{97}$ In addition, the activity of MOF catalyst was higher than that of the $\mathrm{CrCl}_{2}$. Increasing PTA loading from 12 to 27 and $30 \%$, increased the 5hydroxymethylfurfural yield from 20 to 58 and $61 \%$, respectively. ${ }^{97}$ When protons in the catalyst were neutralized by titration with sodium hydroxide, almost no yield was detected, indicating that protons derived from PTA/MIL-101 are the active sites for carbohydrates dehydration. ${ }^{97}$ In fact, the limited reusability of PTA/MIL-101 in ionic liquids was attributed to the exchange of PTA protons by the cations of the ionic liquid. For this reason, DMSO was proposed as alternative solvent and the yield of 5-hydroxymethylfurfural in this medium was $63 \%$, while blank experiment provided only $18 \%$ yield. $^{97}$

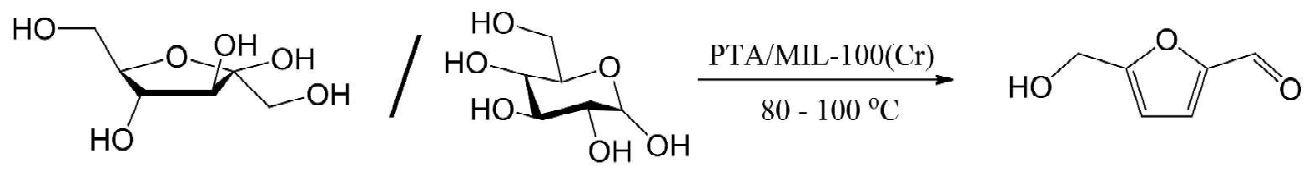

Scheme 14. Dehydration of fructose/glucose to 5-hydroxymethylfurfural.

Long and coworkers tested sodalite-type MOF $\mathrm{Mn}_{3}\left[\left(\mathrm{Mn}_{4} \mathrm{Cl}\right)_{3}(\mathrm{btt})_{8}\left(\mathrm{CH}_{3} \mathrm{OH}\right)_{10}\right]_{2}$ as catalysts for the Mukaiyama-aldol reaction between aldehyde and silyl enolate (Scheme 15). ${ }^{98}$ It was found that this Mn-containing MOF exhibited shape-selectivity since benzaldehyde and relatively small silyl enolate yielded $63 \%$ of the target product at room temperature. The use of 
4-tert-butylbenzaldehyde reduced the conversion under the same conditions to only $24 \%$. In the same way the use of larger cyclic silyl enolates for the reaction with benzaldehyde leads to practically no conversion. The $\mathrm{Mn}^{2+}$ centers were active even in the presence of a highly coordinating solvent such as DMF since addition of the examined MOF accelerated benzaldehyde conversion at $-45{ }^{\circ} \mathrm{C}$ from $8 \%$ to $51 \%{ }^{98}$

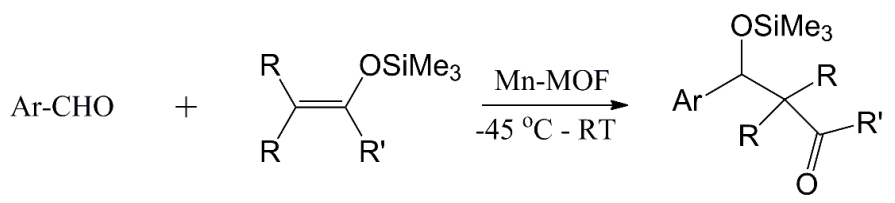

Scheme 15. Mukaiyama-aldol reaction.

Duan et al. synthesized a Cd-containing MOF with 1,3,5-tris(4-carboxyphenyl)benzene as ligands in the presence of L-bcip (bcip: N-tert-butoxy-carbonyl-2-(imidazole)-1-pyrrolidine) as chiral inductor. ${ }^{99}$ This Cd-MOF was active in aldol reaction between aromatic aldehyde and cyclohexanone (Scheme 16), achieving up to $97 \%$ yield of the corresponding aldol with reasonable ee value about $60 \%{ }^{99}$

Kim and coworkers incorporated L-proline units into MIL-101 to design chiral organic ligands ((S)-N-(pyridin-3-yl)-pyrrolidine-2-carboxamide and (S)-N-(pyridin-4-yl)-pyrrolidine2-carboxamide), in which 3- or 4-pyridyl units were coordinated to the open metal coordination sites of the framework. ${ }^{100}$ The resulting chiral MOFs exhibited catalytic activity in the asymmetric aldol reactions between aromatic aldehydes and ketones (Scheme 16) with 60-90\% yields of corresponding aldol products and enantioselectivity for R-isomers up to $80 \%$. ${ }^{100}$ For the aldol reaction between $p$-nitrobenzaldehyde and acetone, the modified MIL-101 showed higher activity (66\% yield, $69 \%$ ee after $24 \mathrm{~h})$ in comparison with the parent MOF (10\% yield, $0 \%$ ee after $120 \mathrm{~h}$ ), and with the selectivity of the chiral ligand (29\% ee) under homogeneous conditions. ${ }^{100}$ Size-selectivity was studied to prove that the reactions do not proceed exclusively on the external surface of the MOF. For this purpose, the authors selected 
the condensation between acetone and a bulky aldehyde whose molecular size is larger than the window of the MOF (5-formyl-1,3-phenylene-bis-3,5-di-tert-butylbenzoate). ${ }^{100}$ While in the presence of chiral ligand the reaction was completed within $36 \mathrm{~h}$, only $5 \%$ of the target product was observed in the presence of MOF after the same period of time.

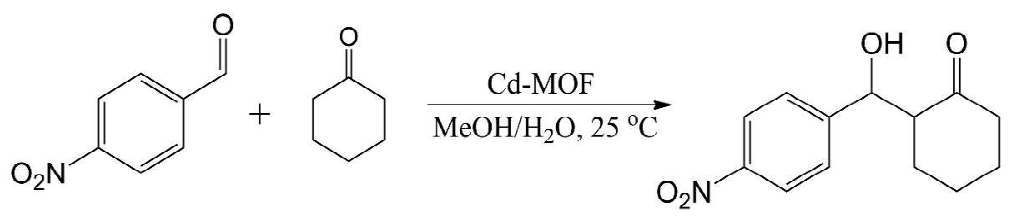

Scheme 16. Aldol reaction between 4-nitrobenzaldehyde and cyclohexanone.

Telfer et al. presented a strategy based on the use of thermolabile protecting groups for the incorporation of organocatalytic moieties into MOFs during their synthesis. ${ }^{101}$ A proline moiety was derivatized with Boc (Boc: tert-butoxycarbonyl) protecting group that was later removed after MOF synthesis, resulting in the formation of a $\mathrm{Zn}$-containing IRMOF exhibiting high catalytic activity for asymmetric aldol reactions of 4-nitrobenzaldehyde with acetone/cyclopentanone. ${ }^{101}$ The bulky Boc group also prevented framework interpenetration, thereby rendering an open MOF with larger void size that allows activation relatively large substrates. ${ }^{101}$ The ee of the aldol product was $29 \%$ for the condensation with acetone, and 3-14 $\%$ for reactions with cyclopentanone. The low ee values in asymmetric aldol reactions over these MOFs were explained by a combination of the following factors: i) the lack of organization of the reaction transition state due to the various possible locations of proline with respect to the framework, ii) the absence of accessible hydrogen bond donors on the catalytic unit, and iii) large intraporous space that allows the access of the nucleophile from both sides of the intermediate. ${ }^{101}$ It was also demonstrated that IRMOF containing proline moiety is responsible for the observed catalytic activity since the reaction did not proceed after filtration of the catalyst or in the presence of MOF containing protecting groups.

\section{Synthesis of carboxylic acid derivatives}


A large variety of carboxylic acid derivatives (such as esters, hydroxy esters, amides, nitriles) is widely used as fine chemicals for a wide range of applications including flavors, anesthetics, lubricants, as intermediates in different asymmetric synthesis, etc. ${ }^{142},{ }^{143}$ In this section we will focus on substituted esters and unsaturated nitriles, while amides will be considered in a specific section devoted to amines.

\section{Esterification}

Kapteijn et al. used direct encapsulation of POMs into MIL-101(Cr) ${ }^{83}$ The resulting PTAMIL-101(Cr) samples were active for the esterification of $n$-butanol with acetic acid. The best catalytic activity was achieved by the sample containing $20 \mathrm{wt} \%$ polyoxometalate encapsulated sample (TON more than 3000 and a TOF value around $11 \mathrm{~h}^{-1}$ ). Although the initial activity of MOF was lower than that of free POM in homogeneous phase, the encapsulated sample showed TONs similar to those of other supported catalysts. ${ }^{83}$

Kim and coworkers investigated chiral Zn-containing MOFs for the transesterification of ester (Scheme 17). ${ }^{104}$ Zinc ions in the framework are held together by carboxylate groups and a bridging oxo oxygen to form a $\mathrm{Zn}_{3}\left(\mu_{3}-\mathrm{O}\right)$ (carboxylate) $)_{6}$ building unit. It can be further linked by coordinating $\mathrm{Zn}$ ions with pyridyl groups to generate 2D layers with large (13.4 $\AA$ ) chiral one-dimensional channels. The reactivity of such MOFs was examined for the size-selective transesterification of 2,4-dinitrophenyl acetate with ethanol, 2-butanol, neopentanol and 3,3,3triphenyl-1-propanol. ${ }^{104}$ While the product yield for the reaction of ethanol was $77 \%$ at room temperature, transesterification with bulkier alcohols occurred under identical reaction conditions at much slower or even negligible reaction rates. ${ }^{104}$ These observations are compatible with the assumption that the reaction proceeds mainly in the channels of MOF. Also, the reaction of 2,4-dinitrophenyl acetate with a racemic 1-phenyl-2-propanol in the 
presence of $\mathrm{D}$ - or the enantiomorphic L-MOF produced the corresponding esters with $8 \%$ ee in favor of S- or R- enantiomer, respectively.

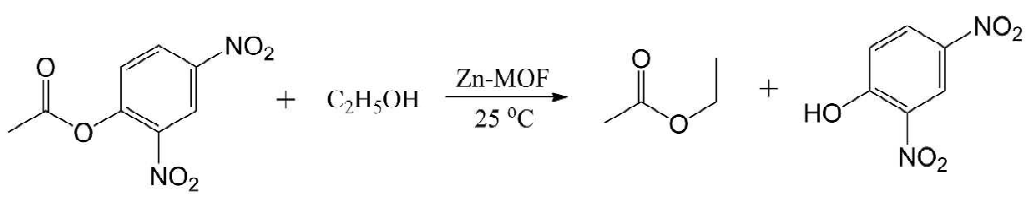

Scheme 17. Transesterification of ester by alcohol.

Bats et al. used imidazolate framework ZIF-8 for the transesterification of rapeseed oil to produce alkyl esters. ${ }^{105}$ The reaction of vegetable oil with methanol at $200{ }^{\circ} \mathrm{C}$ catalyzed by ZIF8 led to the complete conversion into monoglycerides. The activity of MOF depended on the nature of the alcohol involved in the reaction (methanol, ethanol, 1-propanol, 1-butanol, 2propanol, tert-butanol). ${ }^{105}$ While it decreased with the chain length for linear alcohols, branched alcohols exhibited almost no reactivity. ${ }^{105}$

Very recently, Cu-containing MOFs $\left[\left\{\mathrm{Cu}_{2}(\mathrm{Glu})_{2}(\mu-\mathrm{bpa})\right\} \cdot\left(\mathrm{CH}_{3} \mathrm{CN}\right)\right]_{\mathrm{n}}$ and $\left[\left\{\mathrm{Cu}_{2}(\mathrm{Glu})_{2}(\mu-\right.\right.$ bpp) $\left.\} \cdot\left(\mathrm{C}_{3} \mathrm{H}_{6} \mathrm{O}\right)\right]_{\mathrm{n}}$ (bpp: 1,3-bis-(4-pyridyl)-propane) were studied by Huh and coworkers for the transesterification of various esters with methanol. ${ }^{106}$ While bpp-containing MOF was less active and promoted a slow conversion of phenyl acetate, bpa-containing MOF was found to effectively catalyze the complete reaction of phenyl acetate with methanol under neutral conditions. It was shown that bpa-containing MOF can be reused up to 20 times without any significant deterioration of its catalytic activity. The reaction rates over this catalyst were very sensitive to the properties of the electronic groups on the phenyl moiety of the substrate. The substrates with the electron-withdrawing substituents (4-nitrophenyl acetate/benzoate) undergo faster transesterification, while those with electron-donating groups (4-methylphenyl acetate/benzoate) exhibit slower reaction rates. ${ }^{106}$

\section{Mukaiyama-aldol reaction}


Ding et al. reported assembling of polymeric chiral titanium complexes using linked bisBINOL ligands, which were active in carbonyl-ene reaction to afford corresponding $\alpha$-hydroxy ester (Scheme 18). ${ }^{107}$ The linkers between two BINOL units have significant impact on the enantioselectivity of the reaction between $\alpha$-methylstyrene and ethyl glyoxylate. The reaction proceeded smoothly at room temperature over Ti-MOF prepared using as linker a paraphenylene ligand to give (S) $\alpha$-hydroxy ester in $91 \%$ yield with $94.4 \%$ ee after $48 \mathrm{~h}^{107}$ Lowering the reaction temperature to $0{ }^{\circ} \mathrm{C}$ resulted only in a decrease in the yield $(85 \%$ after $120 \mathrm{~h}$ ) without an improvement of the enantioselectivity. ${ }^{107}$ The reaction carried out under solvent-free conditions afforded the (S)-product with $75 \%$ yield with essentially identical enantioselectivity. ${ }^{107}$ In contrast, the Ti-MOF prepared using the meta-phenylene linker showed poor catalytic activity and enantioselectivity (32\% yield and $9.8 \%$ ee after 48 h) under the same experimental conditions. ${ }^{107}$ The best-performing Ti-MOF catalyst was that obtained from a BINOL dimer, which gave the (S)-product in $99 \%$ yield and $96.5 \%$ ee after $30 \mathrm{~h}$ at room temperature. ${ }^{107}$ Unfortunately, this Ti-MOF based on BINOL gradually dissolved in the reaction mixture under solvent-free conditions and cannot be considered due to its lack of structural stability.

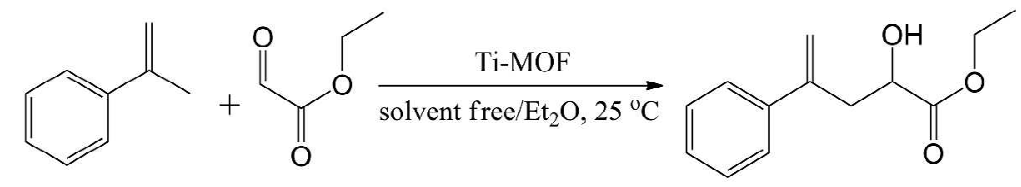

Scheme 18. Carbonyl-ene reaction

The same authors reported the superior catalytic performance of chiral titanium complexes with bridged multitopic BINOL ligands when compared to that in homogeneous phase. ${ }^{108}$ Like in the previous work, the authors underlined the importance of the spacers between two neighbor BINOL units of the ligands. The introduction of an electron-withdrawing substituent to the backbone of BINOL could significantly improve the catalytic activity of the material in 
carbonyl-ene reaction due to the increase in the Lewis acid strength of titanium complexes. ${ }^{108}$ The titanium complexes bonded to MOF afforded $\alpha$-hydroxy esters in high yields (99\%) with excellent enantioselectivities (95-98 \% ee) under various reaction conditions. ${ }^{108}$ Diethyl ether was a suitable solvent for running the heterogeneous reaction because of lower solubility of MOFs in this solvent. ${ }^{108}$ However, it would be interesting to survey further this system to check if it is truly heterogeneous since there are some hints such as the gradual drop in both activity and enantioselectivity with each catalyst recycling and the pale yellow color of the reaction mixture. It could indicate some leaching of the active sites.

Tanabe and Cohen reported the functionalization of parent UMCM-1-NH 2 having large pores and high surface area with two different metal binding groups that were finally metalated with $\mathrm{Fe}^{3+}$ and $\mathrm{Cu}^{2+}$ ions. ${ }^{102}$ The resulting $\mathrm{Fe}^{3+}$ and $\mathrm{Cu}^{2+}$ complexes attached to the UMCM-1$\mathrm{NH}_{2}$ structure were catalytically active MOFs for the Mukaiyama-aldol reaction (Scheme 19). The Fe-containing catalyst showed approximately $58 \%$ conversion at room temperature for benzaldehyde and 1-naphthaldehyde over three catalytic cycles, while blank experiments gave less than $10 \%$ conversion under the same conditions. ${ }^{102}$

Kaskel and coworkers reported the synthesis of chiral MOFs with wide-open pores (>30 $\AA$ ) using chiral trifunctional linkers consisting on btb and multinuclear zinc clusters. ${ }^{103}$ The materials obtained were tested in the Mukaiyama aldol reaction of aldehyde with 1-methoxy-1(trimethylsiloxy)-2-methyl-1-propene (Scheme 19) and their catalytic activity was compared with that of a homogeneous analogue (zinc nitrate tetrahydrate), MOF-177 having large accessible pore volume, and MIL-101. ${ }^{103}$ The highest initial conversion in the reaction with benzaldehyde was found for MIL-101, but it was surpassed by that of one of the btb-containing MOFs after one day reaction. The performance of zinc nitrate tetrahydrate was unexpectedly low presumably due to the low solubility in n-heptane. The poorest conversion in the Mukaiyama aldol reaction was obtained over MOF-177. ${ }^{103}$ Using larger 1-naphthaldehyde as 
substrate for the Mukaiyama aldol reaction with silyl enol ether resulted in a drop of the conversion rates for all catalysts. ${ }^{103}$ The MOF constructed with 1,3,5-tri $\{4-[2-(4-i s o p r o p y l-2-$ oxooxazolidin-3-yl)]benzoate $\}$ benzene ligands showed the best performance (97\% conversion), but in contrast to the conversion of benzaldehyde, the gap in activity of this wide open MOF and other catalysts was significant (65\% - zinc nitrate tetrahydrate, $61 \%$ - MOF177 and $69 \%$ - MIL-101), showing the benefits of large pore sizes to favor diffusion at room temperature. ${ }^{103}$ A leaching test proved the heterogeneity of the reaction, XRD patterns before and after catalysis proved that the framework of all MOF catalysts remains intact. ${ }^{103}$

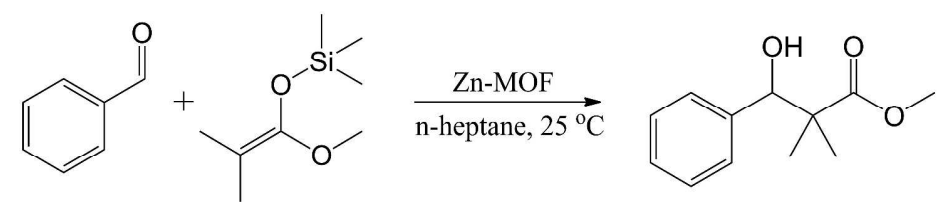

Scheme 19. Mukaiyama aldol reaction to produce hydroxy ester

\section{Knoevenagel condensation}

Knoevenagel condensation is the nucleophilic addition of an active hydrogen compound (containing $-\mathrm{C}(\mathrm{O}) \mathrm{R},-\mathrm{CN},-\mathrm{COOR}$ groups in $\alpha$-position to $-\mathrm{CH}_{2}-$ ) to a carbonyl group followed by dehydration. This reaction is a convenient route to obtain $\alpha, \beta$-conjugated esters and nitriles. Due to the amenable reaction conditions and analysis, this condensation is also one of the preferred model reactions to evaluate the activity of solid catalysts.

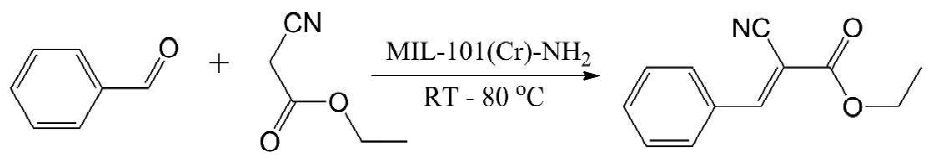

Scheme 20. Knoevenagel condensation.

Several strategies of postfunctionalization of MIL-101 with amine groups to produce solid base catalysts have been reported and tested for the Knoevenagel condensation. Ferey and coworkers grafted ethylenediamine onto the exchangeable coordination positions of $\mathrm{Cr}^{3+}$ in 
MIL-101(Cr) and used the resulting MOF for the condensation of benzaldehyde with ethyl cyanoacetate (Scheme 20). ${ }^{110}$ The dehydrated MIL-101(Cr) exhibited $32 \%$ conversion at 80 ${ }^{\circ} \mathrm{C} .{ }^{110}$ The catalytic performance of ethylenediamine grafted MIL-101 (98\% conversion and 99 $\%$ selectivity to ethyl trans- $\alpha$-cyanocinnamate, TOF $=328 \mathrm{~h}^{-1}$ ) was noticeably better than that of SBA-15 modified with 3-aminopropyltrialkoxysilane (75\% conversion and $94 \%$ selectivity, TOF $=32 \mathrm{~h}^{-1}$ ), even though the content of free amine groups in MOF was significantly lower than that of mentioned mesoporous sieve. ${ }^{110}$ The condensation of benzophenone with malononitrile to form bulky 1,1-dicyano-2,2-diphenylethene took place much more sluggishly, suggesting that the modified MIL-101(Cr) exhibits size-dependence catalytic activity characteristic of processes taking place inside the micropores. ${ }^{110}$ However, the different chemical reactivity of benzophenone with respect to benzaldehyde must also account for the decrease in the product yield and comparison must be taken cautiously. Similarly, Ahn et al. used post-synthesis functionalization of MIL-101(Cr) using diethylenetriamine. ${ }^{111}$ It was established that the catalyst amount significantly affects the yield of benzylidene malononitrile in benzaldehyde with malononitrile condensation. ${ }^{111}$ Increasing the amount of catalyst from 10 to 20 and $50 \mathrm{mg}$ per $2 \mathrm{mmol}$ of substrate led to an increase in the yield of the target product from 31 to 60 and $90 \%$ at room temperature, respectively.

Hartmann and Fischer examined amino-functionalized MOFs based on the large-pored Fe/Al-MIL-101 for the condensation of benzaldehyde with malononitrile and ethyl cyanoacetate yielding benzylidene malononitrile and ethyl $\alpha$-cyanocinnamate, respectively. ${ }^{112}$ These MOFs were stable under the reaction conditions and more active (yields of the corresponding products were $\geq 90 \%$ at $80{ }^{\circ} \mathrm{C}$ ) than solid bases such as $\mathrm{MgO}$ and hydrotalcite even without the assistance of polar or protic solvents. ${ }^{112}$ No appreciable difference in activity was observed between Fe-MIL-101- $\mathrm{NH}_{2}$ and Al-MIL-101- $\mathrm{NH}_{2}$, indicating that the framework metal should play only a minor role in the Knoevenagel condensation. ${ }^{112}$ 
Farrusseng et al. reported a quantitative structure-activity relationship of functionalized ZIFtype materials and showed an elegant strategy to control their hydrophility-hydrophobicicty by post-synthetic modification. ${ }^{113}$ Modification of the environment of catalytic sites through the creation of hydrophobic environment surrounding the active centers resulted in an increase of the reaction rate for Knoevenagel condensation (from about 200 to $1500 \mathrm{~h}^{-1}$ of TOF). ${ }^{113}$ It was shown that the contact angle, a measure of the hydrophilicity, is directly correlated to the catalytic activity of MOFs. ${ }^{113}$ Authors also concluded that under investigated conditions the catalytic activity is not controlled by internal diffusion inside the pores.

Recently, Corma and coworkers introduced a defective catalytic functionality to the MOFs demonstrating that such defects or occluded metal oxides can be responsible for the activity in Knoevenagel condensation reaction. ${ }^{114}$ Thus, different preparation routes of MOFs may result in different concentration of defects and different reactivity. On the other hand, the presence of defect introducing a second catalytic functionality (Lewis acid sites) can activate undesired side reactions. Therefore, it was concluded, that the presence of defects must be taken into account when acid-promoted reactions are undesired.

Crystalline Co-containing zeolite imidazolate framework ZIF-9 having narrow pores was used as an efficient heterogeneous catalyst for the room temperature liquid phase Knoevenagel reaction between benzaldehyde and malononitrile to afford benzylidene malononitrile. ${ }^{115}$ The reaction could only occur in the presence of the catalyst without any contribution from leached active species in the reaction solution. The ZIF-9 catalyst is reusable several times without a significant loss of catalytic activity ( $100 \%$ conversion after $3 \mathrm{~h}$ in $1^{\text {st }}-3^{\text {rd }}$ runs). However, it is obvious considering the relative dimensions of pores and product molecules that the reaction occurred on the external surface of the ZIF-9 particles, which makes the prospects of using this catalyst questionable. 
Zhou et al. constructed multifunctional (containing both Lewis acid and base sites) MOF PCN-124 from $\mathrm{Cu}$ paddlewheel motifs and pdai (containing pyridine and amide groups) as ligand. ${ }^{116}$ The resulting material showed catalytic activity in a tandem one-pot deacetalizationKnoevenagel condensation reaction. ${ }^{116}$ The first step of the reaction was the acid-catalyzed deacetalization of dimethoxymethylbenzene to give benzaldehyde. The second step gave benzylidene malononitrile through the Knoevenagel reaction between benzaldehyde and malononitrile. PCN-124 was found to be a highly active cooperative catalyst for this tandem one-pot reaction with a TON of higher than $190 .{ }^{116}$ It can be easily recovered from the reaction medium and reused at least for three cycles without significant loss of activity $(100,96.6,91.9$, $96.7 \%$ yield of benzylidene malononitrile in $1^{\text {st }}-4^{\text {th }}$ run, respectively). As commented earlier, due to the flexibility in design and post-functionalization, MOFs offer a large potential for embedding various active sites, implementing multifunctional catalysis and making possible the development of tandem reactions. Another example of tandem transformation employed $\mathrm{NH}_{2}$-MIL-101(Al) as a bifunctional, site-isolated Lewis acid-Brønsted base heterogeneous catalyst. ${ }^{91} \mathrm{NH}_{2}$-MIL-101(Al) exhibits activity for consecutive Meinwald rearrangement of epoxides and Knoevenagel condensation of the formed aldehyde and malononitrile with remarkable substrate selectivity (up to $95 \%$ ). ${ }^{91}$ In control experiments, $\mathrm{AlCl}_{3}$ failed to promote the Knoevenagel condensation and an analogue of the organic linker present in $\mathrm{NH}_{2}$-MIL101(Al) did not affect the epoxide transformation. ${ }^{91}$

Kapteijn et al. reported the use of MIL-101(Cr) with incorporated POMs as solid catalysts for the Knoevenagel condensation of benzaldehyde and ethyl cyanoacetate. ${ }^{83}$ This catalyst was active both in apolar (toluene) and in polar (DMF, ethanol) solvents, showing excellent performance (TOF up to about $700 \mathrm{~h}^{-1}$ ). ${ }^{83}$

The same authors used a MOF with non-coordinated amino groups (IRMOF-3) as stable solid basic catalyst in the Knoevenagel condensation of ethyl cyanoacetate and ethyl 
acetoacetate with benzaldehyde. ${ }^{117}$ IRMOF-3 exhibited high conversion (about $65 \%$ at $40{ }^{\circ} \mathrm{C}$ ) with a $100 \%$ selectivity to the condensation product. ${ }^{117}$ The IRMOF-3 catalyst was stable during reaction and reusable without a significant loss in activity. ${ }^{117}$

Kitagawa and coworkers performed the Knoevenagel condensation catalyzed by $\mathrm{Cd}(4-$ btapa $\left.)_{2}\left(\mathrm{NO}_{3}\right)_{2}\right] \cdot 6 \mathrm{H}_{2} \mathrm{O} \cdot 2 \mathrm{DMF}$ to demonstrate its selective catalytic properties as a solid base. ${ }^{118}$ The condensation of benzaldehyde with active methylene compounds of different molecular size (malononitrile, ethyl cyanoacetate, and tert-butyl cyanoacetate) occurred with different conversions (98, 7 and $0 \%$ at room temperature, respectively). ${ }^{118}$ Based on this size dependence, the authors proposed that the reaction proceeds in the channels and not on the external surface of the catalyst. ${ }^{118}$ This seems to be questionable since other factors, particularly large differences in substrate acidity, must also play a role on the observed yield.

Opanasenko and coworkers reported the rare example of Lewis acid-catalyzed Knoevenagel condensation of cyclohexane carbaldehyde and benzaldehyde with malononitrile, methyl cyanoacetate, and ethyl acetoacetate. ${ }^{119}$ The catalytic behavior of $\mathrm{Cu}_{3}(\mathrm{btc})_{2}$ and $\mathrm{Fe}(\mathrm{btc})$ was compared with that of zeolites BEA and TS- $1 .{ }^{119}$ High yields of $\alpha, \beta$-unsaturated nitriles were achieved over the $\mathrm{Cu}_{3}(\mathrm{btc})_{2}$ in the reaction involving malononitrile, especially at a relatively low reaction temperature $\left(100 \%\right.$ yield at $80{ }^{\circ} \mathrm{C}$, no evidence for leaching of the active sites was obtained), in contrast to non-active zeolites ${ }^{119}$ The reactivity of active methylene compounds decreased in the order malononitrile $>$ methyl cyanoacetate $>$ ethyl acetoacetate, but the reactivity of cyclohexane carbaldehyde was higher or equal to the reactivity of benzaldehyde. ${ }^{119}$ The selectivity changed in the order malononitrile $>$ methyl cyanoacetate $\approx$ ethyl acetoacetate, benzaldehyde $>$ cyclohexane carbaldehyde. ${ }^{119}$ At higher reaction temperature $\left(130{ }^{\circ} \mathrm{C}\right)$, the activity of the catalysts also decreased in the order $\mathrm{Cu}_{3}(\mathrm{btc})_{2}>$ $\mathrm{Fe}(\mathrm{btc}) \geq \mathrm{BEA} \geq \mathrm{TS}-1 .{ }^{119}$ It has been proposed that, due to the perfect match in distances, a 
single molecule of malononitrile can be activated by two $\mathrm{Cu}$ atoms located in neighbor paddlewheels of the same cavity of $\mathrm{Cu}_{3}(\mathrm{btc})_{2}$.

\section{Synthesis of amines and amides via Aza-Michael addition, Friedländer condensation, Beckmann rearrangement and Biginelli reaction}

Amines and amides have numerous applications including production of propellants, pesticides, fungicides, herbicides, dyes, drugs. ${ }^{144}$ They are also used as corrosion inhibitors, antioxidants, protective agents, anticlinging and waterproofing agents for textiles, developers in photography, insect repellents. Derivatives of pyrimidone are the basis of many biological molecules. $^{145,146}$

Garcia and coworkers established that $\mathrm{Al}_{2}(\mathrm{bdc})_{3}, \mathrm{Cu}_{3}(\mathrm{btc})_{2}$ and $\mathrm{Fe}(\mathrm{btc})$ are active and selective catalysts for the polymethylation of aromatic amine (in particular, 2,4diaminotoluene) with dimethyl carbonate (Scheme 21). ${ }^{120} \mathrm{~N}$-methylation prevailed over carbamoylation even though they are competing parallel processes. ${ }^{120}$ Blank experiment provided $21 \%$ of amine conversion mainly to the para-monomethyl isomer. MOFs accelerated the rate of methylation at higher temperatures, but only $\mathrm{Al}_{2}(\mathrm{bdc})_{3}$ gave almost exclusively methylated products. ${ }^{120}$ The observed product distribution indicated that 2,4-diaminotoluene reacts with dimethyl carbonate in the presence of MOFs giving initially mono N-methylated products (mainly the para-isomer). It was shown that $\mathrm{N}$-methylation always precedes the carbamoylation (side reaction). It means, the selectivity to methylated products is always 100 $\%$, since carbamoylation should be considered as consecutive reaction, occurring only for $\mathrm{N}$ methyl derivatives probably due to their higher nucleophilicity. ${ }^{120} \mathrm{Al}_{2}(\mathrm{bdc})_{3}$ was chosen as catalyst for the methylation of other amines with various substituents. The reaction of aniline with dimethyl carbonate led to $89 \%$ conversion and $96 \%$ selectivity to methylated products. ${ }^{120}$ Since the strong electron withdrawing effect of the nitro group decreases the reactivity of the nitro aniline, relatively low conversion was obtained for this substrate. So far as ortho- 
substitution with respect to the amino group makes difficult dimethylation due to steric hindrance, 2,4-dichloroaniline and 2,4-dimethylaniline yielded the corresponding mono Nmethyl derivatives with high selectivity without any carbamoylation. $^{120}$ orthoPhenylenediamine was also subjected to polymethylation without carbamoylation. In the case of aliphatic cyclohexyl amine as well as n-hexylamine, methylation was accompanied with significant percentages of carbamoylated products. ${ }^{120}$ In addition, $\mathrm{Al}_{2}(\mathrm{bdc})_{3}$ was not totally stable in the presence of aliphatic amines and partial destruction of MOF structure takes place gradually during the course of reaction, due to the considerably higher basicity of aliphatic amines compared with aromatic amines. ${ }^{120}$ With aromatic amines as substrates, $\mathrm{Al}_{2}(\mathrm{bdc})_{3}$ can be reused with minor activity decrease.
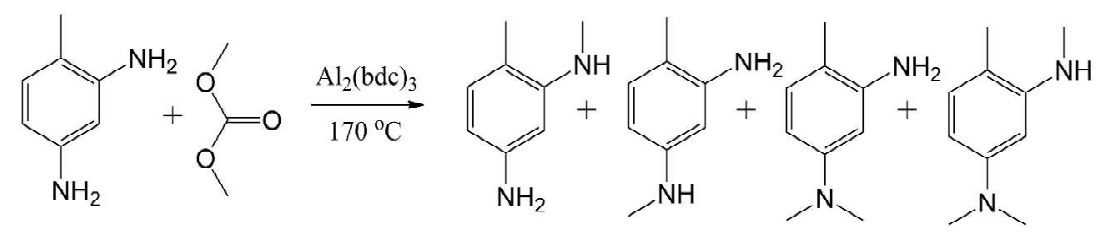

Scheme 21. Polymethylation of 2,4-diaminotoluene with dimethyl carbonate

Phan et al. reported the use of MOF-199 as a recyclable heterogeneous catalyst for the azaMichael reaction of benzylamine with ethyl acrylate to form ethyl 2-(benzylamino)acetate (Scheme 22). ${ }^{121}$ While the use of 5 mol \% of MOF-199 gave $89 \%$ conversion, decreasing catalyst concentration resulted only in a minor decrease in the reaction rate: conversions of 88 $\%$ and $84 \%$ could still be achieved at catalyst concentrations of 2.5 and $1 \mathrm{~mol} \%$, respectively, under the same reaction conditions. ${ }^{121}$ The selectivity to the mono-addition product remained almost unchanged, being over $98-99 \%$ in the catalyst concentration range of $1-5 \mathrm{~mol} \%{ }^{121}$ It was found that pyridine acts as a poison of the catalytic sites, leading its presence to a dramatic drop in the reaction rate, with only $46 \%$ conversion achieved. ${ }^{121}$ Pyridine poisoning is attributed to a competitive blocking of the MOF-199 Lewis acid sites by strongly adsorbed pyridine. $^{121}$ The MOF-199 catalyst could be reused several times without a significant 
degradation in catalytic activity. ${ }^{121}$ The aza-Michael reaction proceeds only in the presence of the MOF-199 catalyst, and there was no contribution from leached species present in the liquid phase. $^{121}$

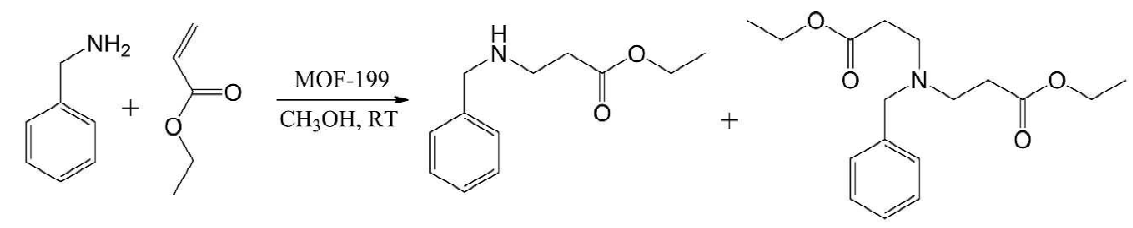

Scheme 22. Aza-Michael reaction.

Hatton and coworkers synthesized hybrid materials of the MIL-101(Cr) and PTA, with subsequent testing in the three-component condensation of benzaldehyde, 2-naphthol, and acetamide to produce substituted amide (Scheme 23) under microwave irradiation. ${ }^{147}$ Almost complete conversion of the reactants was observed after 5 min. However, precipitation of PTA was observed during the course of the reaction and catalyst recovery by filtration was problematic ( $<30 \mathrm{wt} \%$ of initial amount). ${ }^{147}$ No detectable product formation was observed without the catalysts and replacement of the MIL-101/PTA catalyst by $1-3 \%$ of the homogeneous PTA resulted in lower yields compared with the MIL101/PTA system.

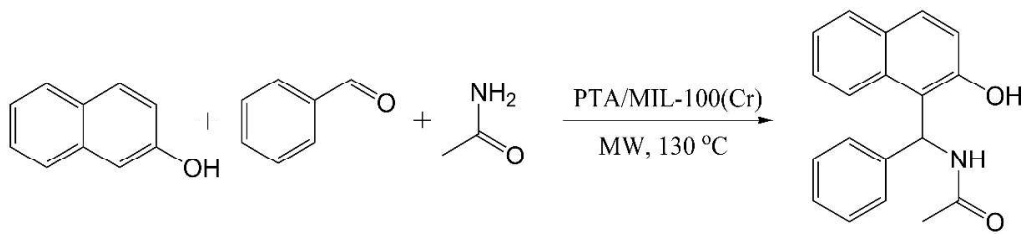

Scheme 23. Condensation of benzaldehyde, 2-naphthol and acetamide.

The catalytic activity of $\mathrm{Cu}_{3}(\mathrm{btc})_{2}$ and $\mathrm{Fe}(\mathrm{btc})$ for the Beckmann rearrangement of a series of aromatic and non-aromatic oximes (cyclohexanone, acetophenone, indanone, camphor oximes) was studied by Čejka and coworkers and compared with zeolites Beta and USY. ${ }^{122}$ The activity of the catalysts for the rearrangement of cyclohexanone oxime (Scheme 24) increased in the order $\mathrm{Fe}(\mathrm{btc})<\mathrm{Cu}_{3}(\mathrm{btc})_{2} \approx$ Beta $<\mathrm{USY} .{ }^{122}$ When using acetophenone oxime having almost the same kinetic diameter as cyclohexanone oxime, but containing an aryl substituent that exhibits 
a better migrating aptitude than the alkyl group, much higher conversions of the initial substance were achieved over all used catalyst, except $\mathrm{Fe}(\mathrm{btc}) .^{122}$ The conversions of cyclohexanone and acetophenone oximes over $\mathrm{Cu}_{3}(\mathrm{btc})_{2}$ were 11 and $80 \%$, respectively. ${ }^{122}$ The selectivity to the corresponding amides or lactams was close to $100 \%$ over both MOF catalysts. The advantages that MOFs can offer versus zeolites as heterogeneous catalysts for the synthesis of fine chemicals are nicely illustrated by the fact that while zeolite Beta was completely inactive in the Beckmann rearrangement of the most bulky camphor oxime into 1,8,8-trimethyl-2-azabicyclo[3.2.1] octan-3-one, $\mathrm{Cu}_{3}(\mathrm{btc})_{2}$ exhibited $80 \%$ conversion. $^{122}$ In general, while zeolites showed higher activity in the transformation of relatively small nonaromatic oxime (cyclohexanone oxime), $\mathrm{Cu}_{3}(\mathrm{btc})_{2}$ demonstrated comparable or even higher activity for the transformation of aromatic and bulky aliphatic oximes (acetophenone, indanone, camphor oximes). This probably is a reflection of the larger pore sizes of MOFs compared to zeolites, favoring easier diffusion of reactants and products within the internal voids.

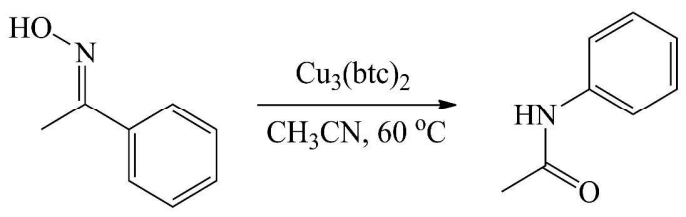

Scheme 24. Beckmann rearrangement of cyclohexanone oxime.

Čejka et al. have reported on the catalytic activity of $\left[\mathrm{Cu}_{3}(\mathrm{btc})_{2}\right]$ for the synthesis of quinolines by the Friedländer reaction of 2-aminobenzophenone (or 2-amino-5chlorobenzophenone) and acetylacetone under mild reaction conditions. ${ }^{148-150}\left[\mathrm{Cu}_{3}(\mathrm{btc})_{2}\right]$ exhibited highly improved catalytic performance (80\% yield of 1-(2-methyl-4-phenylquinolon-3-yl)-ethanone) in comparison with H-BEA (38 \%) and (Al)SBA-15 (36 \%).

Wang and coworkers tried to use porous homochiral $\mathrm{Cu}$-containing MOF constructed from a serine derivative as linker for the Biginelli reaction of benzaldehyde, urea and ethyl 
acetoacetate to give corresponding dihydropyrimidinone (Scheme 25$).{ }^{27}$ Despite the excellent yield reached $\left(90 \%\right.$ at $\left.40{ }^{\circ} \mathrm{C}\right)$, authors did not observe any enantioselectivity in the product distribution. The supernatant from used MOF was inactive to promote the reaction, confirming that the $\mathrm{Cu}$-containing MOF is acting as heterogeneous catalyst. ${ }^{27}$

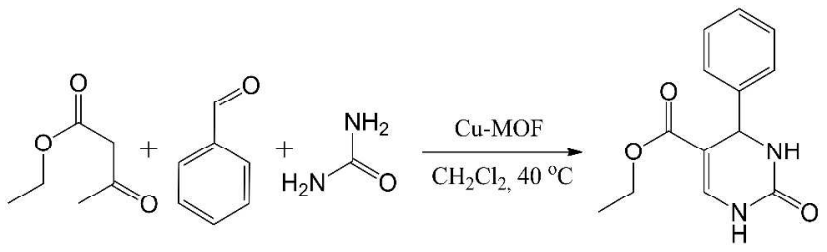

Scheme 25. Biginelli reaction.

Chorghade et al. used $\mathrm{Cu}_{3}(\mathrm{btc})_{2}$ for the synthesis of pyrimidine-chalcones by aldol condensation of substituted acetophenones and ethyl 1-formyl-1,2,3,6-tetrahydro-4-methyl-2(oxo/thioxo)-6-phenyl pyrimidin-5-carboxylates. ${ }^{151}$ Since condensation was initiated with the formation of an enolate ion, the Lewis acidity of $\mathrm{Cu}_{3}(\mathrm{btc})_{2}$ was enhanced by adding of concentrated $\mathrm{H}_{2} \mathrm{SO}_{4}$. Addition of $\mathrm{H}_{2} \mathrm{SO}_{4}$ facilitates formation of enolates by protonation. It was confirmed that sulfuric acid only enhanced the performance of $\mathrm{Cu}_{3}(\mathrm{btc})_{2}$ and did not catalyze the reaction in the absence of MOF.

Terpenoids formation by isomerization of a-pinene oxide, cyclization of racemic citronellal and Prins reaction

Terpenoids are the largest group of natural compounds derived from terpenes usually by moving/adding/losing of carbon fragments (methyl group) or oxygen atoms. Many terpenoids have biological activity and are used for the treatment of human diseases. As the largest class of natural products, terpenoids have a variety of roles in mediating antagonistic and beneficial interactions among organisms. Plant terpenoids are under investigation due to their antibacterial, antineoplastic, and other pharmaceutical functions. ${ }^{152}$

Garcia et al. tested a series of $\mathrm{Fe}^{3+}$-containing porous $\mathrm{MOF}$, including the commercial $\mathrm{Fe}(\mathrm{btc}), \mathrm{MIL}-88 \mathrm{~B}\left(\mathrm{Fe}_{3} \mathrm{O}(\mathrm{bdc})_{3} \mathrm{X}, \mathrm{X}=\mathrm{Cl}, \mathrm{OH},\right), \mathrm{MIL}-88 \mathrm{C}\left(\mathrm{Fe}_{3} \mathrm{O}(\mathrm{ndc})_{3} \mathrm{X}, \mathrm{X}=\mathrm{Cl}, \mathrm{OH},\right)[\mathrm{ndc}=$ 
1,6-naphthalenedicarboxylate], MIL-100 $\left(\mathrm{Fe}_{3} \mathrm{O}(\mathrm{btc})_{2} \mathrm{X}, \quad \mathrm{X}=\mathrm{Cl}, \mathrm{OH}\right)$, and MIL-127 $\left(\mathrm{Fe}_{6} \mathrm{O}_{2}(\mathrm{tazb})_{3} \mathrm{X}_{2}, \quad \mathrm{X}=\mathrm{Cl}, \mathrm{OH}\right)\left[\mathrm{tazb}=3,3^{\prime}, 5,5^{\prime}-\right.$ azobenzenetetracarboxylate $]$ for the rearrangement of $\alpha$-pinene oxide to camphonelal and isopinocamphone (Scheme 26). ${ }^{92}$ Catalytic performance of MOFs has been compared with that of $\mathrm{Fe}^{3+}$-exchanged Y-zeolite as well as some homogeneous $\left(\mathrm{ZnCl}_{2}, \mathrm{Cu}\left(\mathrm{NO}_{3}\right)_{2}, \mathrm{Al}\left(\mathrm{NO}_{3}\right)_{3}\right)$ Lewis acids. Besides isomerization to oxo-compounds, Lewis acid sites can also induce the ring opening of epoxide to verbenol, which further undergoes rearrangement to give isopinocamphone (Scheme 26). ${ }^{92}$ The reaction rate was higher for homogeneous catalysts, but MOFs showed a higher selectivity towards target products (camphonelal and isopinocamphone). When comparing the relative catalytic performance of various catalysts, MIL-100(Fe) has one of the highest TON value (14.3) being comparable with that of Fe-Y zeolite (18.3) and notably higher than those of the other solids $(0.9-7.0)$ and even homogeneous catalysts such as $\mathrm{ZnCl}_{2}(3.5)$ and $\mathrm{Cu}\left(\mathrm{NO}_{3}\right)_{2}(9.1) .{ }^{92} \mathrm{Fe}(\mathrm{btc})$ can be used for the isomerization of pinene oxide to campholenic aldehyde and isopinocamphone in the absence of solvent for few catalytic cycles but with decreasing activity due to a partial deactivation of the catalyst. In principle, described protocol could also be applied for the rearrangement of other epoxides.

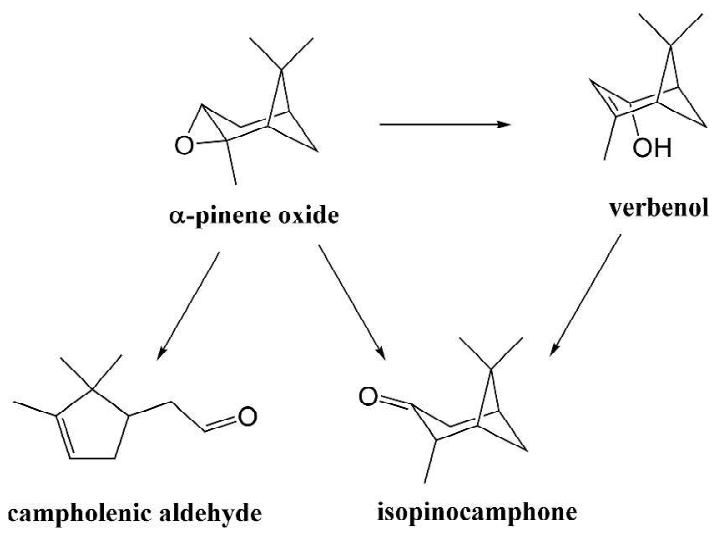

Scheme 26. Isomerization of $\alpha$-pinene oxide promoted by MOFs

De Vos et al. described another example of terpenoid synthesis. ${ }^{123} \alpha$-Pinene oxide transformation to campholenic aldehyde and cyclization of citronellal to isopulegol isomers 
(Scheme 27) were performed using as solid catalysts $\mathrm{Cu}_{3}(\mathrm{btc})_{2}$ synthesized in different conditions. ${ }^{123}$ It was shown that synthetic precursors of $\mathrm{Cu}_{3}(\mathrm{btc})_{2}\left(\mathrm{H}_{3}\right.$ btc and $\left.\mathrm{Cu}\left(\mathrm{NO}_{3}\right)_{2}\right)$ are not suitable catalysts. The organic ligand had generally a low activity in the isomerization reactions and there was no evidence that protonated benzenetricarboxylic acid, for example, at the surface of $\mathrm{Cu}_{3}(\mathrm{btc})_{2}$ or at defects in its structure, would significantly contribute to the overall acid activity. ${ }^{123}$ When $\mathrm{Cu}_{3}(\mathrm{btc})_{2}$ is compared with $\mathrm{Cu}\left(\mathrm{NO}_{3}\right)_{2}$, it was seen that the activity of the MOF Lewis acid sites is lower than that of $\mathrm{Cu}\left(\mathrm{NO}_{3}\right)_{2}$, but their selectivity towards the target aldehyde is higher for $\mathrm{Cu}_{3}(\mathrm{btc})_{2}$ (from 65 to $85 \%$ for $\mathrm{Cu}\left(\mathrm{NO}_{3}\right)_{2}$ and $\mathrm{Cu}_{3}(\mathrm{btc})_{2}$, respectively). ${ }^{123}$ It was established that different synthesis procedures did not influence much the catalytic results. ${ }^{123}$ Experiments using $\mathrm{Cu}_{3}(\mathrm{btc})_{2}$ with different crystal sizes, as well as the values of experimentally determined activation energies, indicated that reactant diffusion does not limit the reaction rates. In contrast, the nature of the solvent exerts a considerable influence on the selectivity and activity of $\mathrm{Cu}_{3}(\mathrm{btc})_{2}$ as catalyst for the $\alpha$-pinene oxide transformation to campholenic aldehyde. ${ }^{123}$ Regarding the selectivity of the reaction, 1,2-dichloroethane was the most suitable of all the solvents tested (1,2-dichloroethane, ethyl acetate, methanol, acetonitrile, toluene). ${ }^{123} \mathrm{~A}$ high selectivity was also reached in acetonitrile. The highest reaction rate was obtained in ethyl acetate, followed by 1,2-dichloroethane and methanol, while the lowest were determined for acetonitrile and toluene. ${ }^{123}$ The effect of solvents on the reaction rate and product selectivity was explained by competitive adsorption and relative acidity of the solvents. Strong adsorption of toluene on $\mathrm{Cu}_{3}(\mathrm{btc})_{2}$ was detected during preliminary sorption experiments, a fact that can explain the low rates observed in this solvent. ${ }^{123}$ Surprisingly, no methanolysis of $\alpha$-pinene oxide was detected in methanol. ${ }^{123}$ Possible side reactions during cyclization of citronellal (subsequent etherification, cracking, or dehydration of pulegols ${ }^{153}$ were also not observed in different solvents (toluene, 1,2-dichloroethane, chlorobenzene). ${ }^{123}$ The selectivity to desired isopulegols remained constant between 65 and $69 \%$ till complete 
conversion. Comparison with other $\mathrm{Cu}$ catalysts (zeolite $\mathrm{Cu}-\mathrm{Y}, \mathrm{Cu}\left(\mathrm{NO}_{3}\right)_{2}, \mathrm{Cu}(\mathrm{OAc})_{2}, \mathrm{CuCl}_{2}$, $\left.\mathrm{Cu}(\mathrm{OTf})_{2}, \mathrm{Cu}\left(\mathrm{ClO}_{4}\right)_{2}\right)$ showed that $\mathrm{Cu}_{3}(\mathrm{btc})_{2}$ displays similar or lower reaction rates but product selectivity were at least comparable. ${ }^{123}$

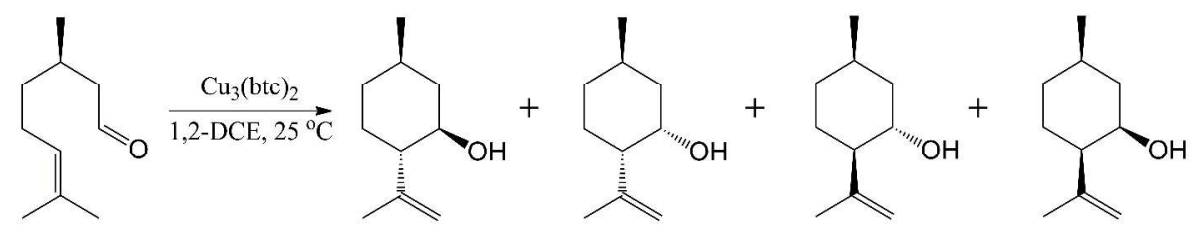

(+)-citroncllal $(-)$-isopulcgol

$(+)$-nco-isopulcgol

$(+)$-iso-isopulcgol $\quad(+)$-ncoiso-isopulcgol

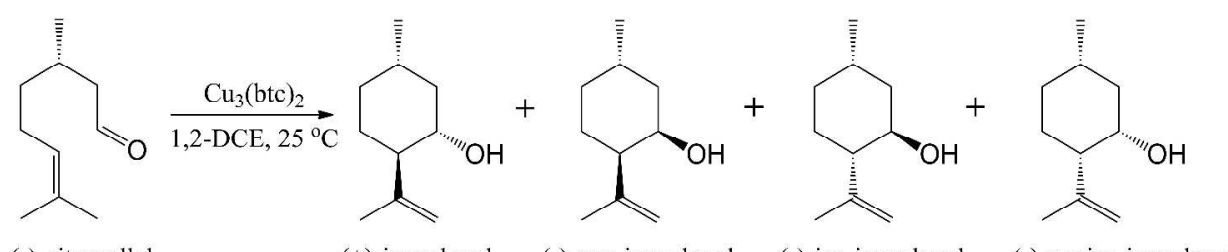

(-)-citronellal

$(+)$-isopulegol

(-)-neo-isopulegol

(-)-iso-isopulegol

(-)-neoiso-isopulegol

Scheme 27. Cyclization of racemic citronellal to pulegol isomers.

Jeong and coworkers studied the cyclization of 3-methylgeranial leading to the cyclic terpenoid analogue (Scheme 28) through the Lewis-acid-promoted carbonyl-ene reaction over chiral MOF $\mathrm{Cu}_{2}\left((\mathrm{~S})-2,2^{\prime} \text {-dihydroxy-6,6'-dimethyl(1,1'-biphenyl)-4,4'-dicarboxylate }\right)_{2}\left(\mathrm{H}_{2} \mathrm{O}\right)_{2}$ modified by methylation of free hydroxy groups in the linker using dimethylzinc. ${ }^{109}$ Only a single diastereomer was obtained at $0{ }^{\circ} \mathrm{C}$ with $23 \%$ ee at $89 \%$ yield using 1.5 eq of $\mathrm{Zn}$ modified MOF. ${ }^{109}$ With 3 eq. of MOF, the reaction proceeded with a considerably higher chemical yield (92\%) as well as a higher stereoselectivity $\left(50 \%\right.$ ee). ${ }^{109}$ Dimethylzinc-modified or non-modified MOF did not promote the reaction. It was noticed that after complete disappearance of 3-methylgeranial, no product was observed, indicating strong coordination of the product to the active sites in Zn-modified MOF. ${ }^{109}$ The desired product was observed only after destruction of the MOF crystals and liberation of the product. It should be noted, that the overall reaction rate for the heterogeneous MOF was comparable to the homogeneous reaction by Zn-BINOL complex. ${ }^{109}$ 


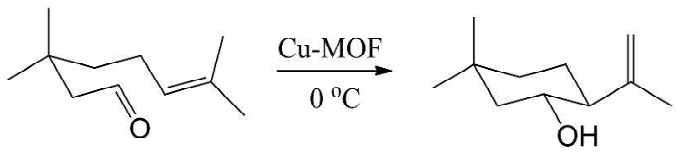

Scheme 28. Cyclization of 3-methylgeranial leading to the cyclic terpenoid.

Opanasenko et al. investigated the catalytic performance of a set of $\mathrm{MOFs}\left(\mathrm{Cu}_{3}(\mathrm{btc})_{2}\right.$, Fe(btc), MIL-100(Fe), MIL-100(Cr), ZIF-8, MIL-53(Al)) for the Prins condensation of $\beta$ pinene with formaldehyde (Scheme 29) and compared it with the catalytic behaviour of conventional zeolites BEA and USY and titanosilicate zeolite MFI (TS-1). ${ }^{124}$ The activity of investigated MOFs increased upon increase in the concentration of accessible Lewis acid sites in the order ZIF-8 (3\% yield at $80{ }^{\circ} \mathrm{C}$ with p-xylene as solvent $)<$ MIL-53(Al) $(44 \%)<\mathrm{Fe}(\mathrm{btc})$ $(51 \%)<$ MIL-100(Cr) $(65 \%)<$ MIL-100(Fe) $(87 \%) .{ }^{124}$ In contrast to zeolites, $\beta$-pinene isomerization, undesirable side reaction, did not take place on the relatively mild acid sites of MOFs, which showed significantly higher selectivity to the target nopol in comparison with zeolites. $^{124}$ The yield of nopol in the presence of MIL-100(Fe) as catalyst increased with the increase of the relative solvent polarity in the order: dodecane $\left(51 \%\right.$ yield at $80{ }^{\circ} \mathrm{C}$ with 0 polarity $)<$ p-xylene $(64 \%, 0.074$ polarity $)<$ cyclohexanone $(68 \%, 0.281$ polarity $)<$ acetonitrile ( $82 \%, 0.46$ polarity). ${ }^{124}$ Preservation of the structure and active sites of MIL-100 $(\mathrm{Fe})$, and the possibility to use the catalyst at least in three catalytic cycles without loss of activity was shown. ${ }^{124}$

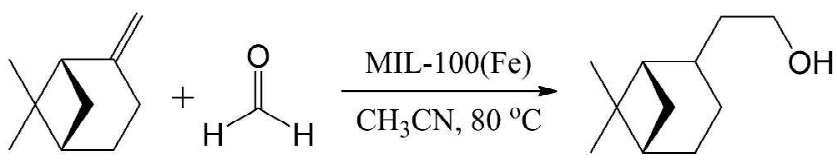

Scheme 29. Prins condensation of $\beta$-pinene with formaldehyde to nopol.

\section{Synthesis of fine chemicals through oxidation reactions}


MOFs are solid materials containing a high density of transition metals with various possible oxidation states, many of them being active to promote different types of oxidation when they are in solution. For this reason, MOFs appear in principle as suitable solid catalysts for oxidations. The reported catalytic activity of MOFs for oxidation of organic compounds has been reviewed, ${ }^{154}$ but considering that the field is progressing quickly we update the use of MOFs as oxidation catalysts for the production of fine chemicals. It has been found that MOFs can promote oxidation of cycloalkanes, ${ }^{155}$ alkenes ${ }^{156}$ and alcohols. ${ }^{157}$ In the cases of the aerobic oxidation promoted by MOFs, a co-catalyst such as TEMPO or N-hydroxyphthalimide is generally adsorbed within MOF. ${ }^{155}$ According to the general comments above, MOFs could have an improved performance compared to other micro-/mesoporous materials for liquid phase reactions under conditions that are compatible with the structural integrity of the MOF and allow its recovery and reuse. The advantages of MOFs with respect to other porous materials for liquid phase reactions in the production of fine chemicals are also valid for oxidation reactions, with the additional feature that MOFs have a high content in transition metals that are the conventional type of oxidation site. Specifically, zeolites and mesoporous aluminosilicates are devoid of activity for oxidation reactions. However, the presence of transition metals grafted on the framework may introduce activity for oxidation reactions in (alumino)silicates. ${ }^{158,159}$ Other microporous materials like AlPOs can also be modified to introduce catalytic activity. ${ }^{160}$ However, these grafted metals tend to leach out of the (alumino)silicate or aluminophosphate framework, ${ }^{159,}{ }^{161}$ particularly in liquid phase reactions, the lack of stability together with diffusion restrictions being the two major limitations of conventional porous solids.

The present section is focused on the use of MOFs as oxidation catalysts in the synthesis of fine chemicals. As we will comment later, this area is certainly under developed and more examples of application of MOFs as oxidation catalysts will appear in the next years. 
MOFs as catalysts or supports have been widely tested for the epoxidation of cyclohexene, styrene and cyclopentene. ${ }^{154}$ In most of the examples described in the literature, tert-butylhydroperoxide (TBHP) was used as oxidant. Thus, $\mathrm{Co}_{4}{ }_{4} \mathrm{O}(\mathrm{bdpb})_{3}\left(\mathrm{H}_{2} \mathrm{bdpb}\right.$ : 1,4bis $[(3,5$-dimethyl $)$ pyrazol-4-yl $]$ benzene $), \mathrm{NH}_{4}\left[\mathrm{Cu}_{3}-\left(\mu_{3}-\mathrm{OH}\right)\left(\mu_{3}-4 \text {-carboxypyrazolato }\right)_{3}\right]$ anionic MOF, $\left[\mathrm{Cu}\left(\mathrm{H}_{2} \text { btec }\right)(\text { bipy })\right]_{\propto}\left(\mathrm{H}_{4} \text { btec: } 1,2,4,5 \text {-benzenetetracarboxylic acid }\right)^{162}$ and MIL-47(V) are active to promote cyclohexene oxidation using TBHP as oxidant, but these studies have not been expanded towards more complex substrates and they are out of the scope of this review. ${ }^{163-166}$ Other MOF, such as $\mathrm{Pd}^{\mathrm{II}}$-porphyrin-based MOF with the molecular formula of $\left[\mathrm{Cd}_{1.25}\left(\mathrm{Pd}-\mathrm{H}_{1.5} \mathrm{tcpp}\right)\left(\mathrm{H}_{2} \mathrm{O}\right)\right] .2 \mathrm{DMF} \quad\left(\mathrm{H}_{4} \mathrm{tcpp}: \quad\right.$ 5,10,15,20-tetra(carboxyphenyl)palladiumporphyrin), showed significant catalytic activity for styrene oxidation to acetophenone and benzaldehyde using hydrogen peroxide as oxidant in perchloric acid. ${ }^{167}$

While TBHP or $\mathrm{H}_{2} \mathrm{O}_{2}$ are considered green oxidants and tert-butyl alcohol or water are the by-products of these reactions, the general tendency is to replace TBHP by oxygen from the view point of sustainability and cost of the oxidant. In this regard, post-functionalization of $\mathrm{NH}_{2}-\mathrm{MIL}-47(\mathrm{~V})$ with $\mathrm{TiO}(\mathrm{acac})_{2}$ to create a bimetallic (V and $\mathrm{Ti}$ ) oxidation catalyst, namely $\mathrm{NH}_{2}-\mathrm{MIL}-47$ Ti], has been reported to promote cyclohexene oxidation using oxygen as oxidant and cyclohexanecarbaldehyde as cocatalyst. ${ }^{168}$ Similarly, a manganese(II) acetylacetonate complex immobilized into the amino functionalized isoreticular metal-organic framework IRMOF-3 has been reported for the epoxidation of cyclohexene, cyclooctene and styrene using pivalaldehyde and oxygen as oxidant. ${ }^{169}$ Epoxidation of linear and cyclic olefins has been reported using two lanthanide-based three-directional MOFs namely $\left[\mathrm{Nd}(\mathrm{HCOO})_{3}\right]_{\mathrm{n}}$ and $\left[\mathrm{Pr}(\mathrm{HCOO})_{3}\right]_{\mathrm{n}}$ as catalysts. ${ }^{170}$

Table 2 summarizes various catalytic methods reported on the synthesis of fine chemicals through oxidation reactions and reduction (hydrogenations) using MOFs as catalysts. 
Table 2. MOF-based solid catalysts employed in oxidations with the corresponding oxidizing agent

\begin{tabular}{|c|c|c|c|}
\hline MOF formula & Reaction & Oxidant & Ref \\
\hline $\mathrm{Zn}_{2}(\mathrm{bpdc})_{2} \mathrm{~L}^{\mathrm{a}} .10 \mathrm{DMF} .8 \mathrm{H}_{2} \mathrm{O}$ & $\begin{array}{l}\text { epoxidation of 2,2-dimethyl-2H- } \\
\text { chromene }\end{array}$ & $\begin{array}{l}\text { Iodosyl-2-(t- } \\
\text { butylsulfonyl) } \\
\text { benzene }\end{array}$ & 171 \\
\hline Co-POM/MIL-101 & $\begin{array}{l}\text { oxidation of } \alpha \text {-pinene, } \\
\text { caryophyllene and cyclohexene }\end{array}$ & $\mathrm{O}_{2}$ or $\mathrm{H}_{2} \mathrm{O}_{2}$ & 172 \\
\hline $\mathrm{PW}_{12} / \mathrm{MIL}-101$ & 3 -carene, $\alpha$-pinene and limonene & $\mathrm{H}_{2} \mathrm{O}_{2}$ & 173 \\
\hline PW $11 @ M I L-101(C r)$ & $\begin{array}{l}\text { oxidation of cis-cyclooctene and } \\
\text { geraniol }\end{array}$ & $\mathrm{H}_{2} \mathrm{O}_{2}$ & 174 \\
\hline Sc-MOF & oxidation of linalool & $\mathrm{H}_{2} \mathrm{O}_{2}$ & 175 \\
\hline MIL-101(Cr) & $\begin{array}{l}\text { oxidation of cyclohexene, } \\
\text { limonene, } \alpha \text {-pinene }\end{array}$ & TBHP in decane & 176 \\
\hline $\begin{array}{l}\text { MIL-101(Fe) and MIL- } \\
101(\mathrm{Cr})\end{array}$ & $\begin{array}{l}\text { oxidation of cyclohexene and } \alpha \text { - } \\
\text { pinene }\end{array}$ & $\mathrm{O}_{2}$ & 177 \\
\hline Fe/MIL-101(Cr) & $\begin{array}{l}\text { oxidation of styrene and } \\
\text { cyclohexene }\end{array}$ & $\mathrm{H}_{2} \mathrm{O}_{2}$ & 178 \\
\hline MIL-101(Cr) & $\begin{array}{l}\text { oxidation of cyclopentene and } \\
\text { cyclohexene }\end{array}$ & $\mathrm{H}_{2} \mathrm{O}_{2}$ & 179 \\
\hline $\begin{array}{l}{\left[\mathrm{Al}(\mathrm{bpydc})(\mathrm{OH}) \cdot 0.5 \mathrm{H}_{2} \mathrm{O}\right]} \\
(\mathrm{MOF}-253)\end{array}$ & oxidation of cyclohexane & $\mathrm{O}_{2}$ & 180 \\
\hline $\begin{array}{l}\text { MIL-101(Fe) and MIL- } \\
101(\mathrm{Cr})\end{array}$ & oxidation of cyclohexane & TBHP and $\mathrm{O}_{2}$ & 181 \\
\hline$\left\{\left[\mathrm{Cd}\left(\mathrm{L}^{\mathrm{b}}\right)\left(\mathrm{H}_{2} \mathrm{O}\right)\right] \cdot 3 \mathrm{H}_{2} \mathrm{O}\right\} \infty$ & oxidation of ethylbenzene & TBHP & 182 \\
\hline $\begin{array}{l}{\left[\mathrm{Mn}_{5} \mathrm{Cl}_{2}(\mathrm{MnCl}-\mathrm{OCPP})-\right.} \\
\left.(\mathrm{DMF})_{4}\left(\mathrm{H}_{2} \mathrm{O}\right)_{4}\right] \cdot 2 \mathrm{DMF} \cdot 8 \mathrm{CH} \\
{ }_{3} \mathrm{COOH} \cdot 14 \mathrm{H}_{2} \mathrm{O}(\mathrm{ZJU}-18), \\
{\left[\mathrm{Mn}_{5} \mathrm{Cl}_{2}(\mathrm{Ni}-\right.} \\
\left.\mathrm{OCPP})\left(\mathrm{H}_{2} \mathrm{O}\right)_{8}\right] \cdot 7 \mathrm{DMF}- \\
\cdot 6 \mathrm{CH}_{3} \mathrm{COOH} \cdot 11 \mathrm{H}_{2} \mathrm{O}(\mathrm{ZJU}- \\
19) \text {, and }\left[\mathrm{Cd}_{5} \mathrm{Cl}_{2}(\mathrm{MnCl}-\right.\end{array}$ & oxidation of ethylbenzene & TBHP & 183 \\
\hline
\end{tabular}




\begin{tabular}{|l|l|c|c|}
\hline $\begin{array}{l}\text { OCPP)- } \\
\left.\left(\mathrm{H}_{2} \mathrm{O}\right)_{6}\right] \cdot 13 \mathrm{DMF} \cdot 2 \mathrm{CH}_{3} \mathrm{COO} \\
\mathrm{H} \cdot 9 \mathrm{H}_{2} \mathrm{O}(\mathrm{ZJU}-20)\end{array}$ & & & \\
\hline $\mathrm{Au}-\mathrm{Pd} / \mathrm{MIL}-101(\mathrm{Cr})$ & oxidation of toulene & $\mathrm{O}_{2}$ & 184 \\
\hline MOF-253-Ru7 & $\begin{array}{l}\text { oxidation of benzyl, cyclic } \\
\text { alcohols }\end{array}$ & $\mathrm{PhI}(\mathrm{OAc})_{2}$ & 185 \\
\hline Fe/ MIL-101(Cr) & oxidation of benzyl alcohols & $\mathrm{H}_{2} \mathrm{O}_{2}$ & 178 \\
\hline
\end{tabular}

${ }^{\mathrm{a}} \mathrm{L}: \quad$ (R,R)-(-)-1,2-cyclohexanediamino-N,N'-bis(3-tert-butyl-5-(4-pyridyl)salicylidene)Mn $\left.{ }^{\mathrm{III}} \mathrm{Cl}\right)$; ${ }^{\mathrm{b}} \mathrm{L}=4,4$ '-(9,10-anthracenediyl)dibenzoic acid).

This section aims to describe the use of MOFs as oxidation catalysts in three parts. The first part summarizes the use of MOFs as oxidation catalyst in the oxidation of alkenes including epoxidation/allylic oxidation and $\mathrm{C}=\mathrm{C}$ double bond cleavage. The second part will focus on the oxidation of $\mathrm{C}-\mathrm{H}$ bonds in cyclic and benzylic hydrocarbons. The final part deals with the results obtained on oxidation of alcohols by MOFs as catalysts. In all these parts, efforts are made to highlight the significance of the results and catalyst stability. Also, this section restricts to those papers appeared after 2011 complementing a review published from our group in this field.(ref)

\section{Oxidation of alkenes}

\section{Epoxidation/allylic oxidation}

One of earlier studies on enantioselective epoxidation has been reported by Cho and coworkers using $\mathrm{Zn}_{2}(\mathrm{bpdc})_{2} \mathrm{~L}$.10DMF. $8 \mathrm{H}_{2} \mathrm{O}$ (where $\mathrm{L}$ is a Mn-Schiff base complex) as catalyst for 2,2-dimethyl-2H-chromene with iodosyl-2-(tert-butylsulfonyl)benzene as oxidant (Scheme 30). ${ }^{171}$ Initially, the Mn complex analog not bonded to the MOF structure exhibited slightly higher reactivity than the MOF solid having the Mn complex integrated on the struts, but after a 
few hours the ligand loosed its activity. ${ }^{171}$ For this reason, at final reaction time the catalyst showed four times higher reactivity than the Mn complex confirming the superior performance of MOF as catalyst, achieving $82 \%$ enantioselectivity in the final epoxide. While trying to reuse the catalyst, the TON decreased from 1430 to 1320 from $1^{\text {st }}$ to $3^{\text {rd }}$ run, respectively, without affecting the enantioselectivity. This partial decrease in the catalytic activity was due to the loss between 4 and $7 \%$ of Mn present initially in the framework. ${ }^{171}$ Although the percentage of Mn leaching is low, for certain uses it could still be a limitation overcoming the maximum tolerable impurity metal level in the final product. ${ }^{186}$ In any case, the higher activity of the Mn-salen complex attached to MOF with respect to the analogous in solution and the possibility to reuse by simply recovery of the solid, nicely exemplifies the advantages of grafting the active site in a tridimensional, highly porous structure characteristic of MOFs.

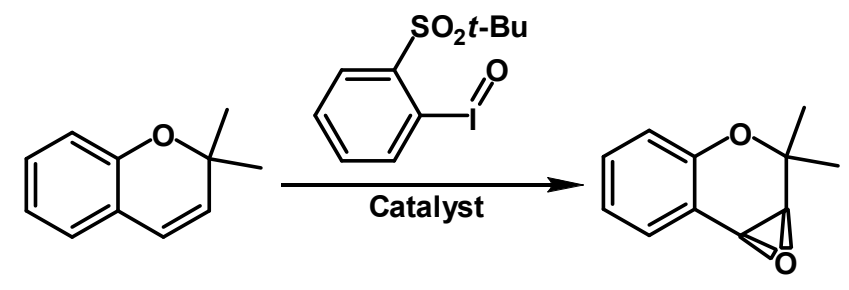

Scheme 30. Enantioselective epoxidation catalyzed by $\mathrm{Zn}_{2}(\text { bpdc })_{2}$ L.10DMF. $8 \mathrm{H}_{2} \mathrm{O}$.

Titanium- and cobalt-monosubstituted Keggin heteropolyanions, $\left[\mathrm{PW}_{11} \mathrm{CoO}_{39}\right]^{5-}$ and $\left[\mathrm{PW}_{11} \mathrm{TiO}_{40}\right]^{5-}$ have been bound to MIL-101 by means of electrostatic attraction and used as catalyst for the oxidation of $\alpha$-pinene, caryophyllene and cyclohexene using hydrogen peroxide and oxygen as oxidants (Scheme 31). ${ }^{172}$ Oxidation of cyclohexene with hydrogen peroxide over Ti-POM/MIL-101 results exclusively in allylic oxidation products namely 2-cyclohexenol and 2-cyclohexenone with no evidence for $\mathrm{C}=\mathrm{C}$ epoxidation. ${ }^{172}$ It is important to note that although, Ti-POM and MIL-101 produced low conversion of cyclohexene using hydrogen peroxide, TiPOM/MIL-101 showed $39 \%$ conversion resulting in $32 \%$ of 2-cyclohexenol and 2cyclohexenone, $46 \%$ of 1,2-dihydroxycyclohexane. ${ }^{172}$ Similarly to cyclohexene, oxidation of 
$\alpha$-pinene led to the formation of verbenol and verbenone whereas the epoxide was not observed in any case. ${ }^{172}$ Using oxygen as oxidant, Co-POM/MIL-101 resulted in $45 \%$ conversion of $\alpha$ pinene with $29 \%$ of verbenol and $27 \%$ of verbenone in $2 \mathrm{~h}$. On the other hand, Ti-POM/MIL101 showed $40 \%$ conversion with $32 \%$ of verbenol and $30 \%$ of verbenone in $2 \mathrm{~h}^{172}$ These catalysts showed no evidence of leaching according to the hot filtration test and the elemental analysis of POM suggesting the stability of catalyst. ${ }^{172}$ Further, Co-POM/MIL-101 can be reused for five times with almost similar activity as that of fresh catalyst with oxygen as oxidant. ${ }^{172}$ Powder XRD of the reused catalyst showed no significant changes in the crystalline structure compared to the fresh catalyst. ${ }^{172}$ However, nitrogen adsorption studies revealed a 20 $\%$ decrease in the specific surface area and pore volume, which most likely can be explained by adsorption of byproducts. ${ }^{172}$ In contrast, caryophyllene exhibited $88 \%$ conversion with $100 \%$ selectivity to caryophyllene-4,5-monoepoxide using Ti-POM/MIL-101 with $0.2 \mathrm{M}$ of hydrogen peroxide. ${ }^{172}$ Importantly, the selectivity of caryophyllene epoxidation was significantly higher for Ti-POM/MIL-101 than for homogeneous Ti-POM (60 \% conversion with $52 \%$ selectivity). ${ }^{172}$ It has been proposed that this higher epoxide selectivity of Ti-POM/MIL-101 derives from the fact that deprotonation of the Ti-POM anion on immobilization within the MIL-101 cages via anion exchange reduces the percentage of secondary products arising from the acid catalyzed ring opening of the epoxide. ${ }^{172}$ The observed different activity of the catalyst for cyclohexene and $\alpha$-pinene may be due to the harsh conditions employed for cyclohexene like excess hydrogen peroxide $(0.4 \mathrm{M})$ and high temperature $\left(70{ }^{\circ} \mathrm{C}\right)$ compared to $\alpha$-pinene oxidation $\left(0.14 \mathrm{M} \mathrm{H}_{2} \mathrm{O}_{2}, 30{ }^{\circ} \mathrm{C}\right) .{ }^{172}$ FT-IR spectroscopy, small-angle XRD, and $\mathrm{N}_{2}$ adsorption have shown collectively that MIL-101 was not stable at $0.4 \mathrm{M}$ hydrogen peroxide concentration which was employed for $\mathrm{C}=\mathrm{C}$ oxidation. After three consecutive treatments of MIL-101 with $0.1 \mathrm{M}$ of hydrogen peroxide at $70{ }^{\circ} \mathrm{C}$, no powder XRD was observed. It becomes highly 
relevant to optimize the reaction conditions to preserve the MOF structure and also to avoid further side reactions in order to favour selectively epoxide formation.
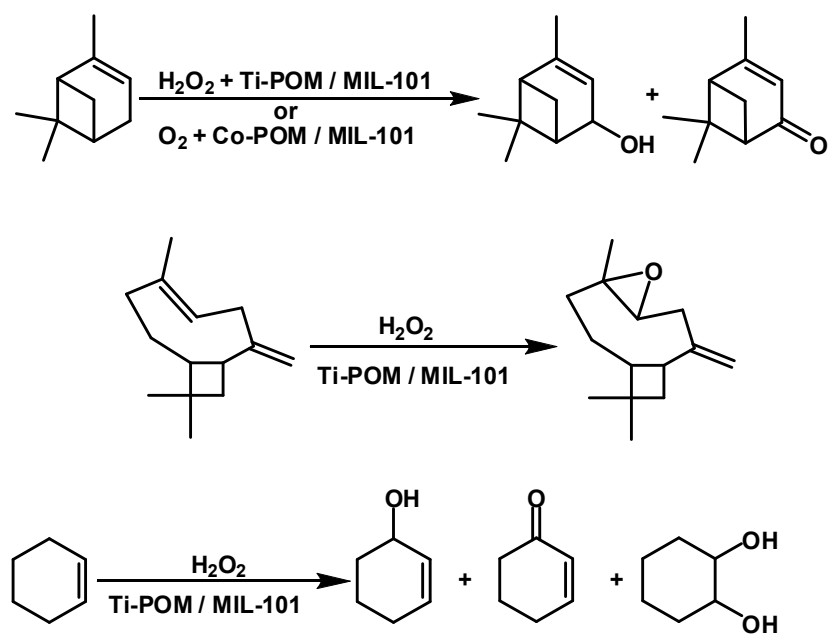

Scheme 31. Oxidation reactions catalyzed by metal containing POM supported on MIL-101.

Polyoxotungstates $\mathrm{PW}_{4}$ and $\mathrm{PW}_{12}$ have been inserted electrostatically into the nanocages of MIL-101 at a loading between 5 and 14 wt $\%$ and used as catalyst for $\mathrm{C}=\mathrm{C}$ oxidation. ${ }^{173}$ Using cyclohexene as model substrate and working with 2 eq. of $\mathrm{H}_{2} \mathrm{O}_{2}, 5 \%$ $\mathrm{PW}_{4} / \mathrm{MIL}-101$ showed $76 \%$ substrate conversion after $3 \mathrm{~h}$ with $74 \%$ selectivity of cyclohexene oxide. ${ }^{173}$ Very similar results in terms of conversion and cyclohexene oxide selectivity were achieved for $5 \% \mathrm{PW}_{12} / \mathrm{MIL}-101 .{ }^{173}$ The allylic oxidation products, 2cyclohexenol and 2-cyclohexenone (totally ca. 10\%) were identified as the main byproducts, while the product of epoxide ring-opening, 1,2-trans-cyclohexanediol, was not found in the reaction mixture. ${ }^{173}$ This contrasts with the homogeneously catalyzed oxidation in the presence of $\mathrm{PW}_{4}$ or $\mathrm{PW}_{12}$ where the diol was the main byproduct. Elemental analysis and hot filtration test showed the absence of any tungsten (from the polyoxotungstates) or chromium (from MIL101) traces in the liquid phase and that the reaction stops when the solid is removed. ${ }^{173}$ $\mathrm{PW}_{4} / \mathrm{MIL}-101$ showed a slight decrease in the epoxide yield after three runs and this could be 
attributed to the decrease in the specific surface area and pore volume. ${ }^{173}$ Powder XRD shows amorphization of the MIL-101 catalyst due to its partial decomposition during the cyclohexene oxidation with hydrogen peroxide. ${ }^{173}$

The basic study with cyclohexene has been expanded towards more relevant products for fine chemicals, 3-carene and limonene (Scheme 32). ${ }^{173}$ These two monoterpenes exhibited 96 and $89 \%$ of epoxide selectivity, respectively, using $5 \% \mathrm{PW}_{12} / \mathrm{MIL}-101$ with 2 eq. of $\mathrm{H}_{2} \mathrm{O}_{2}$ while $\mathrm{PW}_{12}$ showed 78 and $65 \%$ of epoxide selectivity, respectively. ${ }^{173}$ Limonene was oxidized to limonene 1,2-epoxide. ${ }^{173}$ This remarkable performance of the $\mathrm{PW}_{12} / \mathrm{MIL}-101$ hybrid has been attributed to the ability of MIL-101 to adsorb preferably $\mathrm{H}_{2} \mathrm{O}_{2}$ and alkene with respect to water, due to the hydrophobicity of the organic linker while $\mathrm{PW}_{12}$ adsorbs much higher amount of water. ${ }^{173}$ In contrast, $\alpha$-pinene exhibited $71 \%$ of epoxide selectivity using 5 $\% \mathrm{PW}_{12} / \mathrm{MIL}-101$ with 2 eq. of $\mathrm{H}_{2} \mathrm{O}_{2}$ (Scheme 32). ${ }^{173}$ This lower selectivity of $\alpha$-pinene epoxidation is a reflection of the influence of the substrate structure determining the reactivity of the $\mathrm{C}=\mathrm{C}$ double bond and diffusion of the substrate through the MOF pores.
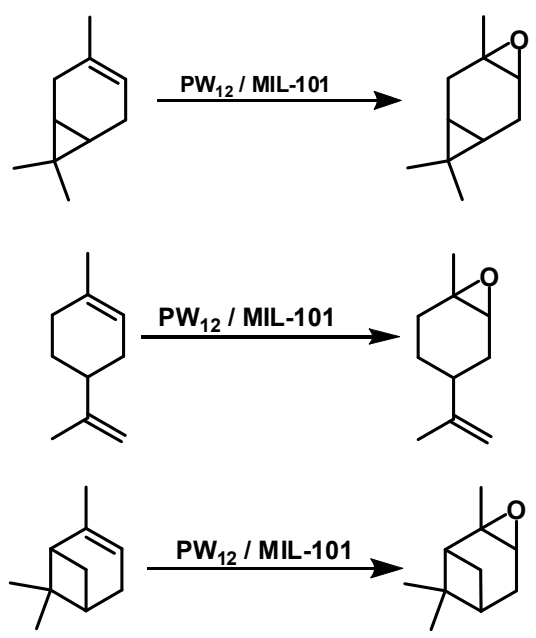

Scheme 32. $\mathrm{PW}_{12} / \mathrm{MIL}-101$ as catalyst for oxidation reactions.

PW $11 @$ MIL-101(Cr) and SiW $11 @$ MIL-101(Cr) were prepared by the inclusion of the potassium salts of the monovacant polyoxotungstates, $\left[\mathrm{PW}_{11} \mathrm{O}_{39}\right]^{7-}\left(\mathrm{PW}_{11}\right)$ and $\left[\mathrm{SiW}_{11} \mathrm{O}_{39}\right]^{8^{-}}$ 
$\left(\mathrm{SiW}_{11}\right)$ into MIL-101(Cr). ${ }^{174}$ The catalytic activities of these materials were tested in the oxidation of cis-cyclooctene and geraniol using the $\mathrm{H}_{2} \mathrm{O}_{2}$ as oxidant (Scheme 33). PW 11 @MIL$101(\mathrm{Cr})$ oxidized cis-cyclooctene into 1,2-epoxycyclooctane completely after 10 min $98 \%$ yield and TOF of 1922. Using an equivalent amount of homogeneous catalyst $\mathrm{PW}_{11}$ the conversion of cis-cyclooctene after 10 min was negligible but, however, after 5 h $96 \%$ yield of 1,2-epoxycyclooctane was achieved. The observed results clearly showed that the phosphotungstate catalysts $\mathrm{PW}_{11}$ and $\mathrm{PW}_{11} @$ MIL-101(Cr) seemed to be more active than the analogues silicotungstates. This was believed to be due to the weaker interactions between $\mathrm{H}_{2} \mathrm{O}_{2}$ and silicotungstates than with phosphotungstates. ${ }^{187}$ On the other hand, $\mathrm{SiW}_{11} @$ MIL101(Cr) showed complete conversion of cis-cyclooctene after 6 h with $93 \%$ yield, but in the presence of the homogeneous $\mathrm{SiW}_{11}$ catalyst only $53 \%$ of conversion of cis-cyclooctene was observed. These results show that the inclusion of the active centres $\mathrm{PW}_{11}$ and $\mathrm{SiW}_{11}$ seems to improve the catalytic performance of these catalysts. This behavior may be attributed to concentration effects promoted by adsorption of reactants in the cages of MIL-101(Cr). It may also be that the improved catalytic performance of the polyoxometallates incorporated inside MOF derives from stabilization of the catalytic sites against decomposition and aggregation that usually constitute important deactivation pathways in molecular catalysts. Further studies comparing initial reaction rates and long term stabillity between the homogeneous and heterogeneous congeners will clarify this issue.

In the present case, homogeneous and heterogeneous catalysts give rise to 2,3epoxygeraniol as product, exhibiting a high chemoselectivity. The silicotungstates $\mathrm{SiW}_{11}$ and SiW $11 @$ MIL-101(Cr) were more active than the respective phosphotungstates PW $_{11}$ and PW $11 @$ MIL-101(Cr). The kinetic profiles of SiW H1 $_{11}$ and SiW $11 @$ MIL-101(Cr) are similar and the conversion of geraniol into 2,3-epoxygeraniol was almost complete after $1 \mathrm{~h}$. In contrast, the catalytic activity of $\mathrm{PW}_{11} @$ MIL-101(Cr) is considerably lower than that of the 
corresponding homogeneous catalyst $\mathrm{PW}_{11}$. This contrasting behavior in the oxidation of geraniol compared to cis-cyclooctene may be derived from the different coordination of the substrate to the catalyst for $\mathrm{PW}_{11}$ and $\mathrm{SiW}_{11}$. Oxidation of allylic alcohols in polyoxometallates is known to involve coordination of the hydroxyl group to the polyoxometallate and the formation of a peroxo group adjacent to this site. ${ }^{188}$ In general, the activity of PW $_{11} @$ MIL101(Cr) and SiW $11 @$ MIL-101(Cr) in the oxidation of cis-cyclooctene and geraniol slightly decreases from the first to the second cycle during the first few hours. However after $24 \mathrm{~h}$, the conversion is the same for three cycles corresponding to the complete conversion of substrate. From the second to the third reaction cycle the activity of the catalysts is maintained. This slight decrease in the activity may indicate the occurrence of some leaching of the active species $\left(\mathrm{PW}_{11}\right.$ or $\left.\mathrm{SiW}_{11}\right)$ during the first reaction cycle. It was found that the leaching of $\mathrm{PW}_{11}$ species from $\mathrm{PW}_{11} @$ MIL-101(Cr) during the reaction cycles was 17\% of the initial loading. With regard to the stability of the MIL-101(Cr) framework, powder XRD patterns of the two heterogeneous catalysts are identical to the respective diffractogram before catalytic use, confirming the integrity of the crystalline structure of MIL-101(Cr) after three cycles.

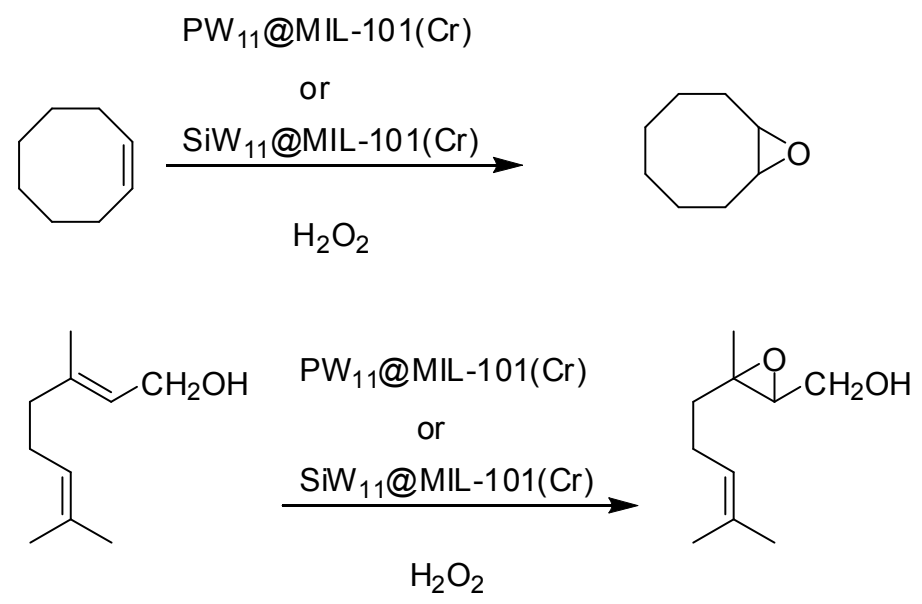

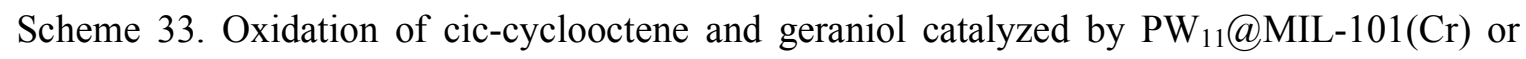
SiW11@MIL-101(Cr). 
3,7-Dimethylocta-1,6-dien-3-ol (linalool) is the precursor of various oxides commonly found in nature that are mainly used in the perfumery industry as fragrances. Furthermore, they seem to play a critical biological role in several pollination systems acting as insect attractants. ${ }^{189}$ Given the importance of linalool oxide, different MOFs with Sc and Y were synthesized with 1,5-and 2,6-naphthalenedisulfonates as ligands, testing these MOFs as bifunctional, heterogeneous catalysts in the epoxidation of linalool (Scheme 34). ${ }^{175}$ Linalool was oxidized to pyranoid and furanoid ethers, promoted by Sc-based MOFs exhibiting a dual redox and acid activity, with a conversion of more than $60 \% .{ }^{175}$ However, in the case of Y as central metal atom with 1,5-naphthalenedisulfonates as ligand, the conversion of linalool under the same conditions was $100 \%{ }^{175}$ Further, it was noticed that the small size of the pores in these structures prevent the access of the substrate to the metallic centers and thus the catalytic reaction takes place only on the external surface of the particles. ${ }^{175}$ In addition, this situation is also inconvenient while considering the size of the substrate and intermediates to diffuse through the pores of MOFs. ${ }^{175}$ It was determined that these catalysts maintain the structural integrity and no leaching of active sites was observed. ${ }^{175}$

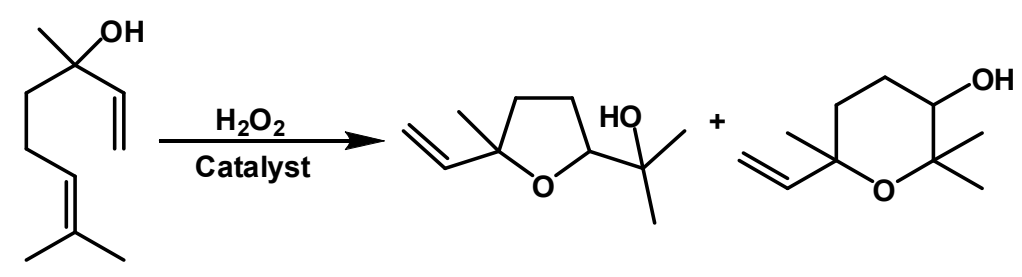

Scheme 34. Oxidation of linalool to furanoic and pyranoic structures.

In another precedent, the selective oxidation of cyclohexene to 2-cyclohexenone in benzene at $50{ }^{\circ} \mathrm{C}$ using MIL-101(Cr) has been reported, the catalyst pre-treatment playing a pivotal role in determining its activity. ${ }^{176}$ Under the best conditions, $92 \%$ of cyclohexene conversion was observed with $88 \%$ selectivity to 2-cyclohexenone and no epoxide was reported. ${ }^{176}$ The hot filtration test showed no leaching of active sites and powder XRD indicated no changes in the crystallinity before and after catalysis. ${ }^{176}$ The catalyst can be reused 
five times without any change in conversion and selectivity. A similar kind of reactivity was noticed with $\alpha$-pinene and limonene resulting in 87 and $70 \%$ conversion, respectively with the selectivity of the product being around $86 \%{ }^{176}$ Further detailed studies are required to rationalize the occurrence of selective allylic oxidation with no signs of alkene epoxidation.

MIL-101(Fe) and MIL-101(Cr) have been used as catalysts for the solvent-free oxidation of cyclohexene and $\alpha$-pinene with molecular oxygen. ${ }^{177}$ Both catalysts showed activity for alkene oxidation under mild conditions using 1 bar $\mathrm{O}_{2}$ with catalytic amount of TBHP as radical initiator at $40-60{ }^{\circ} \mathrm{C}$ to afford allylic oxidation products. The nature of catalysis and the product distribution strongly depends on the nature of the transition metal. MIL-101(Fe) resulted in $27 \%$ conversion of cyclohexene with 4, 63, 22 and $11 \%$ selectivity of cyclohexene oxide, cyclohexylhydroperoxide, 2-cyclohexenone and 2-cyclohexenol respectively at $50{ }^{\circ} \mathrm{C}$ in $16 \mathrm{~h} .{ }^{177}$ Under identical conditions, MIL-101(Cr) showed $12 \%$ conversion with 17,58 and $25 \%$ selectivity of cyclohexylhydroperoxide, 2-cyclohexenone and 2-cyclohexenol, respectively. ${ }^{177}$ The catalysis by MIL-101(Cr) was proved to be true heterogeneous at 40 and $60{ }^{\circ} \mathrm{C}$. In contrast, MIL-101(Fe) revealed a lower stability at $60{ }^{\circ} \mathrm{C}$ where some metal leaching $(7 \mathrm{ppm})$ is detected, although it behaves as true heterogeneous at 40 ${ }^{\circ} \mathrm{C}$. In this regard, powder XRD and $\mathrm{N}_{2}$ adsorption measurements clearly showed that the structure of MIL-101(Fe) partially collapsed after several reuses, although FT-IR spectroscopy still revealed the presence of the main characteristic bands of the MOF. It should be, however, commented that IR spectroscopy is not very informative regarding porosity and crystallinity. Nevertheless, under optimal conditions both $\mathrm{Cr}$ and Fe-catalysts could be recycled at least four times without loss of the catalytic activity. Since the reaction products inhibit the oxidation process due to their strong adsorption on the MOF active centers, alkene conversions cannot be high. 


\section{Oxidative cleavage of $\mathrm{C}=\mathrm{C}$ double bond}

Iron oxide $\left(\mathrm{Fe}_{2} \mathrm{O}_{3}\right)$ functionalized with MIL-101(Cr) was used catalyst for the oxidation of olefins in aqueous phase using hydrogen peroxide as oxidant. ${ }^{178}$ The iron content was measured by ICP/MS and found to be 2.06 wt.\%. Interestingly, the incorporation of Fe into MIL-101(Cr) rendered a material with a remarkably higher Lewis acidity. ${ }^{178}$ The catalytic activity of Fe/MIL-101(Cr) was tested in the oxidation of styrene to benzaldehyde (Scheme 35) using hydrogen peroxide as oxidant in water under microwave irradiation at $110-120{ }^{\circ} \mathrm{C}$. High yields were obtained for a variety of styrenes to their corresponding aldehydes. On the other hand, $92 \%$ cyclohexene was converted to 1,2-cyclohexanedione with $86 \%$ selectivity. Although, $20 \%$ of 1-hexene was oxidized, the corresponding aldehyde was not detected. It was believed that the high activity of Fe/MIL-101(Cr) comes from the incorporation of iron oxide nanoparticles by creating redox sites reponsible for the activity in redox reactions. ${ }^{178}$ Further, the high activity observed for these systems might correlate to changes on $\mathrm{Fe}$ electronic environment and oxidation state via interaction with the $\mathrm{Cr}$ in coordinatively unsaturated sites. Under the optimized reaction conditions, the catalyst was stable for three cycles and no iron was detected in the leaching experiment as evidenced by ICP-MS, thus, ruling out the possibility of some contrbution of homogeneous catalysis to the process. Powder XRD and nitrogen adsorption studies showed that the structural integrity was maintained during the catalytic studies.

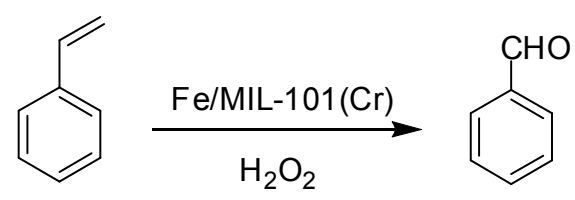

Scheme 35. Oxidative cleavage of styrene to benzaldehyde catalyzed by Fe/MIL-101(Cr).

Recently, MIL-101(Cr) has been reported to catalyze the oxidation of alkenes to their corresponding carboxylic acids using hydrogen peroxide as oxidant in acetonitrile under reflux 
conditions. ${ }^{179}$ Cyclic alkenes such as cyclopentene, cyclohexene, and cyclooctene were oxidized readily under the reaction conditions and resulted in yields of glutaric acid $(75 \%)$, adipic acid (90\%) and suberic acid (98\%), respectively. ${ }^{179}$ Even-numbered acyclic $\alpha$-olefins such as 1-octene, 1-decene, and 1-dodecene were easily oxidized into their corresponding oddnumbered alkanoic acids of one carbon less in around $80-90 \%$ yields. ${ }^{179}$ The substituted cyclic olefin, like indene, was oxidized to 2-(carboxymethyl)benzoic acid (homophthalic acid) in $100 \%$ yield. In order to probe the active sites responsible for this reaction, a control experiment was performed with $\mathrm{CrCl}_{3}$ in the oxidation of indene under the same conditions for MIL$101(\mathrm{Cr})$ and observed $17 \%$ of indene oxide. ${ }^{179}$ In contrast, $15 \%$ indene oxide was obtained in the absence of catalyst and no diacid was detected. ${ }^{179}$ Reusability was tested in the oxidation of indene and observed that the yield decreased gradually from 100, 94 and 81 for first, second and third cycle, respectively. This activity decrease was attributed to metal leaching and catalyst poisoning. Metal leaching determined by ICP analysis was found to be $18 \%$ of its initial content. Although powder XRD of the reused catalyst showed a similar pattern with low intensity diffraction peaks, nitrogen adsorption of reused catalyst showed a decrease in the surface area from 2125 to $1790 \mathrm{~m}^{2} / \mathrm{g}$. ${ }^{179}$ The use of hydrogen peroxide may be acceptable from green chemistry point of view, but it is always convenient determining its optimal concentration in order to reduce its consumption and to preserve the catalyst during the oxidation reaction as it contains water. In the present case, the reasons for the requirement of large excess of hydrogen peroxide $(5 \mathrm{mmol})$ to oxidize $0.5 \mathrm{mmol}$ of substrate are unclear.

\section{Oxidation of C-H bonds}

\section{Oxidation of cyclic hydrocarbons}

MOF-253 obtained from $\mathrm{Al}^{3+}$ and 2,2'-bipyridine-5,5'-dicarboxylic acid $\left(\mathrm{H}_{2}\right.$ bpydc) has been used as catalyst for the aerobic oxidation of cyclohexane. ${ }^{180}$ Under the 
optimized reaction conditions, MOF-253 showed $60.8 \%$ conversion with 47.9 and $31.3 \%$ of cyclohexanone and cyclohexanol selectivity respectively at $160{ }^{\circ} \mathrm{C}$ in $4 \mathrm{~h} .{ }^{180}$ The oxidation of cyclohexane clearly exhibited an initiation period of around $20 \mathrm{~min}$ in which almost no conversion was observed. Thereafter, the conversion increased gradually to reach $3 \%$ after $1 \mathrm{~h}$. However, the conversion of cyclohexane was greatly influenced by the reaction temperature as only $6.6 \%$ of oxidation products are observed at $120{ }^{\circ} \mathrm{C}$. ${ }^{180}$ Interestingly, $\mathrm{H}_{2}$ bpydc also exhibited $17.2 \%$ conversion of cyclohexane at $150{ }^{\circ} \mathrm{C}$ in $4 \mathrm{~h}$. Concerning the nature of the active sies it should be mentioned that the constituent metal ion (i.e. $\mathrm{Al}^{3+}$ ) in the MOF structure is fully coordinated and hence it does not have any free coordination position to promote the oxidation reaction. In order to rule out the contribution of $\mathrm{Al}^{3+}$, a small amount of $\mathrm{Al}(\mathrm{acac})_{3}$ was added with $\mathrm{H}_{2}$ bpydc in the reaction. ${ }^{180}$ It was observed that the introduction of $\mathrm{Al}^{3+}$ did not influence the conversion of cyclohexane. These results suggest that the open bpy moiety in MOF-253 might be the catalytic center for the oxidation transformation. To further confirm this finding, some other bpy derivatives like 2,2'-bipyridine and 1,10-phenanthroline were tested as catalysts and observed 8.4 and $13.8 \%$ conversion, respectively at $150{ }^{\circ} \mathrm{C}$. Hence, these experiments suggest the active participation of ligand in the aerobic oxidation. ${ }^{180}$ In contrast, the use of biphenyl or pyridine as the catalyst showed no products in the reaction. It was proposed that the bpy moieties might play a crucial role in the activation of molecular oxygen to form a superoxide radical anion, which further reacts with cyclohexane to produce cyclohexylhydroperoxide. ${ }^{180}$ To test this hypothesis, a mixture of MOF-253 in acetonitrile was preheated. After $0.5 \mathrm{~h}$, cyclohexane was injected to the mixture and observed no apparent induction period. The conversion increased rapidly to $30 \%$ (vs. $3 \%$ without the pre-activation process) after $60 \mathrm{~min}$. This result strongly support the role of MOF-253 linker in the initiation of the oxidation reaction via oxygen activation. It was also found that the MOF-253 has low band gap value between HOMO and LUMO of $3.98 \mathrm{eV}$ which facilitates the excitation of 
electrons from HOMO to LUMO, where the excited electrons could reduce molecular oxygen to form superoxide radical anion. This electronic excitation would require, however, photon absorption and will constitute a photochemical rather than a thermal process and should not take place in the dark. The catalyst was reused for five times with no appreciable changes in the conversion of cyclohexane and leaching of metal. ${ }^{180}$ This catalytic system was further extended to activate ethylbenzene, tetralin, diphenylmethane and cyclooctane with $46.8,68.9,38.3$ and $70.8 \%$ conversion, respectively. ${ }^{180}$ Toluene was exclusively oxidized to benzaldehyde with 10 $\%$ conversion. $^{180}$

In another precedent, MIL-101(Fe) and MIL-101(Cr) have been used as catalysts for the oxidation of cyclohexane using TBHP, $\mathrm{O}_{2}$ or their combination as oxidants under solvent free conditions. ${ }^{181}$ MIL-101(Cr) exhibited $36 \%$ of cyclohexane conversion with 8 and $75 \%$ selectivity of cyclohexanol and cyclohexanone, respectively, using $4 \mathrm{mmol}$ of TBHP at $70{ }^{\circ} \mathrm{C}$ in 8 h. ${ }^{181} \mathrm{~A}$ similar conversion was achieved under identical conditions, but with slightly higher selectivity of cyclohexanone $(81 \%)$ in the presence of argon. ${ }^{181}$ On the other hand, MIL$101(\mathrm{Cr})$ resulted in $9 \%$ conversion with 1 atm of $\mathrm{O}_{2}$ and $0.01 \mathrm{mmol}$ of TBHP. ${ }^{181} \mathrm{MIL}-101(\mathrm{Fe})$ exhibited $27 \%$ conversion of cyclohexane with $4 \mathrm{mmol}$ of TBHP. ${ }^{181}$ In the presence of MIL101(Fe), the main oxidation product was cyclohexylhydroperoxide. The cyclohexanol and cyclohexanone were formed in comparable yields with traces of over oxidation products. A similar result was obtained with $4 \mathrm{mmol}$ of TBHP indicating the role of molecular oxygen in the oxidation process. Unlike MIL-101(Cr), for cyclohexane oxidation with TBHP over MIL$101(\mathrm{Fe})$, the presence of air (oxygen) is essential. ${ }^{181}$ Under argon atmosphere, the conversion reached only $11 \%$ after $8 \mathrm{~h}$ (versus $27 \%$ in air) and cyclohexanone and cyclohexylhydroperoxide became the main oxidation products formed with 54 and $45 \%$ selectivity, respectively. In general, it was demonstrated that the cyclohexane oxidation can be performed over MIL-101(Cr) and MIL-101(Fe) using $\mathrm{O}_{2}$ and small additives of TBHP as 
initiator. In both cases, cyclohexylhydroperoxide was the main product, while cyclohexanol and cyclohexanone formed in similar amounts with the total selectivity to the primary oxidation products attained $94-99 \%$ at cyclohexane conversion of 9-11\% after 8 h. Both catalysts could be reused for five times and hot filtration experiments using MIL-101(Cr) showed negligible amount of chromium $(0.3 \mathrm{ppm}) .{ }^{181}$ It is interesting to note that this is lower compared to $\mathrm{Cr}$ PMO and Cr-TUD-1 as catalysts (9-34 ppm). ${ }^{190,191}$

\section{Oxidation of benzylic hydrocarbons}

A novel three-dimensional porous MOF with molecular formula, $\left\{\left[\mathrm{Cd}(\mathrm{L})\left(\mathrm{H}_{2} \mathrm{O}\right)\right] \cdot 3 \mathrm{H}_{2} \mathrm{O}\right\} \infty\left(\mathrm{L}=4,4^{\prime}-(9,10\right.$-anthracenediyl)dibenzoic acid) has been synthesized and de-solvated. The solvent-removed catalyst showed activity in the oxidation of ethylbenzene. ${ }^{182}$ This de-solvated framework exhibited permanent porosity (Langmuir surface area, $324 \mathrm{~m}^{2} / \mathrm{g}^{-1}$ ) with high thermal stability up to $380{ }^{\circ} \mathrm{C}$. Oxidation of ethylbenzene resulted in the trace amount of acetophenone in the absence of catalyst or in the presence of sodium perchlorate and hydrogen peroxide. The as-synthesized MOF showed $7.6 \%$ conversion with TBHP as oxidant in acetonitrile at $70{ }^{\circ} \mathrm{C}$ in $12 \mathrm{~h} .{ }^{182}$ Under identical conditions, the de-solvated MOF resulted in $92.6 \%$ of conversion with complete selectivity to acetophenone. ${ }^{182} 4-$ Ethylanisole and diphenylmethane were also oxidized to their corresponding ketones in nearly $100 \%$ yields with $100 \%$ selectivity. ${ }^{182}$ In the case of 4-(4-tert-butylphenyl) ethylbenzene with higher size of molecular dimension, 77.2 \% yield was obtained. This lower yield can be taken as evidence in support that the reaction takes place, at least in part, within the interior of the pores, where diffusion limitations can reduce product yiel of large molecules compared to smaller molecules. The catalyst was reused for three times and powder XRD showed that the crystal structure of the material was preserved during the reaction. ${ }^{182}$ Hot filtration experiment indicated $0.01 \mathrm{mg} / \mathrm{L}$ of $\mathrm{Cd}(\mathrm{II})$ in the solution and this result confirms the stability of catalyst 
under the present experimental condition. ${ }^{182}$ Although, this catalytic system exhibited high conversion towards the oxidation of ethylbenzene, the use of $\mathrm{Cd}$ may be a drawback from a green perspective.

Porous metalloporphyrinic frameworks have been constructed between metal 5,10,15,20-tetrakis(3,5-biscarboxylphenyl)porphyrin (metal : $\mathrm{Mn}^{2+}$ or $\mathrm{Cd}^{2+}$ ) and $\mathrm{MnCl}_{2}$ or $\mathrm{CdCl}_{2}$ and their activity tested in the oxidation of alkylbenzenes. ${ }^{183}$ The self-assembled porous metalloporphyrinic frameworks $\left[\mathrm{Mn}_{5} \mathrm{Cl}_{2}(\mathrm{MnCl}-\mathrm{OCPP})-\right.$ $\left.(\mathrm{DMF})_{4}\left(\mathrm{H}_{2} \mathrm{O}\right)_{4}\right] \cdot 2 \mathrm{DMF} \cdot 8 \mathrm{CH}_{3} \mathrm{COOH} \cdot 14 \mathrm{H}_{2} \mathrm{O}\left(\mathrm{ZJU}-18 ; \mathrm{M}=\mathrm{Mn}{ }^{\mathrm{III}} \mathrm{Cl} ; \mathrm{ZJU}=\right.$ Zhejiang University $)$, $\left[\mathrm{Mn}_{5} \mathrm{Cl}_{2}(\mathrm{Ni}-\mathrm{OCPP})\left(\mathrm{H}_{2} \mathrm{O}\right)_{8}\right] \cdot 7 \mathrm{DMF}-\cdot 6 \mathrm{CH}_{3} \mathrm{COOH} \cdot 11 \mathrm{H}_{2} \mathrm{O}\left(\mathrm{ZJU}-19: \mathrm{M}=\mathrm{Ni}{ }^{\mathrm{II}}\right)$, and $\left[\mathrm{Cd}_{5} \mathrm{Cl}_{2}(\mathrm{MnCl}-\right.$ OCPP)-( $\left.\left.\mathrm{H}_{2} \mathrm{O}\right)_{6}\right] \cdot 13 \mathrm{DMF} \cdot 2 \mathrm{CH}_{3} \mathrm{COOH} \cdot 9 \mathrm{H}_{2} \mathrm{O}\left(\mathrm{ZJU}-20 ; \mathrm{M}=\mathrm{Mn}{ }^{\mathrm{III}} \mathrm{Cl}\right)$ (OCPP: metalloporphyrin octacarboxylates) are isostructural as revealed by their single X-ray crystal structures. ${ }^{183}$ The catalytic activity of these MOFs was determined in a mixed solvent of acetonitrile, acetic acid and water at $65{ }^{\circ} \mathrm{C}$ for $18 \mathrm{~h}$ using TBHP as oxidant. ZJU-18 efficiently catalyzed the oxidation of ethylbenzene to acetophenone quantitatively with $100 \%$ selectivity while ZJU-19 having $\mathrm{Ni}^{\mathrm{II}}$-OCPP porphyrin centers displayed negligible catalytic activity and only $9 \%$ acetophenone was formed. The significant activity difference between ZJU-18 and ZJU-19 clearly indicates the active participation of the $\mathrm{Mn}{ }^{\mathrm{III}} \mathrm{Cl}-\mathrm{OCPP}$ sites within $\mathrm{ZJU}-18$ as the efficient catalytic sites. It is also possible in view of the activity of other MOFs that do not contain porphyrin previously commented that the manganese sites on the $\mathrm{SBU}$ nodes $\mathrm{Mn}_{3}^{\mathrm{II}}(\mathrm{COO})_{4} \mathrm{Cl}_{2}$ and $\mathrm{Mn}_{3}{ }_{3}(\mathrm{COO})_{4}\left(\mu-\mathrm{H}_{2} \mathrm{O}\right)_{2}\left(\mathrm{H}_{2} \mathrm{O}\right)_{6}$ within $\mathrm{ZJU}-18$ might also play a role on the oxidation. On the other hand, it was observed that ZJU-20 showed $69 \%$ conversion. Independent experiments with $\mathrm{MnCl}_{2}, \mathrm{Mn}-\mathrm{Me}_{8} \mathrm{OCPP}$, or $\mathrm{Ni}-\mathrm{Me}_{8} \mathrm{OCPP}$ resulting in 5, 16 and $1 \%$ conversion of ethylbenzene, respectively, indicate that $\mathrm{Mn}^{\mathrm{III}} \mathrm{Cl}-\mathrm{OCPP}$ sites within ZJU-18 play the most important role in achieving a highly efficient and selective oxidation of ethylbenzene. Using ZJU-18 as catalyst, propylbenzene, tetralin and fluorene were oxidized to their corresponding 
ketones with 74, 58 and $42 \%$ conversion. ${ }^{183}$ ZJU-18 exhibited 18 and $16 \%$ conversion for larger substrates like diphenylmethane and 4-ethylbiphenyl, respectively, while the molecular $\mathrm{MnCl}-\mathrm{Me}_{8} \mathrm{OCPP}$ catalyst showed 26 and $28 \%$ conversion, respectively. These experiments clearly demonstrated the diffusion limitation encountered by larger molecules and that in this case the catalytic reaction should take place mainly on the external surface of the crystallites. The adsorption/esorption experiments clearly showed that ZJU-18 preferably takes up 23 molecules of ethylbenzene over 5 molecules of acetophenone. ${ }^{183}$ In contrast, 4-ethylbiphenyl is not adsorbed inside the pores of ZJU-18. The catalyst (ZJU-18) was reused for fifteen times without any noticeable decrease in conversion, while the recovered homogeneous $\mathrm{MnCl}-$ $\mathrm{Me}_{8} \mathrm{OCPP}$ lost its activity after three cycles indicating the superior performance of porous MOF structures, although it may suffer from diffusion limitations for substrates of large dimensions. ${ }^{183}$ The TON observed for the oxidation of ethylbenzene using ZJU-18 as catalyst was found to be 8076 . Powder XRD showed that the structural integrity was maintained during the catalytic cycle, without detecting leaching of metal.

MIL-101(Cr) supported Au-Pd catalysts were prepared by using a simple colloidal deposition method with poly(vinyl alcohol) as protecting agent and its catalytic activity was tested in the one-pot synthesis of aromatic esters through C-H activation. ${ }^{184}$ Although the mechanism of incorporation of Au-Pd nanoparticles from the polymer stabilized colloid inside the pores of MOF is still to be determined, the average size of the resulting Au-Pd nanoparticles was around $3 \mathrm{~nm}$. Toluene and methanol were selected as substrates for the synthesis of methyl benzoate in the presence of molecular oxygen as oxidant (Scheme 36). Methyl benzoate was obtained as the main oxidation product, accompanied by other byproducts such as benzaldehyde, benzaldehyde dimethyl acetal, and benzyl benzoate. Au/MIL-101(Cr) exhibited $1.4 \%$ conversion of toluene with $17 \%$ selectivity toward methyl benzoate. A moderate conversion (34\%) of toluene was observed with Au-Pd 1:1.5 molar ratio in $\mathrm{Au}-$ 
Pd/MIL-101(Cr) with $88 \%$ selectivity to methyl benzoate. A further increase in Pd content resulted in reduced conversion (33.8\%) and selectivity (71.9) to methyl benzoate. An increase in $\mathrm{O}_{2}$ pressure resulted in higher conversion $(50.5 \%)$, but remarkably reduced selectivity $(71.5$ \%) to methyl benzoate. However, increasing the temperature to $120{ }^{\circ} \mathrm{C}$ improved toluene conversion to $50.3 \%$, while maintaining high selectivity $(84.2 \%)$ to methyl benzoate. A maximum of $96.9 \%$ conversion of toluene was observed with $96.6 \%$ selectivity to methyl benzoate at Au-Pd molar ratio 1:1.5 and $120^{\circ} \mathrm{C}$. The use of alloy nanoparticles is a field to be developed in order to increase the efficiency and selective of pure metal nanoparticles and this study exemplifies how the composition of the metal alloy can be tuned to optimize the catalyst performance in oxidation reactions.

The electronic factors of the substituents influence the reactivity of the aromatic substrate that increases as the electron donor ability of the substituent. Thus, electron-deficient aromatic hydrocarbon such as 4 -fluorotoluene showed $75 \%$ conversion with $88 \%$ selectivity to the methyl ester, while 4-methoxytoluene exhibited $93.9 \%$ conversion with $98 \%$ selectivity. The reaction can also be performed using other alcohols besides methanol. Under the optimized reaction conditions, the reaction between toluene and various aliphatic alcohols including methanol, ethanol, n-propanol, and isopropyl alcohol smoothly underwent esterification with 96.9, 99, 94.5 and $92.8 \%$ conversions and 96.6, 95.6, 89.3, and 84.6\% selectivities, respectively, towards the desired esters. On the other hand, the conversion was reduced for the reaction between toluene and n-butanol to $80.2 \%$ with $80 \%$ selectivity suggesting the steric hindrance of the alcohol.

MIL-101(Cr) containing Au-Pd nanoparticles exhibited stability as catalyst and coudl be reused in three consecutive batch reactions for the synthesis of methyl benzoate. The crystalline structure of the catalyst also remained mostly unchanged after three catalytic cycles. No metal was detected in the filtered solution indicating the heterogeneity of the process. TEM 
images of the reused catalysts did not reveal any appreciable changes in nanoparticle size in fresh and reused catalyst. These results confirm the stability of the catalyst under the experimental conditions.

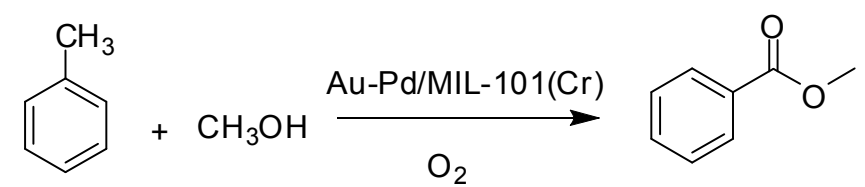

Scheme 36. Oxidative esterification of toluene with methanol catalyzed by Au-Pd/MIL-101(Cr).

\section{Oxidation of alcohols}

A ruthenium trichloride complex has been loaded into MOF-253 by post-synthetic modification to give MOF-253-Ru through the coordination of ruthenium complex with open bipyridine sites (Scheme 37). ${ }^{185}$ Two samples were prepared with 7 and $13 \mathrm{~mol} \%$ of Ru content (i.e., Ru complexes occupied 7 and 13\% of the bpydc linkers in the MOF, respectively) and named as MOF-253-Ru7 and MOF-253-Ru13.

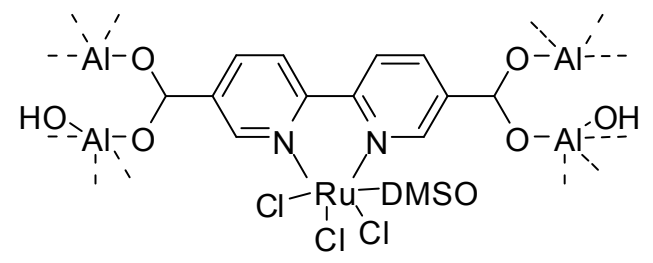

Scheme 37. Post synthetic modification of MOF-253 by Ru complex coordinated to the bpy site.

Oxidation of 1-phenylethanol was selected as model reaction using diacetoxyiodobenzene as oxidant, achieving quantitative conversion at room temperature with $0.5 \mathrm{~mol} \% \mathrm{MOF}-253-\mathrm{Ru} 7 .{ }^{185}$ Benzylic secondary alcohols containing aromatic rings bearing electron-donating and electron-accepting groups were also oxidized to the corresponding ketones with nearly full conversion with $0.5 \mathrm{~mol} \% \mathrm{MOF}-253-\mathrm{Ru} 7{ }^{185}$ A variety of aliphatic cyclic and acyclic sec-alcohols were also oxidized in excellent yield under identical conditions. 
Moreover, the catalyst exhibits chemoselectivity for alcohol oxidation in several allylic alcohols, affording the corresponding unsaturated ketone with moderate to good yields by using $1 \mathrm{~mol} \%$ MOF-253-Ru7. ${ }^{185}$ Benzyl alcohol and its derivatives were oxidized to their corresponding aldehydes in high conversion and selectivities at room temperature by using 1 mol \% MOF-253-Ru7. ${ }^{185}$ On the other hand, oxidation of primary aliphatic alcohols, such as 1nonanol or 2,2-dimethylpropan-1-ol afforded only traces of the corresponding aldehydes and thus further development is still necessary to make the systeme general for any alcohol oxidation. Interestingly, a more complex alcohol, cholestanol, was oxidized to the corresponding ketone (Scheme 38) in excellent yield in the presence of $0.5 \mathrm{~mol} \% \mathrm{Ru}$ using MOF-253-Ru7 as catalyst. ${ }^{185}$ ICP-OES analysis revealed the presence of 0.9 and $1.6 \mathrm{ppm}$ of $\mathrm{Al}$ and $\mathrm{Ru}$, respectively, in the supernatant solution which corresponds to 0.1 and $0.7 \%$ of the initial amounts of $\mathrm{Al}$ and $\mathrm{Ru}$, respectively, in MOF-253-Ru7. ${ }^{185}$ The catalyst was reused six times with a moderate decrease in conversion from 97 to $85 \%$ between the first and the sixth run, respectively. ${ }^{185}$ Analysis of the used catalyst revealed no change in either crystallinity or surface area. On the other hand, the Ru content decreased from 7 to $5 \mathrm{~mol} \%$ after six cycles, according to elemental analysis. Although the present catalytic system showed reasonably high activity for various primary, secondary and cyclic alcohols, the use of dichloromethane as solvent and a bulky oxidant generating large amouns of waste are the serious drawbacks of this protocol. In this regard, it would be of interest to develop a process in which $\mathrm{PhI}(\mathrm{OAc})_{2}$ is used in catalytic amounts and molecular oxygen is the terminal oxidizing reagent being consumed.
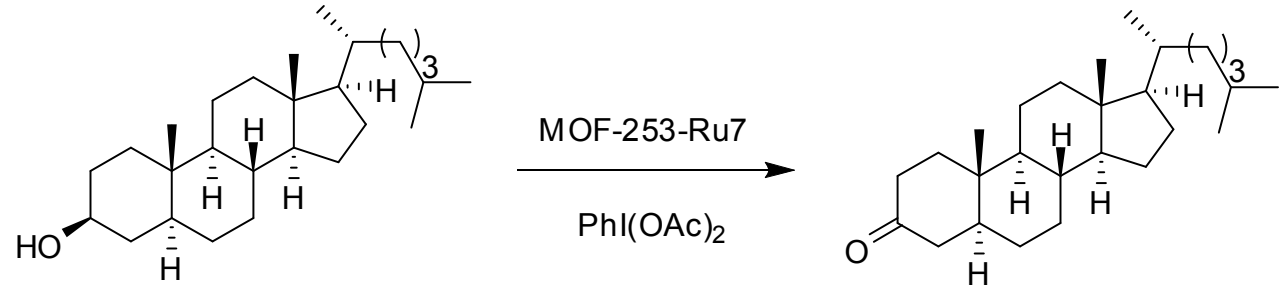

Scheme 38. Oxidation of cholestanol to cholestanone catalyzed by MOF-253-Ru7. 
As it has been already discussed in the oxidation of styrene to benzaldehyde catalyzed by $\mathrm{Fe} / \mathrm{MIL}-101(\mathrm{Cr})$, this catalyst was also found to catalyze oxidation of benzyl alcohols to their corresponding benzaldehydes using hydrogen peroxide in water under microwave irradiation at $110-120{ }^{\circ} \mathrm{C} .{ }^{178}$ Benzyl alcohols with electron donating and electron withdrawing groups were smoothly oxidized to their corresponding benzaldehydes in quantitative yields. ${ }^{178}$ Methyl substitution on the benzyl alcohol was tolerated during the oxidation reaction. On the other hand, cyclohexanol was oxidized exclusively with $77 \%$ conversion. ${ }^{178} 1$-Hexanol and 6-methyl-1-heptanol were oxidized with less than $20 \%$ conversion and around $65 \%$ selectivity to their aldehydes, respectively. ${ }^{178}$ Cycloheptanol showed around $10 \%$ conversion but cycloheptanone was not observed. ${ }^{178}$ The failure to oxidize aliphatic alochols leaves clearly a gap in the scope of the process for further development of a highly efficient catalytic system for the oxidation of linear alcohols to their corresponding aldehydes. One advantage of $\mathrm{Fe} / \mathrm{MIL}-101(\mathrm{Cr})$ as catalyst is that it does not require base as promoter. The catalyst was stable and can be reused for three cycles without any significant change in its activity and without detecting iron in the supernantant as evidenced by ICP-MS, thus, ruling out the possibility of homogeneous catalysis by dissolved iron species. ${ }^{178}$ Powder XRD and nitrogen adsorption measurements showed that the structural integrity was maintained during the catalytic studies. ${ }^{178}$

\section{Synthesis of fine chemicals through reduction reactions}

This section describes the use of MOFs as heterogeneous catalysts for the synthesis of fine chemicals involving reduction reactions (Table 3). They include hydrogenation of $\mathrm{C}=\mathrm{C}$ double bonds, reduction of alkyl aryl ketone, cyclohexanone and dehalogenation of aryl halides. The emphasis has been made on addressing the occurrence of metal leaching as most 
of the fine chemicals are supposed to be synthesized within the acceptable limit of metal impurities. In addition, catalyst stability is also discussed.

Table 3. List of MOFs as solid catalysts for the synthesis of fine chemicals through reduction reactions.

\begin{tabular}{|l|l|l|l|}
\hline MOF formula & Reaction & Reductant & Ref. \\
\hline $\mathrm{Pd} / \mathrm{MOF}-5$ & hydrogenation of ethyl cinnamate & $\mathrm{H}_{2}$ & 192 \\
\hline$\left[\mathrm{ZnLCl}_{2}\right] \cdot 8 \mathrm{H}_{2} \mathrm{O}^{\mathrm{a}}$ & $\begin{array}{l}\text { hydrogenation of ethyl cinnamate } \\
\text { and nitrobenzene }\end{array}$ & $\mathrm{H}_{2}$ & 193 \\
\hline $\begin{array}{l}{\left[\mathrm{Ca}(\text { hfipbb })\left(\mathrm{H}_{2} \mathrm{hfipbb}\right)_{0.5}\right.} \\
\left.\left(\mathrm{H}_{2} \mathrm{O}\right)\right] \cdot 0.7 \mathrm{C}_{3} \mathrm{H}_{6} \mathrm{O}\end{array}$ & hydrogenation of styrene & $\mathrm{H}_{2}$ & 194 \\
\hline $\mathrm{Pd}_{3} \mathrm{Ni}{ }_{2} @$ MIL-101 & hydrogenation of cyclohexanone & $\mathrm{H}_{2}$ & 195 \\
\hline $\mathrm{SIM}-1 / \gamma-$ alumina & hydrogenation of acetophenone & $\left(\mathrm{CH}_{3}\right)_{2} \mathrm{CHOH} / \mathrm{KOH}$ & 196 \\
\hline $\mathrm{Pd} / \mathrm{NH} \mathrm{H}_{2}-\mathrm{MIL}-101(\mathrm{Cr})$ & dehalogenation of arylhalide & ammonium formate & 197 \\
\hline
\end{tabular}

${ }^{\mathrm{a}} \mathrm{L}$ : 1,1'-methylenebis-(3-(4-carboxy-2-methylphenyl)-1H-imidazol-3-ium).

\section{Hydrogenation of $C=C$ bond}

Coprecipitation of zinc nitrate hexahydrate, 1,4-benzenedicarboxylic acid and palladium(II) nitrate dihydrate as precursors has been used to prepare a $0.5 \mathrm{wt} \%$ palladium embedded on MOF-5. ${ }^{192}$ The activity of palladium embedded on MOF-5 for the hydrogenation of ethyl cinnamate by hydrogen was twice as high as that of a commercial $\mathrm{Pd} / \mathrm{C}$ catalyst with the same Pd content. ${ }^{192}$ No leaching of palladium was observed during hydrogenation. ${ }^{192}$ Palladium embedded on MOF-5 did not show any loss in activity in repeated runs. ${ }^{192}$ However a complete loss of micropore volume was observed, suggesting the complete collapse of the crystal structure. Comparison of these adsorption data with the catalytic activity would indicate that the active palladium is not located inside the micropores, but probably deposited on the outer surface of the crystals. Additional characterization data are, however, needed to explain the catalytic activity in the absence of porosity. 
A metal-organic nanotube (MONT) with the formula $\left[\mathrm{ZnLCl}_{2}\right] \cdot 8 \mathrm{H}_{2} \mathrm{O}$ was synthesized by linking up the bent organic ligand 1,1'-methylenebis-(3-(4-carboxy-2-methylphenyl)-1Himidazol-3-ium) with tetra-coordinated zinc cations. ${ }^{193}$ It was shown that the imidazolium moieties in the $1 \mathrm{D}$ channels of $\left[\mathrm{ZnLCl}_{2}\right] \cdot 8 \mathrm{H}_{2} \mathrm{O}$ are readily available to be deprotonated for the formation of $\mathrm{N}$-heterocyclic carbenes by postmodification by treating $\left[\mathrm{ZnLCl}_{2}\right] \cdot 8 \mathrm{H}_{2} \mathrm{O}$ with $\mathrm{Pd}(\mathrm{OAc})_{2}$ in THF. ${ }^{193}$ The solid $\left[\mathrm{ZnLCl}_{2}\right] \cdot 8 \mathrm{H}_{2} \mathrm{O}-\mathrm{Pd}$ is more stable than compound $\left[\mathrm{ZnLCl}_{2}\right] \cdot 8 \mathrm{H}_{2} \mathrm{O} .{ }^{193}$ Hence, the catalytic activity of $\left[\mathrm{ZnLCl}_{2}\right] \cdot 8 \mathrm{H}_{2} \mathrm{O}-\mathrm{Pd}$ was tested in the hydrogenation of $\mathrm{C}=\mathrm{C}$ double bond and nitrobenzene with molecular hydrogen as reducing agent at room temperature. ${ }^{193}$ (E)-ethyl cinnamate and its derivatives, styrene and its derivatives, cycloalkenes and linear alkenes were successfully hydrogenated to their corresponding alkanes in quantitative yields with high selectivity at room temperature. ${ }^{193}$ For example, for the hydrogenation of (E)-ethyl cinnamate the TON reaches values of 13746 without loss of the catalytic efficiency suggesting its high stability. ${ }^{193}$ The solid $\left[\mathrm{ZnLCl}_{2}\right] \cdot 8 \mathrm{H}_{2} \mathrm{O}-\mathrm{Pd}$ was reused six times without any decrease in its catalytic activity. The catalytic potential of $\left[\mathrm{ZnLCl}_{2}\right] \cdot 8 \mathrm{H}_{2} \mathrm{O}-\mathrm{Pd}$ was further demonstrated by reduction of nitrobenzene with hydrogen under atmospheric pressure at room temperature resulting in quantitative yield of aniline. ${ }^{193}$

Solvothermal reaction of $\mathrm{Ca}(\mathrm{OAc})_{2} \cdot \mathrm{H}_{2} \mathrm{O}$ and 4,4'-(hexafluoroisopropylidene) bis(benzoic acid) in a water/acetone mixture resulted in the formation of a new MOF with the formula $\left[\mathrm{Ca}\right.$ (hfipbb) $\left.\left(\mathrm{H}_{2} \text { hfipbb }\right)_{0.5}\left(\mathrm{H}_{2} \mathrm{O}\right)\right] \cdot 0.7 \mathrm{C}_{3} \mathrm{H}_{6} \mathrm{O} .{ }^{194}$ This catalyst was tested for the hydrogenation of styrene to ethylbenzene using hydrogen as reducing agent in toluene medium (Scheme 39). Using this catalyst, styrene showed $100 \%$ conversion with complete selectivity towards ethylbenzene at $100{ }^{\circ} \mathrm{C}$ with TOF being $254 \mathrm{~h}^{-1}$, while in contrast calcium acetate showed only $20 \%$ conversion. On the other hand, $\alpha$-methylstyrene offered $60 \%$ conversion towards isopropylbenzene with $100 \%$ selectivity. Although the catalyst showed no leaching of 
active sites, the catalytic activity decreased from 100 to $70 \%$ from first to second run and remained steady for the third and the fourth runs. Powder XRD, DRS and FT-IR showed no evidence for the structural damage or the presence of any byproducts.

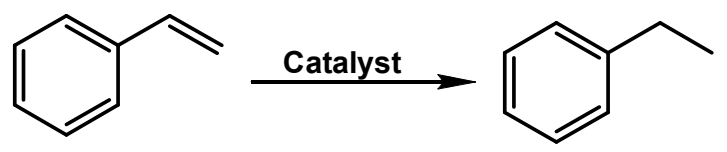

Scheme 39. Reduction of styrene to ethylbenzene catalyzed by $\left[\mathrm{Ca}\left(\right.\right.$ hfipbb) $\left.\left(\mathrm{H}_{2} \text { hfipbb }\right)_{0.5}\left(\mathrm{H}_{2} \mathrm{O}\right)\right] \cdot 0.7 \mathrm{C}_{3} \mathrm{H}_{6} \mathrm{O}$

\section{Hydrogenation of ketones}

The generation of Ni/Pd NPs of different compositions and sizes limited by the cavity dimensions embedded within MIL-101 and their synergism in the catalytic hydrogenation of cyclohexanone (Scheme 40) and 3-heptanone has been reported. ${ }^{195}$ Neither 3-heptanone nor cyclohexanone are reduced by the Ni@MIL-101 catalyst under the conditions used. ${ }^{195}$ Also, the activity of Pd@MIL-101 was lower than that of Ni/Pd@MIL-101, although comparable to that of $\mathrm{Pd}$ on activated carbon $(\mathrm{Pd} / \mathrm{C}) .{ }^{195} \mathrm{Ni}_{2} \mathrm{Pd}_{3} @$ MIL-101 catalyst showed high activity for cyclohexanone reduction with 100 \% conversion, whereas $\mathrm{Ni}_{3} \mathrm{Pd}_{2} @ \mathrm{MIL}-101$ resulted in around $76 \%$ conversion of cycloheptanone. ${ }^{195}$ Under analogous conditions, lower conversions of 3heptanone was observed by the mixture of pure Pd@MIL-101 and Ni@MIL-101 catalysts in comparison to that of the bimetallic cavity-conformed $\mathrm{Ni}_{2} \mathrm{Pd}_{3} @ \mathrm{MIL}-101$ catalyst indicating a synergistic effect due to the formation of nanoalloys. ${ }^{195} \mathrm{Ni}_{2} \mathrm{Pd}_{3} @$ MIL-101 resulted in $80 \%$ conversion of 3-heptanone at $35{ }^{\circ} \mathrm{C} .{ }^{195}$ Reusability tests indicated no drop in conversion of 3heptanone up to 10 cycles, proving the robust nature of this catalyst. ${ }^{195}$ Powder XRD showed no change in the structural integrity of MIL-101 and TEM analysis show no increase in the metal particle size after many cycles. ${ }^{195}$

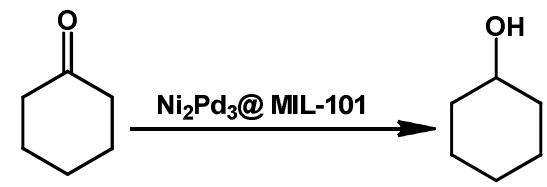


Scheme 40. Hydrogenation of cyclohexanone to cyclohexanol catalyzed by $\mathrm{Ni}_{2} \mathrm{Pd}_{3} @ \mathrm{MIL}-101$.

Substituted imidazolate material (SIM-1: $\left.\mathrm{Zn}\left(\mathrm{C}_{5} \mathrm{H}_{5} \mathrm{~N}_{2} \mathrm{O}\right)_{2}\right]$ ) belongs to the class of ZIFs and is isostructural to ZIF-8. It consists of $\mathrm{Zn}_{4}$ tetrahedra linked by carboxylimidazolate. SIM-1 was supported on $\gamma$-alumina beads and this was used as catalyst for the reduction of acetophenone to 1-phenylethanol by hydrogen transfer from isopropyl alcohol using potassium hydroxide as base (Scheme 41). ${ }^{196}$ Using 5 mol \% of SIM-1 supported on $\gamma$-alumina in the presence of $25 \mathrm{~mol} \%$ of potassium hydroxide at $80{ }^{\circ} \mathrm{C}$, more than $90 \%$ of the substrate is converted, whereas less than $10 \%$ conversion is observed when the reaction is performed with alumina beads alone. ${ }^{196}$ Under the same conditions, the SIM-1 exhibited similar, but lower, catalytic activity to the SIM-1/alumina composite with $87 \%$ conversion, indicating the superior performance of SIM-1/alumina composite. ${ }^{196}$ The catalyst can be easily separated from the reaction mixture and can be recycled. ${ }^{196}$

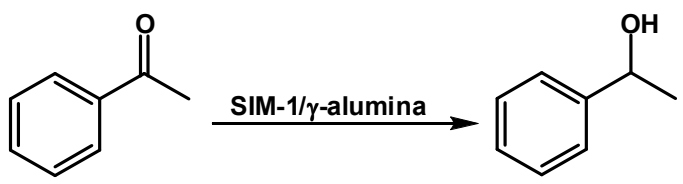

Scheme 41. Reduction of acetophenone catalyzed by SIM-1/ $\gamma$-alumina.

\section{Dehalogenation of arylhalides}

Highly dispersed palladium nanoparticles have been encapsulated in the mesoporous cages of amine functionalized MOFs namely $\mathrm{NH}_{2}-\mathrm{MIL}-101(\mathrm{Cr}) .{ }^{197}$ The particle size $(2.49 \mathrm{~nm})$ of the obtained Pd NPs was in good agreement with the cage diameters of MOFs (2.9 nm and $3.4 \mathrm{~nm}) . \mathrm{Pd} / \mathrm{NH}_{2}-\mathrm{MIL}-101(\mathrm{Cr})$ was tested as catalyst for the dehalogenation of aryl halides in the presence of ammonium formate using water as medium at room temperature (Scheme 42). ${ }^{197}$ Quantitative yields were achieved for many aryl chlorides and its derivatives. ${ }^{197}$ Under the optimized conditions, 4-chlorophenol resulted in $98 \%$ of phenol. ${ }^{197}$ Leaching tests showed no evidence for the presence of palladium and chromium and ICP-AES analysis showed 0.5 ppm of Pd which is within the acceptable limit of metal impurities in the synthesis of fine 
chemicals. ${ }^{197}$ Further, it was demonstrated that this leached Pd does not contribute to the formation of the product. The catalyst can be reused five times with a slight decrease of yield. ${ }^{197}$ In addition, powder XRD showed that the crystalline structure is mostly retained after five cycles.

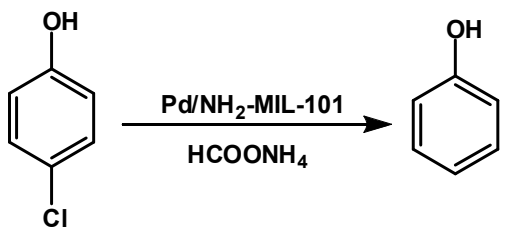

Scheme 42. Dehalogenation of 4-chlorophenol catalyzed by $\mathrm{Pd} / \mathrm{NH}_{2}-\mathrm{MIL}-101(\mathrm{Cr})$.

\section{Final Conclusions and Future Prospects}

In the above sections, we have provided examples of MOFs as solid catalysts in liquid phase reactions for the production of fine chemicals. When the catalytic performance of MOF has been compared with that of zeolites or even with that of homogeneous soluble salts, it has been frequently observed that MOFs exhibit higher activity. This enhanced performance has been explained in terms of accessibility of active sites with respect to that of zeolites and arising from the lesser tendency of MOFs to undergo deactivation compared with analogous homogeneous salts easily undergoing aggregation during operation of the catalytic steps. Very frequently homogeneous catalysts undergo deactivation by aggregation and decomposition under the reaction conditions. In these cases, immobilization in a porous framework can be a viable strategy to increase the stability of the active sites, although some diffusion restrictions could apply to large substrates, depending on the pore size. Thus, very commonly, MOFs are the best choice as catalysts considering activity and stability.

With respect to the access of the internal sites or the exclusive operation of the catalytic centers located on the external surface of the MOF crystallites, there are contrasting examples supporting internal diffusion or the lack of activity of internal sites. It is very likely that the reaction conditions (mainly temperature and solvents) and the dimensions of the substrates and 
reagents with respect to the size of the pores are the crucial parameters determining the accessibility of the internal sites. Therefore, the issue of the catalytic activity of the internal sites should be addressed for each reaction under less favorable diffusion conditions and MOFs with pore dimensions that suit with reactants and products should be the preferred options.

There are sufficient reports of the use of chiral MOFs as enantioselective solid catalysts to consider that this is a viable strategy that could be successfully implemented in many more processes. In addition, the mild reaction conditions required for asymmetric induction are specially suited to guarantee the framework stability of MOFs. Thus, it can be easily anticipated that this asymmetric catalysis using MOFs is an area that will grow considerably in the next years, particularly considering that in aluminosilicates and other metal oxides the unavoidable presence of adventitious acid or basic sites is highly detrimental for asymmetric induction. For this reason, preparation of enantioselective catalysts based on these metal oxides has always some step aimed at passivating these undesirable sites, a step that may be unnecessary for MOFs.

Although much work in this area is still desirable, MOFs also offer considerable promise as catalysts for tandem reactions. A few reports, such as in the deacetalization/Knoevenagel reactions and the Meinwald epoxide rearrangement/Knoevenagel condensation, have already shown the potential of MOFs. These examples support the feasibility of using MOF for developing tandem processes, but it is clear that this field remains almost totally unexplored and will be of large industrial impact. Considering the interest of tandem processes in catalysis and that MOFs are currently attracting much attention, it appears logical to expect many more research effort on the design and use of MOFs as catalysts for more challenging tandem reactions, particularly for individual steps requiring high selectivity, with reactions of industrial relevance. 
Finally, one crucial point that is poorly addressed or ignored in most of the reports is MOF stability and deactivation processes. All the catalysts eventually deactivate and the point is to assess the maximum productivity of a catalyst before deactivation and in which extent the material can be reactivated. Due to the nature of the coordination metal ligand bonds, framework stability of MOFs is considerably lower than silicates and these materials have to be always extensively surveyed for stability. We notice, however, that this lower thermal and chemical stability of MOFs does not necessarily imply that they are useless catalysts, but only that the reaction conditions have to be compatible with a full stability of the structure considering limits in the reaction conditions. Low reaction temperatures using non-coordinating solvents and adequate reagents are favorable for MOF stability. In this sense, it should be commented that reusability tests do not provide sufficient information about the catalyst stability. Time-conversion plots, in which initial reaction rates of the fresh and used material can be compared are far more informative than providing conversions at final times. Maximum productivity in terms of maximum mass of product per mass unit of MOF catalyst can be the figure of merit to compare with other catalyst, together with availability and price. Continuous flow studies are also best suited to determine catalyst stability but they have not been extensively used to address the decay in the catalytic activity of MOFs.

A last remark would be that since the added value of some fine chemicals is sufficiently high, they offer a real possibility for using as catalysts materials such as MOFs. One starting point could be those industrial processes that are already using a transition metal salt as homogeneous catalyst and try to design a MOF with active sites mimicking those present in solution, but enjoying stability, recyclability and higher productivity. The key point for industrial application would be an improved performance of MOFs as catalysts with regard to other possible alternatives that should derive from catalyst stability and productivity. In view of 
this potential, it can be anticipated that MOFs will be increasingly used for the production of

fine chemicals and that the possibility to use them in a commercial process seems feasible.

\section{Acknowledgements}

A.D.M. thanks University Grants Commission (UGC), New Delhi for the award of Assistant Professorship under its Faculty Recharge Programme. Financial support by the Spanish DGI (CTQ-2012-32315 and Severo Ochoa) is gratefully acknowledged. The research leading to these results has received funding from the European Community's Seventh Framework Programme (FP7/2007-2013) under grant agreement no. 228862. J.Č. thanks the Czech Grant Agency for financial support (Centre of Excellence - P106/12/G015).

\section{References.}

1. A. Corma, Chem. Rev., 1995, 95, 559-614.

2. A. Corma, Chem. Rev., 1997, 97, 2373-2420.

3. I. Arends and R. A. Sheldon, Applied Catalysis a-General, 2001, 212, 175-187.

4. A. Corma, Journal of Catalysis, 2003, 216, 298-312.

5. J. Cejka, G. Centi, J. Perez-Pariente and W. J. Roth, Catalysis Today, 2012, 179, 2-15.

6. M. Guisnet and P. Magnoux, Applied Catalysis a-General, 2001, 212, 83-96.

7. M. J. Climent, A. Corma and S. Iborra, Chemical Reviews, 2011, 111, 1072-1133.

8. A. Corma, S. Iborra, S. Miquel and J. Primo, Journal of Catalysis, 1998, 173, 315-321.

9. R. A. Sheldon and R. S. Downing, Applied Catalysis a-General, 1999, 189, 163-183.

10. J. H. Clark, Accounts of Chemical Research, 2002, 35, 791-797.

11. R. A. Sheldon, Green Chemistry, 2007, 9, 1273-1283.

12. L. Ackermann, Chemical Reviews, 2011, 111, 1315-1345.

13. H. Clavier and H. Pellissier, Advanced Synthesis \& Catalysis, 2012, 354, 3347-3403.

14. F. Collet, C. Lescot and P. Dauban, Chemical Society Reviews, 2011, 40, 1926-1936.

15. S. F. Zhu and Q. L. Zhou, Accounts of Chemical Research, 2012, 45, 1365-1377.

16. T. Katsuki, Synlett, 2003, 281-297.

17. G. W. Coates and D. R. Moore, Angewandte Chemie-International Edition, 2004, 43, 6618-6639.

18. Y. Boutadla, D. L. Davies, S. A. Macgregor and A. I. Poblador-Bahamonde, Dalton Transactions, 2009, 5820-5831.

19. R. Kuwano, Synthesis-Stuttgart, 2009, 1049-1061.

20. R. Sen, S. Bhunia, D. Mal, S. Koner, Y. Miyashita and K. Okamoto, Langmuir, 2009, 25, 13667-13672).

21. X. B. Lu, W. M. Ren and G. P. Wu, Accounts of Chemical Research, 2012, 45, 17211735 .

22. F. Collet, R. H. Dodd and P. Dauban, Chemical Communications, 2009, 5061-5074. 
23. A. K. Cheetham, G. Ferey and T. Loiseau, Angewandte Chemie-International Edition, 1999, 38, 3268-3292.

24. G. Ferey, Chemical Society Reviews, 2008, 37, 191-214.

25. A. Dhakshinamoorthy, M. Alvaro and H. Garcia, Chem. Commun., 2012, 48, 11275.

26. A. Corma, H. Garcia and F. Xamena, Chemical Reviews, 2010, 110, 4606-4655.

27. M. Wang, M. H. Xie, C. D. Wu and Y. G. Wang, Chemical Communications, 2009, 2396-2398.

28. O. M. Yaghi, M. O'Keeffe, N. W. Ockwig, H. K. Chae, M. Eddaoudi and J. Kim, Nature, 2003, 423, 705-714.

29. S. Kitagawa, R. Kitaura and S. Noro, Angewandte Chemie-International Edition, 2004, 43, 2334-2375.

30. D. Farrusseng, S. Aguado and C. Pinel, Angewandte Chemie-International Edition, 2009, 48, 7502-7513.

31. H. Li, M. Eddaoudi, M. O'Keeffe and O. M. Yaghi, Nature, 1999, 402, 276-279.

32. M. Eddaoudi, D. B. Moler, H. L. Li, B. L. Chen, T. M. Reineke, M. O'Keeffe and O. M. Yaghi, Accounts of Chemical Research, 2001, 34, 319-330.

33. M. Eddaoudi, J. Kim, N. Rosi, D. Vodak, J. Wachter, M. O'Keeffe and O. M. Yaghi, Science, 2002, 295, 469-472.

34. S. T. Meek, J. A. Greathouse and M. D. Allendorf, Advanced Materials, 2011, 23, 249267.

35. J.-L. Wang, C. Wang and W. Lin, Acs Catalysis, 2012, 2, 2630-2640.

36. M. E. Davis and R. F. Lobo, Chemistry of Materials, 1992, 4, 756-768.

37. C. S. Cundy and P. A. Cox, Chemical Reviews, 2003, 103, 663-701.

38. M. E. Davis, Chemistry-a European Journal, 1997, 3, 1745-1750.

39. J. L. Paillaud, B. Harbuzaru, J. Patarin and N. Bats, Science, 2004, 304, 990-992.

40. C. Perego and R. Millini, Chemical Society Reviews, 2013, 42, 3956-3976.

41. W. J. Roth and J. Cejka, Catalysis Science \& Technology, 2011, 1, 43-53.

42. H. Furukawa and O. M. Yaghi, J. Am. Chem. Soc., 2009, 125, 8876-8883.

43. A. Dhakshinamoorthy, M. Alvaro, Y. K. Hwang, Y. K. Seo, A. Corma and H. Garcia, Dalton Transactions, 2011, 40, 10719-10724.

44. M. Ranocchiari, C. Lothschätz, D. Grolimund and J. A. van Bokhoven, Proceedings of the Royal Society A: Mathematical, Physical and Engineering Science, 2012, 468, 1985-1999.

45. A. Dhakshinamoorthy, M. Alvaro, A. Corma and H. Garcia, Dalton Transactions, 2011, 40, 6344-6360.

46. Y. Liu, W. M. Xuan and Y. Cui, Advanced Materials, 2011, 22, 4112-4135.

47. D. Zhao, D. J. Timmons, D. Q. Yuan and H. C. Zhou, Accounts of Chemical Research, 2010, 44, 123-133.

48. S. L. Qiu and G. S. Zhu, Coordination Chemistry Reviews, 2009, 253, 2891-2911.

49. P. I. Dalko and L. Moisan, Angewandte Chemie-International Edition, 2001, 40, 37263748.

50. R. Noyori and T. Ohkuma, Angewandte Chemie-International Edition, 2001, 40, 40-73.

51. R. Noyori, Angewandte Chemie-International Edition, 2002, 41, 2008-2022.

52. M. S. Taylor and E. N. Jacobsen, Angewandte Chemie-International Edition, 2006, 45, $1520-1543$.

53. J. Lee, O. K. Farha, J. Roberts, K. A. Scheidt, S. T. Nguyen and J. T. Hupp, Chemical Society Reviews, 2009, 38, 1450-1459.

54. C. Baleizao and H. Garcia, Chem. Rev., 2006, 106, 3987-4043.

55. T. Bein, Current Opinion in Solid State \& Materials Science, 1999, 4, 85-96.

56. C. E. Song and S. G. Lee, Chemical Reviews, 2002, 102, 3495-3524. 
57. D. Bradshaw, J. B. Claridge, E. J. Cussen, T. J. Prior and M. J. Rosseinsky, Accounts of Chemical Research, 2005, 38, 273-282.

58. G. Nickerl, A. Henschel, R. Grunker, K. Gedrich and S. Kaskel, Chemie Ingenieur Technik, 2011, 83, 90-103.

59. M. Yoon, R. Srirambalaji and K. Kim, Chemical Reviews, 2012, 112, 1196-1231.

60. L. F. Tietze, Chemical Reviews, 1996, 96, 115-136.

61. J. C. Wasilke, S. J. Obrey, R. T. Baker and G. C. Bazan, Chemical Reviews, 2005, 105, 1001-1020.

62. K. C. Nicolaou, D. J. Edmonds and P. G. Bulger, Angewandte Chemie-International Edition, 2006, 45, 7134-7186.

63. H. C. Guo and J. A. Ma, Angewandte Chemie-International Edition, 2006, 45, 354-366.

64. J. Christoffers, G. Koripelly, A. Rosiak and M. Rossle, Synthesis-Stuttgart, 2007, 12791300 .

65. M. Heitbaum, F. Glorius and I. Escher, Angewandte Chemie-International Edition, 2006, 45, 4732-4762.

66. A. Dhakshinamoorthy and H. Garcia, Chemical Society Reviews, 2012, 41, 5262-5284.

67. A. Dhakshinamoorthy, M. Opanasenko, J. Cejka and H. Garcia, Advanced Synthesis \& Catalysis, 2013, 355, 247-268.

68. J. Juan-Alcaniz, J. Gascon and F. Kapteijn, Journal of Materials Chemistry, 2012, 22, 10102-10118.

69. H. L. Ngo, A. G. Hu and W. B. Lin, Journal of Molecular Catalysis a-Chemical, 2004, 215, 177-186.

70. C. D. Wu, A. Hu, L. Zhang and W. B. Lin, Journal of the American Chemical Society, 2005, 127, 8940-8941.

71. C. D. Wu and W. B. Lin, Angewandte Chemie-International Edition, 2007, 46, 10751078.

72. L. Q. Ma, C. D. Wu, M. M. Wanderley and W. B. Lin, Angewandte ChemieInternational Edition, 2010, 49, 8244-8248.

73. L. Q. Ma, J. M. Falkowski, C. Abney and W. B. Lin, Nature Chemistry, 2010, 2, 838846.

74. S. K. Yoo, J. Y. Ryu, J. Y. Lee, C. Kim, S. J. Kim and Y. Kim, Dalton Transactions, 2003, 1454-1456.

75. S. J. Hong, J. Y. Ryu, J. Y. Lee, C. Kim, S. J. Kim and Y. Kim, Dalton Transactions, 2004, 2697-2701.

76. S. J. Hong, J. S. Seo, J. Y. Ryu, J. H. Lee, C. Kim, S. J. Kim, Y. Kim and A. J. Lough, Journal of Molecular Structure, 2005, 751, 22-28.

77. D. Jiang, T. Mallat, F. Krumeich and A. Baiker, Journal of Catalysis, 2008, 257, 390 395.

78. K. Tanaka and K. Otani, New Journal of Chemistry, 2010, 34, 2389-2391.

79. M. J. Ingleson, J. P. Barrio, J. B. Guilbaud, Y. Z. Khimyak and M. J. Rosseinsky, Chemical Communications, 2008, 2680-2682.

80. K. Tanaka, S. Oda and M. Shiro, Chemical Communications, 2008, 820-822.

81. L. X. Shi and C. D. Wu, Chemical Communications, 2011, 47, 2928-2930.

82. J. M. Gu, W. S. Kim and S. Huh, Dalton Transactions, 2011, 40, 10826-10829.

83. J. Juan-Alcaniz, E. V. Ramos-Fernandez, U. Lafont, J. Gascon and F. Kapteijn, Journal of Catalysis, 2010, 269, 229-241.

84. M. B. Lalonde, O. K. Farha, K. A. Scheidt and J. T. Hupp, Acs Catalysis, 2012, 2, $1550-1554$.

85. M. C. Bernini, F. Gandara, M. Iglesias, N. Snejko, E. Gutierrez-Puebla, E. V. Brusau, G. E. Narda and M. A. Monge, Chemistry-a European Journal, 2009, 15, 4896-4905. 
86. X. Tan, L. Li, J. Y. Zhang, X. R. Han, L. Jiang, F. W. Li and C. Y. Su, Chemistry of Materials, 2012, 24, 480-485.

87. T. Sawaki and Y. Aoyama, Journal of the American Chemical Society, 1999, 121, 4793-4798.

88. T. Sawaki, T. Dewa and Y. Aoyama, Journal of the American Chemical Society, 1998, 120, 8539-8540.

89. T. Dewa and Y. Aoyama, Journal of Molecular Catalysis a-Chemical, 2000, 152, 257260.

90. M. Hatano and K. Ishihara, Chemical Communications, 2012, 48, 4273-4283.

91. R. Srirambalaji, S. Hong, R. Natarajan, M. Yoon, R. Hota, Y. Kim, Y. H. Ko and K. Kim, Chemical Communications, 2012, 48, 11650-11652.

92. A. Dhakshinamoorthy, M. Alvaro, H. Chevreau, P. Horcajada, T. Devic, C. Serre and H. Garcia, Catalysis Science \& Technology, 2012, 2, 324-330.

93. M. Opanasenko, M. Shamzhy and J. Cejka, Chemcatchem, 2013, 5, 1024-1031.

94. L. Kurfirtova, Y. K. Seo, Y. K. Hwang, J. S. Chang and J. Cejka, Catalysis Today, 2012, 179, 85-90.

95. L. T. L. Nguyen, C. V. Nguyen, G. H. Dang, K. K. A. Le and N. T. S. Phan, Journal of Molecular Catalysis a-Chemical, 2011, 349, 28-35.

96. L. T. L. Nguyen, K. K. A. Le and N. T. S. Phan, Chinese Journal of Catalysis, 2012, 33, 688-696.

97. Y. M. Zhang, V. Degirmenci, C. Li and E. J. M. Hensen, Chemsuschem, 2011, 4, 59-64.

98. S. Horike, M. Dinca, K. Tamaki and J. R. Long, Journal of the American Chemical Society, 2008, 130, 5854-+.

99. D. B. Dang, P. Y. Wu, C. He, Z. Xie and C. Y. Duan, Journal of the American Chemical Society, 2010, 132, 14321-14323.

100. M. Banerjee, S. Das, M. Yoon, H. J. Choi, M. H. Hyun, S. M. Park, G. Seo and K. Kim, Journal of the American Chemical Society, 2009, 131, 7524-+.

101. D. J. Lun, G. I. N. Waterhouse and S. G. Telfer, Journal of the American Chemical Society, 2011, 133, 5806-5809.

102. K. K. Tanabe and S. M. Cohen, Angewandte Chemie-International Edition, 2009, 48, 7424-7427.

103. K. Gedrich, M. Heitbaum, A. Notzon, I. Senkovska, R. Frohlich, J. Getzschmann, U. Mueller, F. Glorius and S. Kaskel, Chemistry-a European Journal, 2011, 17, 20992106.

104. J. S. Seo, D. Whang, H. Lee, S. I. Jun, J. Oh, Y. J. Jeon and K. Kim, Nature, 2000, 404, 982-986.

105. C. Chizallet, S. Lazare, D. Bazer-Bachi, F. Bonnier, V. Lecocq, E. Soyer, A. A. Quoineaud and N. Bats, Journal of the American Chemical Society, 2010, 132, 12365 12377.

106. I. H. Hwang, J. M. Bae, W. S. Kim, Y. D. Jo, C. Kim, Y. Kim, S. J. Kim and S. Huh, Dalton Transactions, 2012, 41, 12759-12765.

107. H. C. Guo, X. W. Wang and K. L. Ding, Tetrahedron Letters, 2004, 45, 2009-2012.

108. X. S. Wang, X. W. Wang, H. C. Guo, Z. Wang and K. L. Ding, Chemistry-a European Journal, 2005, 11, 4078-4088.

109. K. S. Jeong, Y. B. Go, S. M. Shin, S. J. Lee, J. Kim, O. M. Yaghi and N. Jeong, Chemical Science, 2011, 2, 877-882.

110. Y. K. Hwang, D. Y. Hong, J. S. Chang, S. H. Jhung, Y. K. Seo, J. Kim, A. Vimont, M. Daturi, C. Serre and G. Ferey, Angewandte Chemie-International Edition, 2008, 47, 4144-4148. 
111. S. N. Kim, S. T. Yang, J. Kim, J. E. Park and W. S. Ahn, Crystengcomm, 2012, 14, $4142-4147$.

112. M. Hartmann and M. Fischer, Microporous and Mesoporous Materials, 2012, 164, 3843.

113. S. Aguado, J. Canivet, Y. Schuurman and D. Farrusseng, Journal of Catalysis, 2011, 284, 207-214.

114. F. Xamena, F. G. Cirujano and A. Corma, Microporous and Mesoporous Materials, 2012, 157, 112-117.

115. L. T. L. Nguyen, K. K. A. Le, H. X. Truong and N. T. S. Phan, Catalysis Science \& Technology, 2012, 2, 521-528.

116. J. Park, J. R. Li, Y. P. Chen, J. M. Yu, A. A. Yakovenko, Z. Y. U. Wang, L. B. Sun, P. B. Balbuena and H. C. Zhou, Chemical Communications, 2012, 48, 9995-9997.

117. J. Gascon, U. Aktay, M. D. Hernandez-Alonso, G. P. M. van Klink and F. Kapteijn, Journal of Catalysis, 2009, 261, 75-87.

118. S. Hasegawa, S. Horike, R. Matsuda, S. Furukawa, K. Mochizuki, Y. Kinoshita and S. Kitagawa, Journal of the American Chemical Society, 2007, 129, 2607-2614.

119. M. Opanasenko, A. Dhakshinamoorthy, M. Shamzhy, P. Nachtigall, M. Horacek, H. Garcia and J. Cejka, Catalysis Science \& Technology, 2013, 3, 500-507.

120. A. Dhakshinamoorthy, M. Alvaro and H. Garcia, Applied Catalysis a-General, 2010, 378, 19-25.

121. L. T. L. Nguyen, T. T. Nguyen, K. D. Nguyen and N. T. S. Phan, Applied Catalysis aGeneral, 2012, 425, 44-52.

122. M. Opanasenko, M. Shamzhy, M. Lamac and J. Cejka, Catalysis Today, 2013, 204, 94100.

123. L. Alaerts, E. Seguin, H. Poelman, F. Thibault-Starzyk, P. A. Jacobs and D. E. De Vos, Chemistry-a European Journal, 2006, 12, 7353-7363.

124. M. Opanasenko, A. Dhakshinamoorthy, J.-S. Chang, H. Garcia and J. Čejka, Chemsuschem, 2013, 6, 865-871.

125. T. W. G. Solomons and C. B. ryhle, Organic Chemistry, 10th Edition International Student Version edn., Wiley, Hoboken, US, 2011.

126. C. Bolm, G. Schlingloff and K. Harms, Chemische Berichte-Recueil, 1992, 125, 11911203.

127. D. Seebach, D. A. Plattner, A. K. Beck, Y. M. Wang, D. Hunziker and W. Petter, Helvetica Chimica Acta, 1992, 75, 2171-2209.

128. T. Punniyamurthy and L. Rout, Coordination Chemistry Reviews, 2008, 252, 134-154.

129. A. Dhakshinamoorthy, M. Alvaro and H. Garcia, Chemistry-a European Journal, 2010, 16, 8530-8536.

130. D. Menche, F. Arikan, J. Li and S. Rudolph, Organic Letters, 2007, 9, 267-270.

131. G. Benz, R. Henning and J. P. Stasch, Angewandte Chemie-International Edition in English, 1991, 30, 1702-1704.

132. N. Ono, The Nitro Group in Organic Synthesis, Wiley-VCH, New York, 2001.

133. E. H. Cordes and H. G. Bull, Chemical Reviews, 1974, 74, 581-603.

134. F. Gandara, M. E. Medina, N. Snejko, B. Gomez-Lor, M. Iglesias, E. Gutierrez-Puebla and M. A. Monge, Inorganic Chemistry, 2008, 47, 6791-6795.

135. B. Gomez-Lor, E. Gutierrez-Puebla, M. Iglesias, M. A. Monge, C. Ruiz-Valero and N. Snejko, Chemistry of Materials, 2005, 17, 2568-2573.

136. A. Dhakshinamoorthy, M. Alvaro and H. Garcia, Advanced Synthesis \& Catalysis, 2010, 352, 3022-3030.

137. L. Bromberg and T. A. Hatton, Acs Applied Materials \& Interfaces, 2011, 3, 47564764 . 
138. C. Kohlpaintner, M. Schulte, J. Falbe, P. Lappe and J. Weber, in Ullmann's Encyclopedia of Industrial Chemistry, Wiley-VCH, weinheim, Editon edn., 2008.

139. H. Siegel and M. Eggersdorfer, in Ullmann's Encyclopedia of Industrial Chemistry, Wiley-VCH, Weinheim, Editon edn., 2005.

140. M. Bejblova, D. Prochazkova and J. Cejka, Chemsuschem, 2009, 2, 486-499.

141. S. Hanneseian, Pergamon Press:, New York, Editon edn., 1983.

142. J. Otera, Esterification: Methods, Reactions and Applications, Wiley-VCH, Weimheim, 2003.

143. F. F. Fleming, L. H. Yao, P. C. Ravikumar, L. Funk and B. C. Shook, Journal of Medicinal Chemistry, 2010, 53, 7902-7917.

144. A. K. Ghose, V. N. Viswanadhan and J. J. Wendoloski, Journal of Combinatorial Chemistry, 1999, 1, 55-68.

145. K. S. Atwal, G. C. Rovnyak, S. D. Kimball, D. M. Floyd, S. Moreland, B. N. Swanson, J. Z. Gougoutas, J. Schwartz, K. M. Smillie and M. F. Malley, Journal of Medicinal Chemistry, 1990, 33, 2629-2635.

146. K. S. Atwal, G. C. Rovnyak, J. Schwartz, S. Moreland, A. Hedberg, J. Z. Gougoutas, M. F. Malley and D. M. Floyd, Journal of Medicinal Chemistry, 1990, 33, 1510-1515.

147. L. Bromberg, Y. Diao, H. M. Wu, S. A. Speakman and T. A. Hatton, Chemistry of Materials, 2012, 24, 1664-1675.

148. E. Perez-Mayoral and J. Cejka, Chemcatchem, 2011, 3, 157-159.

149. E. Pérez-Mayoral, z. Musilová, B. Gil, B. Marszalek, M. Položij, P. Nachtigall and J. Čejka, Dalton Transactions, 2012, 41, 4036-4044.

150. M. Polozij, E. Perez-Mayoral, J. Čejka, J. Hermann and P. Nachtigall, Catalysis Today 2013, 204, 101- 107.

151. N. B. Pathan, A. M. Rahatgaonkar and M. S. Chorghade, Catalysis Communications, 2011, 12, 1170-1174.

152. J. Gershenzon and N. Dudareva, Nature Chemical Biology, 2007, 3, 408-414.

153. C. Milone, A. Perri, A. Pistone, G. Neri and S. Galvagno, Applied Catalysis a-General, 2002, 233, 151-157.

154. A. Dhakshinamoorthy, M. Alvaro and H. Garcia, Catalysis Science \& Technology, 2011, 1, 856-867.

155. A. Dhakshinamoorthy, M. Alvaro and H. Garcia, Chemistry-a European Journal, 2011, 17, 6256-6262.

156. A. Dhakshinamoorthy, M. Alvaro and H. Garcia, Journal of Catalysis, 2012, 289, 259265.

157. A. Dhakshinamoorthy, M. Alvaro and H. Garcia, Acs Catalysis, 2011, 1, 48-53.

158. A. Corma and H. Garcia, Chemical Reviews, 2003, 103, 4307-4365.

159. R. A. Sheldon, J. D. Chen, J. Dakka and E. Neeleman, in New Developments in Selective Oxidation Ii, eds. V. C. Corberan and S. V. Bellon, Editon edn., 1994, vol. 82, pp. 515-529.

160. M. Hartmann and L. Kevan, Research on Chemical Intermediates, 2002, 28, 625-695.

161. U. Schuchardt, D. Cardoso, R. Sercheli, R. Pereira, R. S. de Cruz, M. C. Guerreiro, D. Mandelli, E. V. Spinace and E. L. Fires, Applied Catalysis a-General, 2001, 211, 1-17.

162. K. Brown, S. Zolezzi, P. Aguirre, D. Venegas-Yazigi, V. Paredes-Garcia, R. Baggio, M. A. Novak and E. Spodine, Dalton Transactions, 2009, 1422-1427.

163. M. Tonigold, Y. Lu, B. Bredenkotter, B. Rieger, S. Bahnmuller, J. Hitzbleck, G. Langstein and D. Volkmer, Angewandte Chemie-International Edition, 2009, 48, 75467550.

164. E. Q. Procopio, F. Linares, C. Montoro, V. Colombo, A. Maspero, E. Barea and J. A. R. Navarro, Angewandte Chemie-International Edition, 2010, 49, 7308-7311. 
165. K. Leus, I. Muylaert, M. Vandichel, G. B. Marin, M. Waroquier, V. Van Speybroeck and P. Van der Voort, Chemical Communications, 2010, 46, 5085-5087.

166. K. Leus, M. Vandichel, Y. Y. Liu, I. Muylaert, J. Musschoot, S. Pyl, H. Vrielinck, F. Callens, G. B. Marin, C. Detavernier, P. V. Wiper, Y. Z. Khimyak, M. Waroquier, V. Van Speybroeck and P. Van der Voort, Journal of Catalysis, 2012, 285, 196-207.

167. M. H. Xie, X. L. Yang and C. D. Wu, Chemical Communications, 2011, 47, 5521-5523.

168. K. Leus, G. Vanhaelewyn, T. Bogaerts, Y.-Y. Liu, D. Esquivel, F. Callens, G. B. Marin, V. V. Speybroeck, H. Vrielinck and P. Van Der Voort, Catal. Today, in press.

169. S. Bhattacharjee, D. A. Yang and W. S. Ahn, Chemical Communications, 2011, 47, 3637-3639.

170. R. Sen, D. Saha and S. Koner, Catalysis Letters, 2012, 142, 124-130.

171. S. H. Cho, B. Q. Ma, S. T. Nguyen, J. T. Hupp and T. E. Albrecht-Schmitt, Chemical Communications, 2006, 2563-2565.

172. N. V. Maksimchuk, M. N. Timofeeva, M. S. Melgunov, A. N. Shmakov, Y. A. Chesalov, D. N. Dybtsev, V. P. Fedin and O. A. Kholdeeva, Journal of Catalysis, 2008, 257, 315-323.

173. N. V. Maksimchuk, K. A. Kovalenko, S. S. Arzumanov, Y. A. Chesalov, M. S. Melgunov, A. G. Stepanov, V. P. Fedin and O. A. Kholdeeva, Inorganic Chemistry, 2010, 49, 2920-2930.

174. C. M. Granadeiro, A. D. S. Barbosa, P. Silva, F. A. A. Paz, V. K. Saini, J. o. Pires, B. de Castro, S. S. Balula and L. Cunha-Silva, Applied Catalysis A: General, 2013, 453, 316326.

175. J. Perles, N. Snejko, M. Iglesias and M. A. Monge, Journal of Materials Chemistry, 2009, 19, 6504-6511.

176. N. V. Maksimchuk, K. A. Kovalenko, V. P. Fedin and O. A. Kholdeeva, Advanced Synthesis \& Catalysis, 2010, 352, 2943-2948.

177. I. Y. Skobelev, A. B. Sorokin, K. A. Kovalenko, V. P. Fedin and O. A. Kholdeeva, Journal of Catalysis, 2013, 298, 61-69.

178. A. M. Balu, C. S. K. Lin, H. L. Liu, Y. W. Li, C. Vargas and R. Luque, Applied Catalysis a-General, 2013, 455, 261-266.

179. Z. Saedi, S. Tangestaninejad, M. Moghadam, V. Mirkhani and I. MohammadpoorBaltork, Catalysis Communications, 2012, 17, 18-22.

180. J. L. Long, L. M. Wang, X. F. Gao, C. H. Bai, H. F. Jiang and Y. W. Li, Chemical Communications, 2012, 48, 12109-12111.

181. N. V. Maksimchuk, K. A. Kovalenko, V. P. Fedin and O. A. Kholdeeva, Chemical Communications, 2012, 48, 6812-6814.

182. D. B. Shi, Y. W. Ren, H. F. Jiang, J. X. Lu and X. F. Cheng, Dalton Transactions, 2013, 42, 484-491.

183. X. L. Yang, M. H. Xie, C. Zou, Y. B. He, B. L. Chen, M. O'Keeffe and C. D. Wu, Journal of the American Chemical Society, 2012, 134, 10638-10645.

184. H. L. Liu, G. Z. Chen, H. F. Jiang, Y. W. Li and R. Luque, Chemsuschem, 2012, 5, 1892-1896.

185. F. Carson, S. Agrawal, M. Gustafsson, A. Bartoszewicz, F. Moraga, X. Zou and B. Martín-Matute, Chemistry - A European Journal, 2012, 18, 15337-15344.

186. H. U. Blaser and M. Studer, Applied Catalysis a-General, 1999, 189, 191-204.

187. M. S. S. Balula, I. Santos, M. M. Q. Simoes, M. Neves, J. A. S. Cavaleiro and A. M. V. Cavaleiro, Journal of Molecular Catalysis a-Chemical, 2004, 222, 159-165.

188. A. J. Stapleton, M. E. Sloan, N. J. Napper and R. C. Burns, Dalton Transactions, 2009, 9603-9615. 
189. A. K. BorgKarlson, C. R. Unelius, I. Valterova and L. A. Nilsson, Phytochemistry, 1996, 41, 1477-1483.

190. R. Anand, M. S. Hamdy, P. Gkourgkoulas, T. Maschmeyer, J. C. Jansen and U. Hanefeld, Catalysis Today, 2006, 117, 279-283.

191. S. Shylesh, C. Srilakshmi, A. P. Singh and B. G. Anderson, Microporous and Mesoporous Materials, 2007, 99, 334-344.

192. S. Opelt, S. Turk, E. Dietzsch, A. Henschel, S. Kaskel and E. Klemm, Catalysis Communications, 2008, 9, 1286-1290.

193. G. Q. Kong, S. Ou, C. Zou and C. D. Wu, Journal of the American Chemical Society, 2012, 134, 19851-19857.

194. A. E. Platero Prats, V. A. de la Peña-O'Shea, M. Iglesias, N. Snejko, Á. Monge and E. Gutiérrez-Puebla, Chemcatchem, 2010, 2, 147-149.

195. J. Hermannsdorfer, M. Friedrich, N. Miyajima, R. Q. Albuquerque, S. Kummel and R. Kempe, Angewandte Chemie-International Edition, 2012, 51, 11473-11477.

196. S. Aguado, J. Canivet and D. Farrusseng, Chemical Communications, 2010, 46, 79998001 .

197. Y. B. Huang, S. J. Liu, Z. J. Lin, W. J. Li, X. F. Li and R. Cao, Journal of Catalysis, 2012, 292, 111-117. 


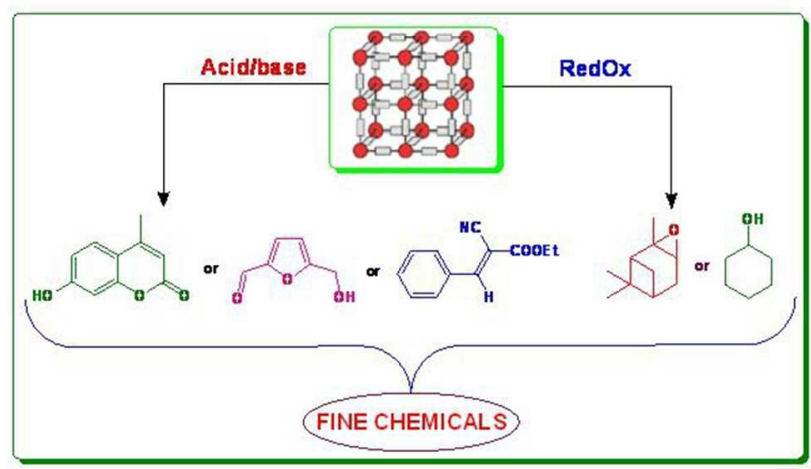

$254 \times 190 \mathrm{~mm}(96 \times 96$ DPI) 